\title{
THE ROLE OF TRUE FINITENESS IN THE \\ ADMISSIBLE RECURSIVELY ENUMERABLE \\ DEGREES
}

\author{
A Dissertation \\ Presented to the Faculty of the Graduate School \\ of Cornell University \\ in Partial Fulfillment of the Requirements for the Degree of \\ Doctor of Philosophy
}

by

Noam Greenberg

August 2004 
(c) 2004 Noam Greenberg

ALL RIGHTS RESERVED 


\title{
THE ROLE OF TRUE FINITENESS IN THE ADMISSIBLE RECURSIVELY ENUMERABLE DEGREES
}

\author{
Noam Greenberg, Ph.D.
}

Cornell University 2004

When attempting to generalize recursion theory to admissible ordinals, it may seem as if all classical priority constructions can be lifted to any admissible ordinal satisfying a sufficiently strong fragment of the replacement scheme. We show, however, that this is not always the case. In fact, there are some constructions which make an essential use of the notion of finiteness which cannot be replaced by the generalized notion of $\alpha$-finiteness. As examples we discuss both codings of models of arithmetic into the recursively enumerable degrees, and non-distributive lattice embeddings into these degrees. We show that if an admissible ordinal $\alpha$ is effectively close to $\omega$ (where this closeness can be measured by size or by cofinality) then such constructions may be performed in the $\alpha$-r.e. degrees, but otherwise they fail. The results of these constructions can be expressed in the first-order language of partially ordered sets, and so these results also show that there are natural elementary differences between the structures of $\alpha$-r.e. degrees for various classes of admissible ordinals $\alpha$. Together with coding work which shows that for some $\alpha$, the theory of the $\alpha$-r.e. degrees is complicated, we get that for every admissible ordinal $\alpha$, the $\alpha$-r.e. degrees and the classical r.e. degrees are not elementarily equivalent. 


\section{BIOGRAPHICAL SKETCH}

Noam Greenberg was born in Netanya, Israel in 1974. For his birthday he received, as a gift from a family friend, an LP of romantic and classical piano music; early on he was fascinated by Mozart's Turkish march. After graduating from high school in Ma'alot, he started playing the piano, and was very excited when his teacher introduced him to the Sonata Pathétique. He again experienced the sensation of discovery when, during his compulsory military service, he took an Open University course in Set Theory. He studied mathematics at the Hebrew University of Jerusalem and was very happy to find out that mathematical logic enabled him to better handle Bach fugues; he also received his B.Sc. in 1998.

Cornell University presented Noam with new opportunities. He joined a choir and also performed annually in the mathematics department's spring concert. From time to time he also engaged in research. He will be a post-doc at the University of Notre Dame, where he hopes to participate in a performance of the great mass in B minor (BWV 232), of which he's been daydreaming during the writing of this thesis. Thinking of his homeland and of the state of affairs of the world in general, he is particularly touched by the closing fugue: Dona Nobis Pacem. 
To my grandfather Alfred. 


\section{ACKNOWLEDGEMENTS}

Emotional support and a sustaining intellectual environment (and often both) were provided by many, only some of which I have the space here to thank. My apologies for the omissions I must make.

My family, of course, has shaped my personality. My parents, grandparents and brother introduced me to these things in life I am most attached to and most excited about. If I consider my life rich and interesting, I have them to thank for it. My parents have always made my education a priority, and I would not have done well without their support.

As other students of his have acknowledged, Richard Shore is a remarkable advisor. His mastery of the tricky art of advising includes not only mathematical knowledge and prowess but also an understanding of the strengths and weaknesses of any particular student. His patience, and generosity with his time, allowed me to both proceed without unnecessary pressure, and to become the noisy student I am today. Obvious as it is, I should say it: this thesis would not have been possible without Richard.

One sentence of sympathy from Anil Nerode has done wonders to my mathematical confidence; for that, and for the unique experience which was being his teaching assistant, he has my deep gratitude. I wish I had interacted more with Anil Nerode during my time at Cornell; he is, however, a model for me for a mathematician who will never slow down, and I hope to rectify my omission in the future.

I would also like to thank Dexter Kozen, who is a member of my committee, for his useful comments. 
At Cornell I have come to love collaborative work and to appreciate the benefits that the vibrant community of mathematical logic has provided me with. Both formal and informal interaction with students, post-docs and professors were stimulating and enjoyable. Collaboration with my colleague Antonio Montalbán proved to be fruitful. I am looking forward to interacting with him and with other Cornell graduates in the future. Antonio, Yuval, Denis, Walker, Reed, Joe - together we shall consolidate Cornell's power over the world of recursion theory.

Speaking of mathematical mafiaesque organizations, I would like to mention and thank my undergraduate colleagues from the Hebrew University: Alex, Moshez, Pezz, and Yuval. They are all dear friends. Especially, Alex's help during my last period in Jerusalem was indispensable. I now fondly think of 40 Sheshet HaYamim St. as a home.

At Cornell I was lucky to encounter nonmathematical professionals whose help was crucial during my time here. David Kooperman (CSW) helped break my fall and guided me through some tough times. His wisdom helped me become a better and happier man. My piano teacher Charlotte Greenspan is an intellectual inspiration and a friend; she helped me shatter some of the glass walls that surrounded me.

Finally, it is my pleasure to acknowledge some good friends. Ryan, Chris, Suzanne, Adi, Rebecca, Gur, Ana, Birgit and Karin have each made the world more beautiful. Thanks. 


\section{TABLE OF CONTENTS}

1 Introduction 1

1.1 The Results . . . . . . . . . . . . . . . . . . . . 7

1.1.1 Lattice Embeddings . . . . . . . . . . . . . . . . . 8

1.1.2 Effective Successor Models . . . . . . . . . . . . . . . . 9

$1.1 .3 \mathcal{R}_{\alpha}$ is Sometimes Complicated . . . . . . . . . . . . 11

2 Coding Into the R.E. Degrees 13

2.1 The Coding . . . . . . . . . . . . . . . . . . . 13

2.2 A Template for the Constructions . . . . . . . . . . . . . . 21

2.2.1 The Requirements . . . . . . . . . . . . . . . 21

2.2.2 Elements of the Constructions . . . . . . . . . . . . 23

2.2.3 Construction . . . . . . . . . . . . . . . . . . . 28

2.2 .4 Verifications . . . . . . . . . . . . . . . 32

2.3 Various Constructions . . . . . . . . . . . . . . . . . . . . . . 44

$2.3 .1 \varrho_{\alpha}=\omega \ldots \ldots \ldots \ldots \ldots \ldots$. . . . . . . . . . . . . . . . . . . . . . 44

$2.3 .2 \varrho_{\alpha}^{2}=\omega$ but $\varrho_{\alpha}>\omega \ldots \ldots \ldots \ldots . \ldots . \ldots . \ldots 46$

2.3.3 $\alpha$ is $\Sigma_{2}$-admissible, but $\operatorname{cf}_{\Sigma_{3}\left(J_{\alpha}\right)}(\alpha)=\omega \ldots \ldots . \ldots . . . . .49$

3 Coding Effective Successor Models $\quad 55$

3.1 Construction . . . . . . . . . . . . . . . 57

3.1 .1 The Machine . . . . . . . . . . . . . . . . . 58

3.1 .2 Construction . . . . . . . . . . . . . . . . 72

3.2 Verifications . . . . . . . . . . . . . . . . 75

4 A Negative Result Concerning Effective Successor Models 103

4.1 Preparation: Some Complexity Calculations . . . . . . . . . . . . 103

4.2 More on Effective Models . . . . . . . . . . . . . . . . . . . . . . . 104

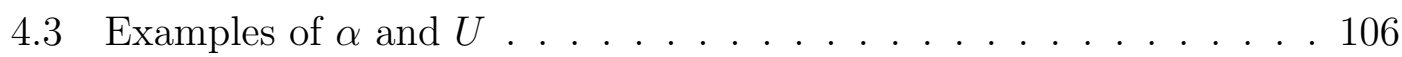

$4.3 .1 \varrho_{\alpha}^{2}=\alpha$ and $U$ is low . . . . . . . . . . . . . . . 107

4.3.2 $\alpha$ is $\Sigma_{2}$-admissible and $U$ is $\operatorname{low}_{2} \ldots \ldots \ldots$. . . . . . 107

4.3.3 $\alpha$ is $\Sigma_{3}$-admissible, $\operatorname{cf}_{\Sigma_{4}\left(J_{\alpha}\right)}(\alpha)>\omega$ and $U$ is in any r.e. degree 108

4.3.4 $\varrho_{\alpha}^{3}=\alpha, \operatorname{cf}_{\Sigma_{4}\left(J_{\alpha}\right)}(\alpha)>\omega$ and $U$ is in any r.e. degree . . . . 108

$\begin{array}{lll}5 \text { A Nonembedding Result } & 109\end{array}$

6 Embedding the 1-3-1 Lattice 112

6.1 Preparation . . . . . . . . . . . . . . . . . . 112

6.2 The Embedding . . . . . . . . . . . . . . . . . . . . . 114

6.2.1 Elements of the Construction . . . . . . . . . . . 115

6.2.2 Construction . . . . . . . . . . . . . . . . 126

6.2.3 Verifications . . . . . . . . . . . . . . . . . 127 
$\begin{array}{ll}\text { A Basics } & 136\end{array}$

$\begin{array}{lr}\text { B The Jump } & 147\end{array}$

C The Projectum 152

$\begin{array}{ll}\text { D The Admissible Collapse } & 154\end{array}$

$\begin{array}{lr}\text { E Prompt Permission } & 158\end{array}$

$\begin{array}{lr}\text { Bibliography } & 160\end{array}$ 


\section{LIST OF FIGURES}

2.1 The definition of map . . . . . . . . . . . . . . . . . 19

6.1 The $1-3-1$ lattice . . . . . . . . . . . . . . . . 112 


\section{CHAPTER 1}

\section{INTRODUCTION}

The study of recursive ordinals and hyperarithmetic sets that began with the work of Church and Kleene [CK37], Church [Chu38] and Kleene [Kle38] suggested many analogies between the $\Pi_{1}^{1}$ and hyperarithmetic sets and the recursively enumerable and recursive ones, respectively. The analogy was not perfect, however. At the basic level, for example, the range of a hyperarithmetic function on a hyperarithmetic set is always hyperarithmetic rather than an arbitrary $\Pi_{1}^{1}$ set. At a deeper level, all nonhyperarithmetic $\Pi_{1}^{1}$ sets are of the same hyperarithmetic degree. Kreisel [Kre61] studied this situation and came to the realization that while $\Pi_{1}^{1}$ is analogous to r.e., the correct analog for hyperarithmetic is not recursive but finite. This insight lead first to the development by Kreisel and Sacks [KS63, KS65] of metarecursion theory as the study of recursion theory on the recursive ordinals (those less than $\omega_{1}^{C K}$, the first nonrecursive ordinal) or, equivalently, on their notations in a $\Pi_{1}^{1}$ path through Kleene's $\mathcal{O}$. In this setting, the meta-r.e. subsets of $\omega$ are the $\Pi_{1}^{1}$ ones and the metafinite ones are hyperarithmetic.

Another approach to generalizing recursion theory to ordinals started with Takeuti's [Tak60, Tak65] development of Gödel's [G3̈9] constructible universe $L$ through a recursion theory on the class of all ordinals. These two approaches came together in the common generalization of recursion on admissible ordinals of Kripke [Kri64] and Platek [Pla65]. Here the domain of discourse is an ordinal $\alpha$ or the initial segment $L_{\alpha}$ of $L$ up to $\alpha$ for admissible $\alpha$, i.e. $L_{\alpha}$ satisfies $\Sigma_{1}$-replacement. In this vein, $\alpha$-r.e. is $\Sigma_{1}$ over $L_{\alpha}, \alpha$-recursive is then $\Delta_{1}$ over $L_{\alpha}$ while $\alpha$-finite means a member of $L_{\alpha}$. These notions coincide with those of metarecursion theory when $\alpha=\omega_{1}^{C K}$. 
We should also note that care has to be taken in the definition of " $\alpha$-recursive in", the analog of Turing reducibility. Here too, the crucial issue is that of finiteness. It no longer suffices to require that one be able to answer single membership question about $A$ in a computation from $B$ to say that $A$ is reducible to $B$. Instead one defines $\alpha$-reducible, $\leqslant_{\alpha}$, by requiring that all $\alpha$-finite sets of such questions about $A$ can be computed on the basis of $\alpha$-finitely much information about $B$.

The motivation and goals for generalizing recursion theory in this way included the hopes of elucidating the underlying nature of the notions fundamental to recursion theory and the essences of the constructions that are used to prove its most important theorems. In accordance by Kreisel's insight, a prominent role should be played by the analysis of finiteness along with recursive and recursively enumerable. Such an analysis might lead to a good axiomatic treatment or reveal approaches that would be less dependent on the specific combinatorial properties of $\omega$ exploited in these notions and constructions. In this way the study might also produce applications to both classical recursion theory and other domains (set theory, model theory, proof theory and, in hindsight, computer science) where the notions of effectiveness play many roles.

It was relatively easy to formalize the basic notions of recursion theory in these settings but also in much more general ones. Kreisel's test of a generalization worthy of investigation was the Freidberg-Muchnik theorem solving Post's problem by showing that there are incomparable r.e. degrees. As Sacks [Sac90, p. ix] puts it, this brings us from the static or syntactic realm into the dynamic one. It is in this domain that priority arguments and the deeper investigations into the notion of enumerability and relative computability were developed in classical recursion theory. First metarecursion theory (Sacks [Sac66]) and then $\alpha$-recursion theory 
(Sacks and Simpson [SS72]) passed this test.

The route to the solution to Post's problem in $\alpha$-recursion theory was the ability to make $\Sigma_{1}$-replacement suffice for arguments that in classical recursion theory seemed to naturally rely on $\Sigma_{2}$-replacement (or induction). Further investigations in $\alpha$-recursion theory indicated that many of the more complicated priority arguments of the classical subject used yet higher levels of replacement and did not generalize so readily to all admissible $\alpha$. The density theorem was successfully generalized to all admissible $\alpha$ (Shore [Sho76b]) but to this day the theorems epitomizing the basic construction of classical recursion theory have not been settled for all admissible ordinals. Almost always more admissibility suffices and at times other conditions as well. Early examples include the existence of an incomplete high $\alpha$-r.e. degree (Shore [Sho76a]) and minimal pairs (Lerman and Sacks [LS72]) for which $\Sigma_{2}$ admissibility suffices and at times something less. Eventually, an elementary difference between the r.e. degrees and the $\alpha$-r.e. degrees for some $\alpha$ was established by finding certain admissible ordinals for which, contrary to Lachlan's [Lac76] nonsplitting theorem, one can combine splitting and density for all pairs of $\alpha$-r.e. degrees (Shore [Sho78]). (That is, for certain $\alpha$ it is always possible to find, for every pair $\mathbf{a}<\mathbf{b}$ of $\alpha$-r.e. degrees, two incomparable $\alpha$-r.e. degrees $\mathbf{b}_{0}$ and $\mathbf{b}_{1}$ between $\mathbf{a}$ and $\mathbf{b}$ such that $\mathbf{b}_{0} \vee \mathbf{b}_{1}=\mathbf{b}$.) This work did indeed elucidate the role of various replacement or induction-like principles in recursion theoretic arguments and much later played a role in analyzing such arguments in reverse mathematics (e.g. Slaman and Woodin [SW89] and Mytilinaios [Myt89]). Other aspects of generalized recursion theory found applications in complexity theory (e.g. Shinoda and Slaman [SS90]). They did not however have much to say directly about the role of finiteness. Moreover, once the basic techniques are understood, all these 
constructions can be fairly easily carried out in metarecursion theory.

The crucial fact about $\omega_{1}^{C K}$ needed to carry out all these arguments is that there is a metarecursive projection of $\omega_{1}^{C K}$ into $\omega$. This allows one to arrange priority requirements in an $\omega$ list and so carry out constructions in such a way that one only ever really needs to worry about there being truly finitely many predecessors of any requirement. For example, density was proved by Driscoll [Dri68] and minimal pairs constructed by Sukonick [Suk69]. It seemed as if everything one could do in classical recursion theory could be done in metarecursion theory as well. It was in this setting that Sacks [Sac63] posed as his final question whether $\mathcal{R}_{\omega_{1}^{C K}}$, the metar.e. degrees with $\omega_{1}^{C K}$-reducibility, and $\mathcal{R}$, the r.e. ones with Turing reducibility, are elementarily equivalent. This seemed possible at the time. Indeed, at that time people still thought that there should be some nice characterization of the structure $\mathcal{R}$ that would indicate that it was simple in some way. Shoenfield's conjecture that it was $\omega$-saturated and so categorical had been disproven with the construction of a minimal pair of r.e. degrees but, nonetheless, Sacks still conjectured in [Sac63] that the theory was decidable and that the structure was isomorphic to the degrees r.e. in and above $\mathbf{d}$ for every degree $\mathbf{d}$.

Both of these conjectures turned out to be false (Harrington-Shelah [HS82], Shore [Sho82]). Indeed, these results and others showed that $\mathcal{R}$ was very complicated in various ways. Shore [Sho82] showed that it is not recursively presentable and later Harrington and Slaman and Slaman and Woodin (see Slaman [Sla91]) showed that its theory is recursively isomorphic to true arithmetic. These sorts of results changed the paradigm for understanding $\mathcal{R}$ from a hope for simplicity to an approach to its characterization by its complexity. (For more of the history and further discussion, see Shore [Sho97] and [Sho99]). Once one had this view 
of $\mathcal{R}$, it became natural to believe that the answer to Sacks' question was "no" just because it seemed that one could prove all the results of classical recursion theory in metarecursion theory. If the meta-r.e. degrees, like the r.e. ones, are as complicated as possible then $\mathcal{R}_{\omega_{1}^{C K}}$ is more complicated than $\mathcal{R}$. In this way, Odell [Ode83] established an analog of Shore [Sho82] for the meta-r.e. degrees to show that $\mathcal{R}_{\omega_{1}^{C K}}$ is not arithmetically presentable and so not isomorphic to $\mathcal{R}$. Once Harrington and Slaman and Slaman and Woodin had proven that the theory of $\mathcal{R}$ is recursively isomorphic to true arithmetic, it became "morally certain" that the two structures are not even elementarily equivalent.

Shore and Slaman, as announced in Shore [Sho97], managed to carry out enough of the relevant constructions in metarecursion theory to prove this result. The proof was fairly elaborate and required lifting several major theorems of classical recursion theory to $\omega_{1}^{C K}$. It also failed to give a full characterization of the degree of the theory of $\mathcal{R}_{\omega_{1}^{C K}}$. The expected result was that it should be recursively isomorphic to the theory of $\left\langle L_{\omega_{1}^{C K}}, \in\right\rangle$ or, equivalently, of degree $\mathcal{O}^{(\omega)}$. This result awaited further developments in classical recursion theory. Nies, Shore and Slaman [NSS98] provided a definable standard model of arithmetic in $\mathcal{R}$ and so a more direct proof that the degree of its theory is $\mathbf{0}^{(\omega)}$. In $[\mathrm{GSS}]$, the same original intuition from the $60 \mathrm{~s}$ about the similarity of $\mathcal{R}$ and $\mathcal{R}_{\omega_{1}^{C K}}$ was followed, to lift enough of Nies, Shore and Slaman [NSS98] to metarecursion theory to prove that a standard model of arithmetic with a predicate for $\mathcal{O}$ is definable in $\mathcal{R}_{\omega_{1}^{C K}}$ and so its theory, as expected, is recursively isomorphic to both that of $L_{\omega_{1}^{C K}}$ and to $\mathcal{O}^{(\omega)}$. These results thus answered Sacks's original question by providing an elementary difference between $\mathcal{R}$ and $\mathcal{R}_{\omega_{1}^{C K}}$. However, they did so by continuing along the path following the intuition that one can lift all constructions of r.e. degrees to 
$\omega_{1}^{C K}$ by using projectability to convert requirements lists to ones of length $\omega$ and to any admissible ordinal satisfying enough replacement to handle requirements in order type $\alpha$.

These illusions are hereby dispelled in this present work, whose aim is to illuminate the role of true finiteness in various classical constructions in the setting of the r.e. degrees (Lerman and Simpson [LS73] and Lerman ([Ler74]) gave such results in the context of the lattice of r.e. sets). We show here that constructions given in [NSS98] can be performed in the $\alpha$-r.e. degrees (for $\Sigma_{2}$-admissible $\alpha$ ) if and only if the cofinality of $\alpha$, as measured by some relatively effective class of functions, is $\omega$. Another line of investigation considers construction which are used to embed some nondistributive lattices into the r.e. degrees. Lachlan ([Lac72]) has shown that the 1-3-1 lattice, the one of the two basic nondistributive lattices which includes a critical triple, is embeddable in the r.e. degrees. We show that this construction uses finiteness in an essential way; it can only be performed in the $\alpha$-r.e. degrees (here $\alpha$ is any admissible ordinal) if $\alpha$ is countable in some effective sense. All of the work taken together shows that no $\mathcal{R}_{\alpha}$ is elementarily equivalent to $\mathcal{R}$.

\section{Notation and Terminology}

Rather than give a long list of definitions of the concepts used in this work and of the notation associate with them, we prefer to place each definition in its natural context as it comes up in the work. Many of these definitions make sense only together with facts concerning the objects involved; in the appendices we develop much of the theory which is needed to make most of the definitions comprehensible.

The basics of admissible recursion theory are developed in appendix A. It is there where we define our "playing ground", namely Jensen's $J_{\alpha}$ hierarchy, describe the notions of amenability and admissibility, discuss $\alpha$-recursive enumerations and 
$\alpha$-reductions, and in that context it makes sense to define nice functionals, which are not used in the standard texts of the field (Sacks [Sac90] and Chong [Cho84].) We also define there effective versions of cofinality, such as the $\Sigma_{n}$ cofinality of $\alpha$ and the recursive cofinality of a degree.

Further notions of $\alpha$-recursion theory include the jump operator (and the notion of lowness which accompanies it), described in appendix B, and the projectum $\varrho_{\alpha}^{n}$, which is discussed in appendix C.

Notions from classical recursion theory, often more algebraic in nature (such as the lattices we try to embed in the degrees, or the coding of models of arithmetic in the degrees), are defined in the body of the text as they appear.

The notation used follows modern set-theoretic standards (see, for example, Jech [Jec03]). Thus for example we use $f^{\text {" }} X$ to denote the pointwise image of $X$ under the function $f . \subset$ means inclusion, whether proper or not (when we want to stress proper inclusion we will use $\subsetneq$ ). Club means closed and unbounded (usually in the fixed admissible ordinal $\alpha$ ).

For recursion theory we use the functional notation which has become standard in recent years. the meaning of this notation in the context of admissible recursion theory is explained in appendix A. During effective constructions or enumerations we use Lachlan's notation ([Lac79]) of modifying objects and whole expressions by $[s]$ to denote they are viewed in stage $s$ of the construction or enumeration. In general notation will be similar to the one used in [Soa87, XIV s.4].

\subsection{The Results}

In this thesis we show how the proximity of an admissible ordinal $\alpha$ to $\omega$ is reflected in the structure of the $\alpha$-recursively enumerable degrees (which we denote by $\mathcal{R}_{\alpha}$ ). 


\subsubsection{Lattice Embeddings}

Theorem 1.1. There is a sentence $\psi$ (in the language of partially ordered sets) such that for every admissible ordinal $\alpha, \mathcal{R}_{\alpha}=\psi$ iff $\varrho_{\alpha}^{2}=\omega$ and $\operatorname{cf}_{\Sigma_{2}\left(J_{\alpha}\right)}(\alpha)=\omega$.

The sentence $\psi$ states the existence of an embedding of the 1-3-1 lattice (also known as $M_{5}$; see [Soa87, IX 2.7] and figure 6.1) into $\mathcal{R}_{\alpha}$ with an incomplete top. The class of ordinals $\alpha$ such that $\mathcal{R}_{\alpha} \models \psi$ is exactly the class of admissible ordinals $\alpha$ such that there is an incomplete degree $\mathbf{a} \in \mathcal{R}_{\alpha}$ such that $\operatorname{rcf}(\mathbf{a})$, the recursive cofinality of $\mathbf{a}$, is $\omega$. For the definition of recursive cofinality and $\Sigma_{2}\left(J_{\alpha}\right)$ cofinality, see the end of appendix A. For the definition of the $\Sigma_{2}$-projectum $\varrho_{\alpha}^{2}$ see appendix C.

In chapter 5 we show (theorem 5.4) that if $\alpha$ is admissible and $\operatorname{rcf}(\mathbf{a})>\omega$ then the 1-3-1 lattice cannot be embedded below $\mathbf{a}$; in fact, there is no weak critical triple below a (see definition in chapter 5). In chapter 6 (theorem 6.1) we show that if $\mathbf{a} \in \mathcal{R}_{\alpha}$ is collapsible then we can embed the 1-3-1 lattice below $\mathbf{a}$. In appendix D (where the notion of a collapsible degree is defined) we quote Shore ([Sho76b]) and see that if $\mathbf{a} \in \mathcal{R}_{\alpha}$ is incomplete and $\operatorname{rcf}(\mathbf{a})=\omega$ then $\mathbf{a}$ is collapsible.

The following theorem is also an immediate consequence of these results:

Theorem 1.2. Let $\alpha$ be admissible. The following are equivalent for an incomplete degree $\mathbf{a} \in \mathcal{R}_{\alpha}$ :

1. There is a weak critical triple in $\mathcal{R}_{\alpha}(\leqslant \mathbf{a})$.

2. There is an embedding of the 1-3-1 lattice into $\mathcal{R}_{\alpha}(\leqslant \mathbf{a})$.

3. $\operatorname{rcf}(\mathbf{a})=\omega$. 
This gives us two formulas $\varphi_{0}$ and $\varphi_{1}$ (which are not equivalent in the theory of partial orderings or in the theory of upper semi-lattices) such that for every admissible $\alpha$,

$$
\varphi_{0}\left(\mathcal{R}_{\alpha}\right)=\varphi_{1}\left(\mathcal{R}_{\alpha}\right)=\left\{\mathbf{a} \in \mathcal{R}_{\alpha}: \mathbf{a}<\mathbf{0}^{\prime} \& \operatorname{rcf}(\mathbf{a})=\omega\right\}
$$

\subsubsection{Effective Successor Models}

In chapter 3 we show that if $\alpha$ is $\Sigma_{2}$-admissible and $\operatorname{cf}_{\Sigma_{3}\left(J_{\alpha}\right)}=\omega$ then one can embed an effective successor copy of the standard model of arithmetic into $\mathcal{R}_{\alpha}$ below any promptly permitting degree $\mathbf{u}$, such that below $\mathbf{u}$ there is no least upper bound for the elements of the model. [See the definition of our coding of models of arithmetic in chapter 2 and that of an effective successor model in chapter 3. Prompt permission is discussed in appendix E.] In chapter 4 we show that for various classes of $\alpha$, including the class of $\Sigma_{2}$-admissible ordinals such that $\operatorname{cf}_{\Sigma_{3}\left(J_{\alpha}\right)}(\alpha)>\omega$, there can be no such embedding below any low 2 degree $\mathbf{u}$.

We give some details. Let $\chi_{\text {effective }}(\overline{\mathbf{p}}, \overline{\mathbf{e}})$ be the formula stating that $\overline{\mathbf{p}}$ codes an effective successor model of arithmetic, witnessed by $\mathbf{\mathbf { e }}$; see chapter 3 [We denote the model coded by $\overline{\mathbf{p}}$ by $\left.M_{\overline{\mathbf{p}}}\right]$. Theorem 3.1 states that if $\alpha$ is $\Sigma_{2}$-admissible, $\operatorname{cf}_{\Sigma_{3}\left(J_{\alpha}\right)}=\omega$ and $\mathbf{u}$ is a promptly permitting degree then there are some $\overline{\mathbf{p}}, \overline{\mathbf{e}} \leqslant \mathbf{u}$ satisfying $\chi_{\text {effective }}$ such that $M_{\overline{\mathbf{p}}}$ is standard (i.e. is isomorphic to the standard model of arithmetic), and such that there is an exact pair $\mathbf{c}_{0}, \mathbf{c}_{1} \leqslant \mathbf{u}$ for the elements of $M_{\overline{\mathbf{p}}}$. The existence of such a pair implies that in $\mathcal{R}_{\alpha}(\leqslant \mathbf{u})$, the degrees below $\mathbf{u}, M_{\overline{\mathbf{p}}}$ has no least upper bound. Also, because $M_{\overline{\mathbf{p}}}$ is standard, $M_{\overline{\mathbf{p}}}$ is the only nontrivial initial segment of $M_{\overline{\mathbf{p}}}$ closed under the successor operation.

However, in chapter 4 we show (theorem 4.4) that there is a formula $\theta$ such that if $\alpha$ is $\Sigma_{2}$-admissible, $\operatorname{cf}_{\Sigma_{3}\left(J_{\alpha}\right)}(\alpha)>\omega$ and $\mathbf{u}$ is $\operatorname{low}_{2}$, then for all $\overline{\mathbf{p}}, \overline{\mathbf{e}} \leqslant \mathbf{u}$ 
which satisfy $\chi_{\text {effective }}$ there is some $\mathbf{c} \leqslant \mathbf{u}$ which is the least upper bound for the standard part of $M_{\overline{\mathbf{p}}}$ in the degrees below $\mathbf{u}$; moreover, that standard part is definable as $\theta\left(\mathcal{R}_{\alpha}, \mathbf{c}, \overline{\mathbf{p}}\right)$.

We can thus let the formula $\phi_{0}(y, \mathbf{c}, \overline{\mathbf{p}}, \overline{\mathbf{e}})$ state that $\mathbf{c}, \overline{\mathbf{p}}, \overline{\mathbf{e}}<y$, and that $\chi_{\text {effective }}(\overline{\mathbf{p}}, \overline{\mathbf{e}})$ holds, that $\theta\left(\mathcal{R}_{\alpha}, \mathbf{c}, \overline{\mathbf{p}}\right)$ is a nontrivial initial segment of $M_{\overline{\mathbf{p}}}$ closed under the successor operation, and that $\mathbf{c}$ is the least upper bound for $\theta\left(\mathcal{R}_{\alpha}, \mathbf{c}, \overline{\mathbf{p}}\right)$ in the degrees below $y$. Let $\phi(y)$ state the existence of some $\overline{\mathbf{p}}, \overline{\mathbf{e}}$ below $y$ such that $\chi_{\text {effective }}(\overline{\mathbf{p}}, \overline{\mathbf{e}})$ holds but for no $\mathbf{c}$ below $y$ does $\phi_{0}(y, \mathbf{c}, \overline{\mathbf{p}}, \overline{\mathbf{e}})$ hold. We thus argued that for a $\Sigma_{2}$-admissible ordinal $\alpha$ such that $\operatorname{cf}_{\Sigma_{3}\left(J_{\alpha}\right)}(\alpha)=\omega, \phi(\mathbf{u})$ holds (in $\mathcal{R}_{\alpha}$ ) for all $\mathbf{u}$ which permit promptly; and that if $\alpha$ is $\Sigma_{2}$-admissible such that $\operatorname{cf}_{\Sigma_{3}\left(J_{\alpha}\right)}(\alpha)>\omega$, then $\neg \phi(\mathbf{u})$ holds for all $\mathbf{u}$ which are $\operatorname{low}_{2}$.

Let $X$ be an additional unary predicate. Let PS denote the collection of degrees which permit promptly; let $\mathbf{L}_{1}$ and $\mathbf{L}_{2}$ denote the classes of low and low 2 degrees respectively. Now there is always a low promptly permitting degree. Together with the results mentioned, we get the following:

Theorem 1.3. Let $\alpha$ be a $\Sigma_{2}$-admissible ordinal.

1. $\left(\mathcal{R}_{\alpha}, \mathbf{P S}\right) \models \forall y \in X \phi(y)$ iff $\operatorname{cf}_{\Sigma_{3}\left(J_{\alpha}\right)}(\alpha)=\omega$.

2. $\left(\mathcal{R}_{\alpha}, \mathbf{L}_{1}\right) \models \exists y \in X \phi(y)$ iff $\left(\mathcal{R}_{\alpha}, \mathbf{L}_{2}\right) \models \exists y \in X \phi(y)$ iff $\operatorname{cf}_{\Sigma_{3}\left(J_{\alpha}\right)}(\alpha)=\omega$.

One would like of course to improve this by eliminating the extra unary predicate; one would think that the most likely candidate is the class of promptly permitting degrees, which is definable in $\mathcal{R}_{\omega}$. The classical proof can be carried out if, for example, $\varrho_{\alpha}^{2}=\omega$, but fails miserably in other cases, and we in fact suspect that in some cases there may be a noncapping degree (i.e. a degree which is not half of a minimal pair) which is not promptly promptly permitting. It follows, 
though (as no degree which permits promptly can be half of a minimal pair), that if we let $X$ state that $y$ is noncappable then for every $\Sigma_{2}$-admissible ordinal $\alpha$, if $\operatorname{cf}_{\Sigma_{3}\left(J_{\alpha}\right)}(\alpha)>\omega$ then $\mathcal{R}_{\alpha}=\exists y \in X \phi(y)$ and if $\varrho_{\alpha}^{2}=\omega$ then $\mathcal{R}_{\alpha}=\neg \exists y \in X \phi(y)$.

If we are willing to go one level higher to the $\Sigma_{3}$ level, then we get the following (this is also a consequence of theorem 4.4 and the examples given after that theorem).

Theorem 1.4. If $\operatorname{cf}_{\Sigma_{4}\left(J_{\alpha}\right)}(\alpha)>\omega$ and either $\alpha$ is $\Sigma_{3}$-admissible or $\varrho_{\alpha}^{3}=\alpha$, then $\mathcal{R}_{\alpha} \models \neg \exists y \phi(y)$.

This is an elementary difference between such $\alpha$ s and $\Sigma_{2}$-admissible $\alpha$ s such that $\operatorname{cf}_{\Sigma_{3}\left(J_{\alpha}\right)}(\alpha)=\omega$

\subsection{3 $\mathcal{R}_{\alpha}$ is Sometimes Complicated}

In chapter 2 we show that for some $\alpha$, the theory of $\mathcal{R}_{\alpha}$ is complicated, in terms of its complexity. We show:

Theorem 1.5. Let $\alpha$ be an admissible ordinal. If $\varrho_{\alpha}^{2}=\omega$ or if $\alpha$ is $\Sigma_{2}$-admissible and $\operatorname{cf}_{\Sigma_{3}\left(J_{\alpha}\right)}(\alpha)=\omega$ then $\mathcal{O}^{(\omega)} \leqslant_{1} \operatorname{Th}\left(\mathcal{R}_{\alpha}\right)$ (where $\mathcal{O}$ is Kleene's complete $\Pi_{1}^{1}$ set).

The method of proof is detailed in section 2.1. The general idea follows the constructions of [NSS98]. We show that there is a way to code structures in the language of arithmetic in a uniform way using parameters; and then show that there is a nonempty correctness condition on the parameters which is first order and which ensures that the model coded is standard. Further, we show that there is a uniform way to define the isomorphism between any two such models, so that identifying all copies we get a parameterless interpretation of $\mathbb{N}$ in $\mathcal{R}_{\alpha}$. Further, 
we show that we can code $\mathcal{O}$ in such a way and get a parameterless definition of $\mathcal{O}$ (in the coded model); this implies the theorem.

Let $\psi$ be the sentence given by theorem 1.1. $\psi$ holds in the classical r.e. degrees. For $\mathcal{R}_{\alpha}$ we get a dichotomy: if $\varrho_{\alpha}^{2}>\omega$ then $\psi$ fails in $\mathcal{R}_{\alpha}$; and if $\varrho_{\alpha}^{2}=\omega$ then $\operatorname{Th}\left(\mathcal{R}_{\alpha}\right)$ is complicated (in particular, it is not hyperarithmetic, whereas $\operatorname{Th}\left(\mathcal{R}_{\omega}\right)$ which has complexity $\mathbf{0}^{(\omega)}$ lies low in the hyperarithmetic hierarchy.) We get the following.

Theorem 1.6. For every admissible ordinal $\alpha, \mathcal{R}_{\alpha}$ and $\mathcal{R}_{\omega}$ are not elementarily equivalent. 


\section{CHAPTER 2}

\section{CODING INTO THE R.E. DEGREES}

In this chapter we use the machinery of SW-sets developed in [NSS98] and apply it to the $\alpha$-r.e. degrees for various admissible ordinals $\alpha$. Using it we code models of arithmetic (with an extra unary predicate) into the $\alpha$-r.e. degrees, and show how (using the machinery of comparison maps) we can pick out the standard models in which the unary predicate is interpreted as a relatively complicated set (the main example is Kleene's $\mathcal{O}$ ). For these $\alpha$ s we immediately get that $\operatorname{Th}\left(\mathcal{R}_{\alpha}\right)$ is at least as complicated as the set coded (and in particular is not hyperarithmetic and not equal to $\left.\operatorname{Th}\left(\mathcal{R}_{\omega}\right)\right)$.

\subsection{The Coding}

Fix an admissible ordinal $\alpha$ and work in the $\alpha$-r.e. degrees, $\mathcal{R}_{\alpha}$. We repeat the coding machinery which is developed in [NSS98].

Definition. The $S W$ set defined by a quadruple of parameters $\overline{\mathbf{p}}=(\mathbf{p}, \mathbf{q}, \mathbf{r}, \mathbf{l})$ (denoted by $G_{\overline{\mathbf{p}}}$ ) is the collection of $\mathbf{g}<\mathbf{r}$ which are minimal solutions of the inequality $\mathbf{g} \vee \mathbf{p} \geqslant \mathbf{q}$. For $\mathbf{g}_{0}, \mathbf{g}_{1} \in G_{\overline{\mathbf{p}}}$, we let $\mathbf{g}_{0} \leqslant \overline{\mathbf{p}} \mathbf{g}_{1}$ if $\mathbf{g}_{0} \leqslant \mathbf{g}_{1} \vee \mathbf{l}$.

Remark 2.1. $G_{\overline{\mathbf{p}}}$ may be empty, finite or infinite, but if not empty is always an antichain of degrees. $\leqslant_{\overline{\mathbf{p}}}$ is always a pre-partial ordering on $G_{\overline{\mathbf{p}}}$.

Next, we decode structures for the language of arithmetic from the partial ordering $\leqslant_{\overline{\mathbf{p}}}$. Let $\overline{\mathbf{p}}$ be a quadruple of parameters. In what follows we define several relations on $G_{\overline{\mathbf{p}}}$ and describe conditions on the sets and relations defined. All of these conditions together are taken as a correctness condition $\chi_{\mathbf{s w}}(\overline{\mathbf{p}})$ on the 
parameters (and indeed we note that all conditions are expressible in a first order way in the language of partial orderings, so that $\chi_{\mathrm{sw}}\left(\mathcal{R}_{\alpha}\right)$ is definable in $\left(\mathcal{R}_{\alpha}, \leqslant \alpha\right)$.)

1. $G_{\overline{\mathbf{p}}}$ is nonempty and $\leqslant_{\overline{\mathbf{p}}}$ is a partial ordering on $G_{\overline{\mathbf{p}}}$.

2. $M_{\overline{\mathbf{p}}}$ is defined to be the collection of $\leqslant_{\overline{\mathbf{p}}}$-minimal elements. $M_{\overline{\mathbf{p}}}$ is nonempty.

3. $\operatorname{pair}_{\overline{\mathbf{p}}}(\mathbf{a}, \mathbf{b}, \mathbf{c})$ holds if $\mathbf{a}, \mathbf{b} \in M_{\overline{\mathbf{p}}}$ and there is a 2 -chain (in $\leqslant_{\overline{\mathbf{p}}}$ ) between $\mathbf{a}$ and $\mathbf{c}$ and a 3-chain between $\mathbf{b}$ and $\mathbf{c}$. pair $\mathbf{p}$ defines a total function from $M_{\overline{\mathbf{p}}}^{2}$ to $G_{\overline{\mathbf{p}}}$ and so we write $\mathbf{c}=\operatorname{pair}_{\overline{\mathbf{p}}}(\mathbf{a}, \mathbf{b})$.

4. $+_{\overline{\mathbf{p}}}(\mathbf{a}, \mathbf{b}, \mathbf{c})$ holds if $\mathbf{a}, \mathbf{b}, \mathbf{c} \in M_{\overline{\mathbf{p}}}$ and there is a 4-chain in $\leqslant_{\overline{\mathbf{p}}}$ between $\mathbf{c}$ and $\operatorname{pair}_{\overline{\mathbf{p}}}(\mathbf{a}, \mathbf{b}) .+_{\overline{\mathbf{p}}}$ defines a total function on $M_{\overline{\mathbf{p}}}^{2}$; we write $\mathbf{c}=\mathbf{a}+_{\overline{\mathbf{p}}} \mathbf{b}$.

5. $\times_{\overline{\mathbf{p}}}(\mathbf{a}, \mathbf{b}, \mathbf{c})$ holds if $\mathbf{a}, \mathbf{b}, \mathbf{c} \in M_{\overline{\mathbf{p}}}$ and there is a 5 -chain in $\leqslant_{\overline{\mathbf{p}}}$ between $\mathbf{c}$ and $\operatorname{pair}_{\overline{\mathbf{p}}}(\mathbf{a}, \mathbf{b}) . \times_{\overline{\mathbf{p}}}$ defines a total function on $M_{\overline{\mathbf{p}}}^{2}$; we write $\mathbf{c}=\mathbf{a} \times_{\overline{\mathbf{p}}} \mathbf{b}$.

6. $\left(M_{\overline{\mathbf{p}}},+_{\overline{\mathbf{p}}}, \times_{\overline{\mathbf{p}}}\right)$ satisfies some sufficiently rich finite fragment of arithmetic (say Robinson arithmetic); the important fact is that $M_{\overline{\mathbf{p}}}$ always has a standard initial segment. As is a usual practice, if $\varphi$ is a formula in first order arithmetic then we let $\varphi\left(M_{\overline{\mathbf{p}}}\right)$ or $\varphi^{M_{\overline{\mathbf{p}}}}$ denote its interpretation in $\left(M_{\overline{\mathbf{p}}},+_{\overline{\mathbf{p}}}, \times_{\overline{\mathbf{p}}}\right)$. We thus get $n^{M_{\overline{\mathbf{p}}}}$ for each $n<\omega, \leqslant^{M_{\overline{\mathbf{p}}}}$ (not to be confused with $\leqslant_{\overline{\mathbf{p}}}$ ), etc.

7. We let $X_{\overline{\mathbf{p}}}$ be the collection of $\mathbf{a} \in M_{\overline{\mathbf{p}}}$ such that there is a 6 -chain in $\leqslant_{\overline{\mathbf{p}}}$ above $\mathbf{a}$.

Remark 2.2. Let $X \subset \omega$. Then we can easily produce a partial ordering $\preccurlyeq x$ which codes the standard model of arithmetic with extra predicate $X$. That is, there is some partial ordering $\preccurlyeq x$ on $\omega$ (which is effectively computed from $X$ ) such that for all $\overline{\mathbf{p}}$, if $\chi_{\mathrm{SW}}(\overline{\mathbf{p}})$ holds and $\left(G_{\overline{\mathbf{p}}}, \leqslant_{\overline{\mathbf{p}}}\right) \cong(\omega, \preccurlyeq X)$ then $\left(M_{\overline{\mathbf{p}}},+_{\overline{\mathbf{p}}}, \times_{\overline{\mathbf{p}}}\right)$ is isomorphic to the standard model of arithmetic and $X=\left\{n<\omega: n^{M_{\overline{\mathbf{p}}}} \in X_{\overline{\mathbf{p}}}\right\}$. 
We proceed along with [NSS98] to construct comparison maps. We first spell out the necessary recursion theoretic facts that are used.

For the rest of the section, let $\alpha$ be an admissible ordinal which satisfies one of the following:

- $\varrho_{\alpha}^{2}=\omega$.

- $\alpha$ is $\Sigma_{2}$-admissible and $\operatorname{cf}_{\Sigma_{3}\left(J_{\alpha}\right)}(\alpha)=\omega$.

See appendix A for the definitions of $\Sigma_{2}$-admissibility and the $\Sigma_{n}$-cofinality, and appendix $\mathrm{C}$ for the definition of the $\Sigma_{2}$-projectum $\varrho_{\alpha}^{2}$.

For such $\alpha$, we will prove the following theorems:

Theorem 2.3. Let $\preccurlyeq$ be an $\alpha$-recursive partial ordering on $\omega$, and let $\mathbf{a}>\mathbf{0}$ be a nonzero $\alpha$-r.e degree. Then there is a tuple $\overline{\mathbf{p}}=(\mathbf{p}, \mathbf{q}, \mathbf{r}, \mathbf{l})$ such that $\mathbf{r}$ is low, $\mathbf{r} \leqslant \mathbf{a}$, and such that $(\omega, \preccurlyeq) \cong\left(G_{\overline{\mathbf{p}}}, \leqslant_{\overline{\mathbf{p}}}\right)$.

If $\mathbf{r}$ is low we also say that the SW set $G_{\overline{\mathbf{p}}}$ is low. If $\mathbf{r} \leqslant \mathbf{a}$ then we say that the SW set $G_{\overline{\mathbf{p}}}$ is coded below $\mathbf{a}$. If $(\omega, \preccurlyeq) \cong\left(G_{\overline{\mathbf{p}}}, \aleph_{\overline{\mathbf{p}}}\right)$ we say that $G_{\overline{\mathbf{p}}}$ codes $\preccurlyeq$.

Theorem 2.4. Let $\preccurlyeq$ be an $\alpha$-recursive partial ordering on $\omega$ and let $H$ be an $\alpha$ recursive set of $\preccurlyeq$-minimal elements. Also let $\left\langle\mathbf{u}_{i}\right\rangle_{i \in H}$ be a sequence of uniformly $\alpha$ r.e. degrees, and $\left\langle\mathbf{v}_{i, j}\right\rangle_{i \in H, j<\omega}$ be an array of uniformly $\alpha$-r.e., uniformly low degrees such that for all $i \in H$ and $j<\omega, \mathbf{u}_{i} \nless \mathbf{v}_{i, j}$. Then there is a tuple $\overline{\mathbf{p}}=(\mathbf{p}, \mathbf{q}, \mathbf{r}, \mathbf{l})$ such that $\mathbf{r}$ is low and such that we can enumerate $G_{\overline{\mathbf{p}}}=\left\{\mathbf{g}_{i}: i<\omega\right\}$, such that $(\omega, \preccurlyeq) \cong\left(G_{\overline{\mathbf{p}}}, \leqslant_{\overline{\mathbf{p}}}\right)$ by the isomorphism $i \rightarrow \mathbf{g}_{i}$ and such that for $i \in H$ and $j<\omega$, $\mathbf{g}_{i} \leqslant \mathbf{u}_{i}$ and $\mathbf{g}_{i} \not \mathbf{v}_{i, j}$.

In the above theorems and in the following arguments we use the notion of lowness, which is discussed in appendix B. 
We first show that there is a uniform way to define comparison maps from models $M_{\overline{\mathbf{p}}_{0}} \rightarrow M_{\overline{\mathbf{p}}_{1}}$ if both models are low and $M_{\overline{\mathbf{p}}_{0}}$ is standard.

Definition. Let $\overline{\mathbf{p}}_{0}, \overline{\mathbf{p}}_{1}$ be tuples such that $\chi_{\mathrm{Sw}}\left(\overline{\mathbf{p}}_{i}\right)$ holds for $i<2$. A function $h$ is a comparison map from $M_{\overline{\mathbf{p}}_{0}}$ to $M_{\overline{\mathbf{p}}_{1}}$ if it is a 1-1 function from an initial segment of $M_{\overline{\mathbf{p}}_{0}}$ onto an initial segment of $M_{\overline{\mathbf{p}}_{1}}$ preserving the least element (i.e. $h\left(0^{M_{\overline{\mathbf{p}}_{0}}}\right)=0^{M_{\overline{\mathbf{p}}_{1}}}$ and the successor relation.

Lemma 2.5. There is a formula $\operatorname{map}_{\text {low }}(x, y, \bar{w}, \bar{z})$ such that for all tuples $\overline{\mathbf{p}}_{0}, \overline{\mathbf{p}}_{1}$ satisfying $\chi_{\mathrm{Sw}}$ such that $\mathbf{r}_{0}, \mathbf{r}_{1}$ are low and $M_{\overline{\mathbf{p}}_{0}}$ is isomorphic to the standard model of arithmetic, $\operatorname{map}_{\text {low }}\left(x, y, \overline{\mathbf{p}}_{0}, \overline{\mathbf{p}}_{1}\right)$ defines a comparison map from $M_{\overline{\mathbf{p}}_{0}}$ to $M_{\overline{\mathbf{p}}_{1}}$ which is total on $M_{\overline{\mathbf{p}}_{0}}$.

If $\chi_{\mathrm{sw}}(\overline{\mathbf{p}})$ holds and $M_{\overline{\mathbf{p}}}$ is isomorphic to the standard model of arithmetic then we say that $M_{\overline{\mathbf{p}}}$ is standard. If $\chi(\overline{\mathbf{p}})$ holds and $G_{\overline{\mathbf{p}}}$ is low then we also say that $M_{\overline{\mathbf{p}}}$ is low; if $G_{\overline{\mathbf{p}}}$ is coded below $\mathbf{a}$ then we also say that $M_{\overline{\mathbf{p}}}$ is coded below $\mathbf{a}$.

Proof. The formula map low $_{\text {is }}$ isefined as follows. Let $\psi(x, y, \bar{u}, \bar{v}, \bar{z}, t)$ state that $\chi_{\mathrm{Sw}}$ holds for $\bar{u}, \bar{v}$ and $\bar{z}$; that $x \in M_{\bar{u}}, y \in M_{\bar{v}}$ and $t \in M_{\bar{z}}$; and that there is some $s \in M_{\bar{z}}$ such that $s<^{M_{\bar{z}}} t$ and such that

1. $x$ is the least element of $M_{\bar{u}}$ (according to $<^{M_{\bar{u}}}$ ) such that $s \leqslant x$; and

2. $y$ is the least element of $M_{\bar{v}}$ (according to $\left.<{ }^{M_{\bar{v}}}\right)$ such that $\left(s+{ }_{\bar{z}} t\right) \leqslant x$.

We let $\operatorname{map}_{\text {low }}(x, y, \bar{u}, \bar{w})$ state the existence of some $\bar{z}$ and $t$ such that

$$
\psi(-,-, \bar{u}, \bar{v}, \bar{z}, t)
$$

defines a comparison map from $M_{\bar{u}}$ to $M_{\bar{v}}$, and such that this map takes $x$ to $y$. 
Now it is clear that if $M_{\overline{\mathbf{p}}_{0}}$ is standard then for any $\overline{\mathbf{p}}_{1}$ (satisfying $\chi_{\mathrm{Sw}}$ of course), $\operatorname{map}_{\text {low }}\left(x, y, \overline{\mathbf{p}}_{0}, \overline{\mathbf{p}}_{1}\right)$ defines a comparison map from $M_{\overline{\mathbf{p}}_{0}}$ to $M_{\overline{\mathbf{p}}_{1}}$. We wish to show that if in addition both $M_{\overline{\mathbf{p}}_{0}}$ and $M_{\overline{\mathbf{p}}_{1}}$ are low then $\operatorname{map}_{\text {low }}\left(x, y, \overline{\mathbf{p}}_{0}, \overline{\mathbf{p}}_{1}\right)$ is total. Fix such $\overline{\mathbf{p}}_{0}$ and $\overline{\mathbf{p}}_{1}$. It suffices to show, for every $n<\omega$, the existence of some $\overline{\mathbf{p}}$ such that $\chi_{\mathrm{sw}}(\overline{\mathbf{p}})$ holds and such that the further following property holds:

- For all $i, j<n, i^{M_{\overline{\mathbf{p}}}} \leqslant j^{M_{\overline{\mathbf{p}}_{0}}}$ iff $i=j$ and $(i+n)^{M_{\overline{\mathbf{p}}}} \leqslant j^{M_{\overline{\mathbf{p}}_{1}}}$ iff $i=j$.

The existence of such $\overline{\mathbf{p}}$ (we can even require that $M_{\overline{\mathbf{p}}}$ be standard) follows from theorem 2.4 by making the following substitutions. We let $\preccurlyeq$ be the usual partial ordering which codes the standard model of arithmetic (see remark 2.2). For $m<\omega$ let $h_{m}$ be the element of $\preccurlyeq$ coding $m$ and let $H=\left\{h_{m}: m<2 n\right\}$. For $m<n$ we let $\mathbf{u}_{h_{m}}=m^{M_{\overline{\mathbf{p}}_{0}}}$ and $\mathbf{u}_{h_{m+n}}=m^{M_{\overline{\mathbf{p}}_{1}}}$. For $i, j<n$ such that $i \neq j$, let $\mathbf{v}_{h_{m}, j}=j^{M_{\overline{\mathbf{p}}_{0}}}$ and $\mathbf{v}_{h_{m+n}, j}=j^{M_{\overline{\mathbf{p}}_{0}}}$; for all other $j<\omega$, let $\mathbf{v}_{h_{i}, j}=\mathbf{v}_{h_{i+n}, j}=\mathbf{0}$. The condition $\mathbf{u}_{i} \nless \mathbf{v}_{i, j}$ is fulfilled because $M_{\overline{\mathbf{p}}_{0}}$ and $M_{\overline{\mathbf{p}}_{1}}$ are antichains of $\alpha$-r.e. degrees. Finally, the (uniform) lowness condition follows from the lowness of $M_{\overline{\mathbf{p}}_{0}}$ and $M_{\overline{\mathbf{p}}_{1}}$.

Fix $n<\omega$ and get $\overline{\mathbf{p}}$ as described.

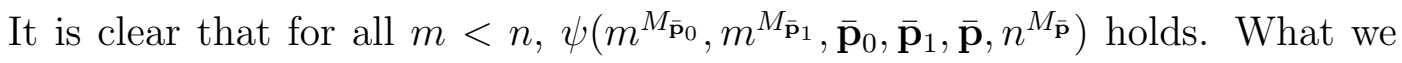
need is to show that $\psi\left(x, y, \overline{\mathbf{p}}_{0}, \overline{\mathbf{p}}_{1}, \overline{\mathbf{p}}, n^{M_{\overline{\mathbf{p}}}}\right)$ defines a comparison map from $M_{\overline{\mathbf{p}}_{0}}$ to $M_{\overline{\mathbf{p}}_{1}}$. But in fact it is not difficult to see that for all $x$ and $y, \psi\left(x, y, \overline{\mathbf{p}}_{0}, \overline{\mathbf{p}}_{1}, \overline{\mathbf{p}}, n^{M_{\overline{\mathbf{p}}}}\right)$ holds iff for some $m<n$ we have $x=m^{M_{\overline{\mathbf{p}}_{0}}}$ and $y=m^{M_{\overline{\mathbf{p}}_{1}}}$. For suppose that $\psi\left(x, y, \overline{\mathbf{p}}_{0}, \overline{\mathbf{p}}_{1}, \overline{\mathbf{p}}, n^{M_{\overline{\mathbf{p}}}}\right)$ holds; let $s \in M_{\overline{\mathbf{p}}}$ witnesses this fact. $\psi$ implies that $s<{ }^{M_{\overline{\mathbf{p}}}}$ $n^{M_{\overline{\mathbf{p}}}}$ and so $s=m^{M_{\overline{\mathbf{p}}}}$ for some $m<n$. Then $s \leqslant m^{M_{\overline{\mathbf{p}}_{0}}, x \text { and both are minimal }}$ with respect to that property, so $x=m^{M_{\overline{\mathbf{p}}_{0}}} ;$ similarly, $y=m^{M_{\overline{\mathbf{p}}_{1}}}$.

We now get a correctness condition strengthening $\chi_{\mathbf{s w}}$ which implies that the 
model coded is standard, and we get comparison maps between the models $M_{\overline{\mathbf{p}}}$ satisfying this condition.

Theorem 2.6. There is a formula $\chi_{\text {standard }}(\overline{\mathbf{p}})$ (implying $\chi_{\mathrm{SW}}(\overline{\mathbf{p}})$ ) such that for all $\overline{\mathbf{p}}$ satisfying $\chi_{\text {standard }}, M_{\overline{\mathbf{p}}}$ is standard. There is a formula map such that for every $\overline{\mathbf{p}}_{0}, \overline{\mathbf{p}}_{1}$ satisfying $\chi_{\text {standard }}, \operatorname{map}\left(x, y, \overline{\mathbf{p}}_{0}, \overline{\mathbf{p}}_{1}\right)$ defines the isomorphism between $M_{\overline{\mathbf{p}}_{0}}$ and $M_{\overline{\mathbf{p}}_{1}}$. Further, if $X \subset \omega$ is $\alpha$-recursive, then there is some $\overline{\mathbf{p}}$ such that $\chi_{\text {standard }}(\overline{\mathbf{p}})$ holds and $M_{\overline{\mathbf{p}}}$ codes $X$.

Proof. $\chi_{\text {standard }}(\overline{\mathbf{p}})$ states that $\chi_{\mathrm{sw}}(\overline{\mathbf{p}})$ holds and that for every other tuple $\overline{\mathbf{p}}^{\prime}=$ $\left(\mathbf{p}^{\prime}, \mathbf{q}^{\prime}, \mathbf{r}^{\prime}, \mathbf{l}^{\prime}\right)$ satisfying $\chi_{\mathrm{sw}}$ such that $\mathbf{r}^{\prime} \leqslant \mathbf{r}, \operatorname{map}_{\text {low }}\left(x, y, \overline{\mathbf{p}}, \overline{\mathbf{p}}^{\prime}\right)$ defines a total map on $M_{\overline{\mathbf{p}}}$.

Suppose that $\chi_{\text {standard }}(\overline{\mathbf{p}})$ holds. By theorem 2.3, we know that there is some $\overline{\mathbf{p}}^{\prime}$ such that $\mathbf{r}^{\prime} \leqslant \mathbf{r}$ and such that $M_{\overline{\mathbf{p}}^{\prime}}$ is standard. Totality of the comparison map from $M_{\overline{\mathbf{p}}}$ into $M_{\overline{\mathbf{p}}^{\prime}}$ implies that $M_{\overline{\mathbf{p}}}$ is standard as well.

To define map, we note that in the proof of lemma 2.5, we say that whenever $M_{\overline{\mathbf{p}}}$ is standard and $M_{\overline{\mathbf{p}}^{\prime}}$ is any other coded model, map low $\left(x, y, \overline{\mathbf{p}}, \overline{\mathbf{p}}^{\prime}\right)$ defines a comparison map from $M_{\overline{\mathbf{p}}}$ to $M_{\overline{\mathbf{p}}^{\prime}}$, not necessarily total. For such $\overline{\mathbf{p}}$ and $\overline{\mathbf{p}}^{\prime}$, let $h_{\overline{\mathbf{p}}, \overline{\mathbf{p}}^{\prime}}$ denote this comparison map.

Now let $\overline{\mathbf{p}}_{0}, \overline{\mathbf{p}}_{3}$ be two tuples satisfying $\chi_{\text {standard }}$. We let $\operatorname{map}\left(x, y, \overline{\mathbf{p}}_{0}, \overline{\mathbf{p}}_{3}\right)$ state that there are $\overline{\mathbf{p}}_{1}, \overline{\mathbf{p}}_{2}$, also satisfying $\chi_{\text {standard }}$, such that

$$
y=h_{\overline{\mathbf{p}}_{3}, \overline{\mathbf{p}}_{2}}^{-1} \circ h_{\overline{\mathbf{p}}_{1}, \overline{\mathbf{p}}_{2}} \circ h_{\overline{\mathbf{p}}_{0}, \overline{\mathbf{p}}_{1}}(x) .
$$

An inverse of a comparison map, a composition of comparison maps, and the union of comparison maps, is a comparison map, if the domain is standard. Thus $\operatorname{map}\left(x, y, \overline{\mathbf{p}}_{0}, \overline{\mathbf{p}}_{3}\right)$ defines a comparison map from $M_{\overline{\mathbf{p}}_{0}}$ to $M_{\overline{\mathbf{p}}_{3}}$; we need to see that this is always total. Well, theorem 2.3 implies the existence of standard low $M_{\overline{\mathbf{p}}_{1}}$ 
and $M_{\overline{\mathbf{p}}_{2}}$ such that $\mathbf{r}_{1} \leqslant \mathbf{r}_{0}$ and $\mathbf{r}_{2} \leqslant \mathbf{r}_{3}$. Lowness of $M_{\overline{\mathbf{p}}_{1}}$ and $M_{\overline{\mathbf{p}}_{2}}$ implies that $\chi_{\text {standard }}\left(\overline{\mathbf{p}}_{i}\right)$ holds for $i=1,2$; this follows from lemma 2.5 .

Now totality of $h_{\overline{\mathbf{p}}_{0}, \overline{\mathbf{p}}_{1}}$ and $h_{\overline{\mathbf{p}}_{3}, \overline{\mathbf{p}}_{2}}$ follows from the fact that $\chi_{\text {standard }}\left(\overline{\mathbf{p}}_{i}\right)$ holds for $i=0,3$; totality of $h_{\overline{\mathbf{p}}_{1}, \overline{\mathbf{p}}_{2}}$ follows from the fact that $M_{\overline{\mathbf{p}}_{1}}$ is low and standard.

The last assertion of the theorem follows immediately from theorem 2.3; again any low, standard model satisfies $\chi_{\text {standard }}$.

Theorem 2.6 enables us to interpret in $\mathcal{R}_{\alpha}$, without parameters, an $\omega$-model $M=\left(\mathbb{N}_{M}, \mathbb{R}_{M}\right)$ of second order arithmetic. The first order part $\mathbb{N}_{M}$ is given by identifying all copies $M_{\overline{\mathbf{p}}}$ of the standard model of arithmetic for $\overline{\mathbf{p}}$ satisfying $\chi_{\text {standard }}$, under the isomorphisms given by the formula map. In detail, let $\overline{\mathbf{p}}, \overline{\mathbf{p}}^{\prime}$ satisfy $\chi_{\text {standard }}$, and let $x \in M_{\overline{\mathbf{p}}}, y \in M_{\overline{\mathbf{p}}^{\prime}}$. Let $(x, \overline{\mathbf{p}}) \sim_{\mathbb{N}}\left(y, \overline{\mathbf{p}}^{\prime}\right)$ if $\operatorname{map}\left(x, y, \overline{\mathbf{p}}, \overline{\mathbf{p}}^{\prime}\right)$ holds. $\mathbb{N}_{M}$ is defined as $\sim_{\mathbb{N}}$-equivalence classes. As map defines isomorphisms, $\sim_{\mathbb{N}}$ is a congruence relation for the operations of arithmetic, and so the arithmetical structure of $\mathbb{N}_{M}$ is also definable in $\mathcal{R}_{\alpha}$.

$\mathbb{R}_{M}$ consists of all sets which are coded as $X_{\overline{\mathbf{p}}}$ for $\overline{\mathbf{p}}$ satisfying $\chi_{\text {standard }}$. Namely, for $\overline{\mathbf{p}}, \overline{\mathbf{p}}^{\prime}$ satisfying $\chi_{\text {standard }}$, let $\overline{\mathbf{p}} \sim_{\mathbb{R}} \overline{\mathbf{p}}^{\prime}$ if for all $n<\omega, n^{M_{\overline{\mathbf{p}}}} \in X_{\overline{\mathbf{p}}}$ iff $n^{M_{\overline{\mathbf{p}}^{\prime}}} \in X_{\overline{\mathbf{p}}^{\prime}}$. This relation is definable in $\mathcal{R}_{\alpha}$. We let $\mathbb{R}_{M}$ be the set of $\sim_{\mathbb{R}}$-equivalence classes. For $n<\omega$ and $Y \in \mathbb{R}_{M}, n \in^{M} Y$ if for some (any) $\overline{\mathbf{p}} \in Y, n^{M_{\overline{\mathbf{p}}}} \in X \overline{\mathbf{p}}$. This too is

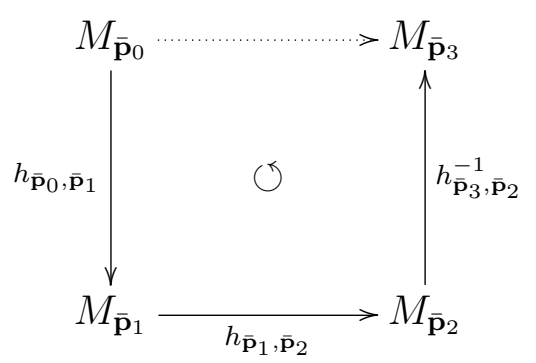

Figure 2.1: The definition of map 
definable in $\mathcal{R}_{\alpha}$. We can quantify over $\mathbb{R}_{M}$ by quantifying over such $\overline{\mathbf{p}}$.

Proof of theorem 1.5. Let $A \subset \omega$ be any $\Sigma_{1}^{1}$ set and suppose that $\alpha>\omega_{1}^{C K}$. In this case, $A$ is $\alpha$-finite, and so there is some $M_{\overline{\mathbf{p}}}$ which codes $A$; so $A \in \mathbb{R}_{M}$. Let $\psi$ be an arithmetical formula such that for all $n<\omega, n \in A$ iff there is some $X \subset \omega$ such that $\psi(n, X)$. We actually know that if $n \in A$ then there is some $X \leqslant_{T} \mathcal{O}$ such that $\psi(n, X)$ holds (this is because the witness $X$ can be taken to be any infinite path in an $\omega$-splitting recursive tree, so we can take the least one). We have $X \in \mathbb{R}_{M}$, and $\psi$ is absolute for $\omega$-models of second order arithmetic, and so $A$ is definable in $M$. It follows that $\operatorname{Th}(\mathbb{N}, A) \leqslant_{1} \operatorname{Th}\left(\mathcal{R}_{\alpha}\right)$. If $A$ is $\Sigma_{1}^{1}$-complete then $\mathcal{O}^{(\omega)} \equiv_{1} \operatorname{Th}(\mathbb{N}, A)$

A very similar proof of theorem 1.5, following [GSS]. By the same argument, $\mathcal{O} \in$ $\mathbb{R}_{M}$. Further, there is some arithmetical formula $\psi$ such that $\mathcal{O}$ is inclusion-wise the least solution of $\psi$ (see $[$ Sac90, p.8]). Again $\psi$ is absolute for $M$ and set inclusion is also definable in $M$ and so this definition of $\mathcal{O}$ holds in $M$.

The case $\alpha=\omega_{1}^{C K}$ has to be treated slightly differently (as is done in [GSS]) because $\mathcal{O}$ is not $\omega_{1}^{C K}$-recursive. Nevertheless even for $\alpha=\omega_{1}^{C K}$ we get $\mathcal{O} \in \mathbb{R}_{M}$ (In fact $\left.\Delta_{2}\left(J_{\alpha}\right) \cap \mathbb{R} \subset \mathbb{R}_{M}\right)$ and the previous arguments hold. We give more details about the required recursion-theoretic constructions (i.e. the proofs of theorems

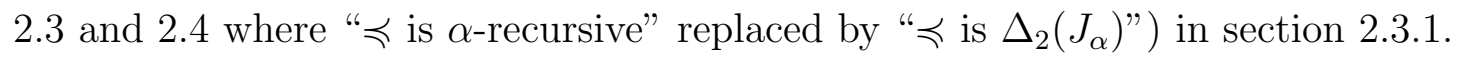

In fact, if $\alpha>\omega_{1}^{C K}$ then iterating this coding shows we can recover more complicated sets. If $\alpha$ is the $n^{\text {th }}$ admissible ordinal then these techniques show that $\mathcal{O}^{\langle n\rangle}$, the $n^{\text {th }}$ iterate of the hyperjump, is in $\mathbb{R}_{M}$. As $\mathcal{O}^{\langle n+1\rangle}$ is definable from $\mathcal{O}^{\langle n\rangle}$ in much the same way that $\mathcal{O}$ was, we can recover it definably in the model 
interpreted in $\mathcal{R}_{\alpha}$. This yields elementary differences between the structures of $\alpha$-r.e. degrees for these ordinals.

This process of coding and recovery can be carried into the transfinite; for example, if $\alpha$ is the $\omega^{\text {th }}$ admissible ordinal then $\mathcal{O}^{\langle\omega\rangle}$ is $\alpha$-finite and so can be coded; it can be recovered by the inductive formula which recovers each of its columns.

\section{$2.2 \quad$ A Template for the Constructions}

The proofs of theorems 2.3 and 2.4 are done by priority constructions which depend on the properties of the underlying admissible ordinal $\alpha$. To avoid repetition and to add rigor, we first present a general template to fit all of these constructions, and prove general facts which are shared by all constructions. Familiarity with the techniques of constructing SW sets in the $\omega$-r.e. degrees, as presented in [NSS98], would be helpful, but we give a complete proof.

From now on we omit the prefix $\alpha$ from the terms 'recursive' and 'r.e.'. Again we remind the reader that the definitions of various notions used in admissible recursion theory (such as strong, weak or nice functionals) are given in appendix A.

\subsubsection{The Requirements}

We are given a recursive partial ordering $\preccurlyeq$ on $\omega$. We construct sets $G_{n}, P, Q$ and $L$. We let $R=\oplus G_{n}$. We strive to meet the following requirements.

1. $T_{n}: Q \leqslant{ }_{\alpha} G_{n} \oplus P$.

2. $M_{\Theta, \Phi, W}$ : If $\Theta(R)=W$ and $\Phi(W \oplus P)=Q$ then there is some $j$ such 
that $G_{j} \leqslant \alpha$. Here $(\Theta, \Phi, W)$ ranges over triples consisting of a strong functional, a weak functional and an r.e. set.

3. $D_{i, j, \Psi}$ : If $j \neq i$, then $\Psi\left(G_{i}\right) \neq G_{j}$. Here $\Psi$ ranges over weak functionals.

4. $N_{i, j, \Psi}$ : If $j \nprec i$ then $\Psi\left(G_{i} \oplus L\right) \neq G_{j}$. $\Psi$ ranges over weak functionals.

5. $K_{\Xi, K}$ : If for all $x \in K$ there are unboundedly many $s$ such that $x \in \Xi(R \oplus$ $P \oplus Q \oplus L)[s]$ then from some $s$ onward, $K \subset \Xi(R \oplus P \oplus Q \oplus L)[s]$ is correct. Here $\Xi$ ranges over enumeration functionals, including ones appearing in the construction with approximations as built in the construction. $K$ ranges over all $\alpha$-finite sets which have a greatest element.

We further ensure that if $j \preccurlyeq i$ then $G_{j} \leqslant \alpha G_{i} \oplus L$.

Constructions proving theorem 2.3 ('type 1 constructions') are also given a nonrecursive r.e. set $A$ (which by Sacks's theorem we may assume is amenable), and try to ensure that $R \leqslant_{\alpha} A$.

Constructions proving theorem 2.4 ('type 2 constructions') are also given a recursive set of $\preccurlyeq$-minimal elements $H$, and uniformly r.e., uniformly amenable arrays $\left\langle U_{i}\right\rangle_{i \in H}$ and $\left\langle V_{i, j}\right\rangle_{i \in H, j<\omega}$, the latter being also uniformly low, such that for all $i \in H$ and $j<\omega, U_{i} \Varangle_{\alpha} V_{i, j}$. We add the requirement

6. $Z_{i, j, \Psi}$ : If $i \in H$, then $G_{i} \neq \Psi\left(V_{i, j}\right)$. $\Psi$ ranges over weak functionals.

We may assume that the enumeration given for $V_{i, j}$ is low for $\Psi$.

We also assume that all functionals given to us are nice. 


\subsubsection{Elements of the Constructions}

All constructions will use a tree of strategies. Each node on the tree (we can always thing of the node as an $\alpha$-finite sequence of ordinals below $\alpha$ ) is an agent, which at various stages of the construction may be assigned requirements for which they work. The lexicographic ordering on the tree is the priority ordering (so an agent $\eta$ is stronger than agent $\rho$ if $\eta \subsetneq \rho$ or if $\eta$ lies on the tree to the left of $\rho$ (lexicographically).) The path of agents accessible at stage $s$ of the construction is always defined, is well-ordered by inclusion and is denoted by $\delta[s]$.

Initialization. The way agents impose finitary-type restraint is by initializing all weaker agents. Whenever an agent enumerates a number into a set or whenever it declares victory, it initializes all weaker agents. Also, at the end of stage $s$ we initialize all agents which lie to the right of $\delta[s]$. Whenever an agent is initialized, all weaker agents are initialized as well.

We also ensure that a change in the requirement to which an agent is assigned entails the initialization of the agent: if $\eta \subset \delta[s]$ and there is no $t<s$ such that $\eta \subset \delta[t]$ and $\eta$ is assigned to a fixed requirement from $t$ to $s$, then we initialize $\eta$ at $s$.

When an agent gets initialized, we cancel all of its followers, chits and pointers (these are various numbers whose proper definitions will shortly follow). All functionals defined by the agent are abandoned. Of course, if a stage $s$ is a limit of stages at which an agent was initialized, then the agent starts afresh at $s$ as well.

We let $\operatorname{init}(\eta)[s]=\sup \{t<s: \eta$ is initialized at $t\}$.

Agreement, Expansion and Restraint. Let $M=M_{\Theta, \Phi, W}$ be a minimality 
requirement. A number $y$ is $M$-confirmed at $s$ if

$$
\Phi(W \oplus P, y) \downarrow=Q(y)[s]
$$

with use $\sigma \oplus \pi=\phi(y)[s]$, and

$$
\sigma \subset \Theta(R)[s]
$$

The length of agreement is

$$
\ell(M)[s]=\max \{z \mid \forall y<z, y \text { is } M \text {-confirmed at } s\}
$$

Suppose that at $s$, agent $\eta \in \delta[s]$ is working for $M$. A stage $s$ is $\eta$-expansionary if for all $t<s$ such that $\eta \in \delta[t], \ell(M)[t]<\ell(M)[s]$.

At stage $s$, if agent $\eta$ is working for $M$, we let

$$
r(\eta)[s]= \begin{cases}0 & \text { if } s \text { is } \eta \text {-expansionary or a limit of } \eta \text {-expansionary stages, } \\ t & \text { if not, and } t=\sup \{u<s: u \text { is } \eta \text {-expansionary }\}\end{cases}
$$

We let $\operatorname{Rest}(\eta)[s]=\sup _{\rho \subsetneq \eta} r(\rho)[s]$ (agents $\rho$ which lie to the left of $\eta$ impose restraint by was of initialization so $\eta$ does not have to take $r(\rho)$ into consideration for such $\rho)$.

$T$ Requirements. Suppose that agent $\eta$ is assigned to $T_{n}$. The agent $\eta$ defines a functional $\Gamma_{\eta}$, with the intention of having $\Gamma_{\eta}\left(G_{n} \oplus P\right)=Q \cdot \eta$ may only add an axiom to $\Gamma_{\eta}$ when it is accessible.

All uses of $\Gamma$ functionals are successor ordinals; $\gamma_{\eta}(x)=\gamma_{\eta}\left(G_{n} \oplus P, x\right)$ denotes the length of the use of the computation $\Gamma_{\eta}\left(G_{n} \oplus P, x\right)$ minus one, so enumerating it into $G_{n}$ or $P$ destroys the computation.

The following is important.

Definition. When we design the tree and assign requirements to agents, we always make sure that for every agent $\eta$ (and at every stage $s$ ), only finitely many agents 
$\rho \subset \eta$ are assigned to some $T_{n}$ requirement at $s$. We let $n(\rho)[s]<\omega$ be the maximum of $n$ such that some $\eta \subset \rho$ is assigned to $T_{n}$ (at $s$ ).

Followers. Agents working for $D, N$ or $Z$ requirements may have followers. A follower $x<\alpha$ for an agent $\eta$ working for $D_{i, j, \Psi}$ is targeted for $G_{j}$ and for $L$. It is realized at $s$ if $\Psi\left(G_{i}, x\right) \downarrow=0[s]$. If $\eta$ works for $N_{i, j, \Psi}$ then $x$ is targeted for $G_{k}$ for every $k$ such that $j \preccurlyeq k$ (including of course $j$ itself). In this case $x$ is realized if $\Psi\left(G_{i} \oplus L, x\right) \downarrow=0[s]$.

In type 2 constructions, a follower $x$ for an agent $\eta$ working for $Z_{i, j, \Psi}$ is targeted for $G_{i}$ and for $L$; it is realized if $\Psi\left(V_{i, j}, x\right) \downarrow=0[s]$.

In type 1 constructions, a follower $x$ (for any agent) is permitted at $s$ if some number $y<x$ enters $A$ at $s$.

In type 2 constructions, if $x$ is a follower targeted for $G_{i}$ and $i \in H$ then $x$ is permitted at $s$ if some number $y<x$ enters $U_{i}$ at $s$. In these constructions, if $x$ is not targeted for $G_{i}$ for any $i \in H$ then $x$ is always permitted. Note that by our instructions, and by the fact that $H$ is a set of $\preccurlyeq$-minimal elements, a follower $x$ may be targeted for $G_{i}$ for at most one $i \in H$.

Pointers. An agent $\eta$ working for a requirement $K_{\Xi, K}$ keeps a pointer $i(\eta)[s]$, which is the next element of $K$ of which it needs to take care. Unless initialized, at a limit stage $s$ we have $i(\eta)[s]=\min \left(K \backslash \sup \{i(\eta)[t]\}_{t<s}\right)$. When $\eta$ is initialized we set $i(\eta)=\min K$. If $\eta$ acts at $s$ then we update the pointer: $i(\eta)[s+1]=$ $\min (K \backslash i(\eta)[s]+1)$. If $i(\eta)[s]=\max K$ and $\eta$ acts at $s$ then $\eta$ declares victory.

Chits. Suppose that agent $\eta$ works for $M=M_{\Theta, \Phi, W} \cdot \eta$ defines weak functionals $\Delta_{\eta, j}$ for every $j \leqslant n(\eta)$, with intended oracle $W$; the intention is that if the hypothesis of $M$ holds then for some $j \leqslant n(\eta)$ we'll have $\Delta_{\eta, j}(W)={ }^{*} G_{j}$. 
$\eta$ is allowed to extend the definition of $\Delta_{\eta, j}(W)$ (i.e. to enumerate a new axiom into $\left.\Delta_{\eta, j}\right)$ at stages which are $\eta$-expansionary. As is implies by the intention, $\eta$ always defines the value of $\Delta_{\eta, j}(W, x)[s]$ to be $G_{j}(x)[s]$.

$\eta$ makes $\Delta_{\eta, j}$ monotone, so that at any stage we'll have dom $\Delta_{\eta, j}(W)$ an ordinal. If $\eta$ wishes to extend $\Delta_{\eta, j}$ at $s$ then it defines one new axiom on the input $x=$ $\operatorname{dom} \Delta_{\eta, j}(W)[s]$ (we'll ensure that the use is larger than the uses $\delta_{\eta, j}(W, y)[s]$ for all $y<x$ so that indeed monotonicity is maintained.)

To each computation $(\sigma ; x, l) \in \Delta_{\eta, j}$ is associated a chit $(y, \pi)$ (we sometimes also refer to $y$ as the chit). When $\eta$ wishes to define $\Delta_{\eta, 0}(x)$ at stage $s$, it picks a new suitable chit. A chit $(y, \pi)$ is suitable to be picked for a new computation $\Delta_{\eta, 0}(W, x)[s]$ if:

1. $y \in \alpha^{[\eta]}$.

2. $y<\ell(M)[s]$.

3. $\phi(W \oplus P, y)[s]=\sigma \oplus \pi$.

4. $y>\operatorname{init}(\eta)[s]$ and $y>t$ for any $t<s$ at which $\eta$ defined any $\Delta_{\eta, 0}$ computation.

If $s$ is $\eta$-expansionary and there is a suitable chit $y$, then it defines $\Delta_{\eta, 0}(W, x)$ with use $\sigma$.

Now let $j>0$. Suppose that at $s, \eta$ wishes to define $\Delta_{\eta, j}(W, x)$. To do so, it needs to find a chit $(y, \pi)$ which is suitable to be picked for this computation (we also say that the chit is $j$-suitable). The suitability conditions are:

1. $(y, \pi)$ is a chit for a computation $\Delta_{\eta, j-1}\left(W, x^{\prime}\right)[s]$ (whose use is $\left.\sigma\right)$. 
2. Further, that chit is still active, which means that $\pi \subset P[s]$ (an inactive chit is also called cancelled).

3. The computation $\Delta_{\eta, j-1}\left(W, x^{\prime}\right)[s]$ is failed, which means that its value disagrees with $G_{j-1}\left(x^{\prime}\right)$ (necessarily $x^{\prime}$ entered $G_{j-1}$ at some stage after the computation was defined).

4. The size condition: $y>t$ for any $t<s$ at which $\eta$ defined any $\Delta_{\eta, j}$ computation.

If such a chit is found then $\eta$ defines $\Delta_{\eta, j}(W, x)[s]$ with the same use $\sigma$ as the accompanying computation $\Delta_{\eta, j-1}\left(W, x^{\prime}\right)[s]$.

If a computation $\Delta_{\eta, j}(W, x)$ which is defined at $s$ becomes incorrect at a later stage $t$ (that is some number smaller than the use enters $W$ ) then the accompanying chit $(y, \pi)$ is cancelled and never considered again. Note again that if $(y, \pi)$ is a chit for $\Delta_{\eta, j}\left(W, x_{j}\right)$ for $j \leqslant i$ (where $i \leqslant n(\eta)$ ) then the uses of all of these computations are the same and so all such computations disappear together). However, (2) shows that the chit may be cancelled even if the computations still hold.

At stage $s, \eta$ may wish to use a chit $(y, \pi)$ for purposes of victory. Suppose that $\rho \subset \eta$ is an agent which works for $T_{n}$ at $s$. A chit $(y, \pi)$ is cleared by $\rho$ at stage $s$ if $\neg\left(\Gamma_{\rho}\left(B \oplus G_{n} \oplus P, y\right) \downarrow=0[s]\right)$, or if $\Gamma_{\rho}\left(B \oplus G_{n} \oplus P, y\right) \downarrow=0[s]$ with use $\gamma_{\rho}(y)[s]>\operatorname{dom} \pi$. The chit $y$ is victorious if it is $M$-confirmed at $s$, still active, is greater than $\operatorname{Rest}(\eta)[s]$, and is cleared by all such agents $\rho$ below $\eta$.

\section{Guessing}

This pertains only to type 2 constructions. The fact that $V_{i, j}$ are uniformly low implies that there are recursive functions $f, g$ with the following properties: for all 
$i, j, \Psi, \eta, \lim _{s \rightarrow \alpha} f\left(Z_{i, j, \Psi}, s\right)$ and $\lim _{s \rightarrow \alpha} g\left(\eta, Z_{i, j, \Psi}, s\right)$ exist and are either yes or no. Also,

$\lim _{s \rightarrow \alpha} f\left(Z_{i, j, \Psi}, s\right)=$ yes iff there is some stage $s$ of the construction and some $x \in G_{i}[s]$ which at $s$ is realized $\left(\Psi\left(V_{i, j}, x\right) \downarrow=0\right)$ by a correct computation;

$\lim _{s \rightarrow \alpha} g\left(\eta, Z_{i, j, \Psi}, s\right)=$ yes iff there is some stage $s$ of the construction at which $\eta$ works for $Z_{i, j, \Psi}, \eta$ is eligible to act and does not believe it is satisfied, and $\eta$ has a follower $x$ which is permitted and is realized by a correct computation.

The recursion theorem allows us to use these function during the construction.

Suppose that at stage $s, \eta$ is an agent working for $Z_{i, j, \Psi}$. Suppose that $x \in G_{i}[s]$ is realized at $s$. To check whether $\eta$ believes this realization is correct, it looks for the least stage $t>s$ at which either the computation realizing $x$ at $s$ is discovered to be incorrect, or $f(Z, t)=$ yes. If it gets the latter outcome then $\eta$ believes the realization and believes that it is satisfied and does not need to act.

Suppose that $\eta$ is eligible to act at $s$ and that at $s, \eta$ has a follower $x$ which is realized and permitted. Before acting, $\eta$ makes a guess about $x$ 's realization in much the same way: it looks for $t>s$ at which this realization is discovered to be incorrect, or $g(\eta, Z, t)=$ yes. Again $\eta$ believes this realization if the latter is the outcome.

\subsubsection{Construction}

The structure of every stage in all constructions is fixed. Every stage consists of two pahses: at the first, agents who have finitary-type actions to perform may do so (these actions need to be performed immediately, and the agents cannot wait 
until they are accessible.) At the second phase, less pressing matters are dealt with.

\section{The First Phase}

At the first phase, agents working for finitary-type requirements - D, $N, Z, K$ requirements - that wish to act and are eligible to act, are allowed to act. An agent $\eta$ is eligible to act at the first phase of stage $s$ if there was a stage $t<s$ such that $\eta$ was accessible at $t$, and such that between $t$ and $s$, neither was $\eta$ initialized, nor did it act.

No agent $\eta$ wishes to act at $s$ (at either phase) if it declared victory since $\operatorname{init}(\eta)[s]$.

$K$. Suppose that at stage $s, \eta$ works for $K_{\Xi, K} . \eta$ wishes to act at $s$ if $i(\eta)[s] \in$ $\Xi(R \oplus P \oplus Q \oplus L)[s]$. To act, it initializes weaker agents. As described above, after acting $\eta$ either updates $i(\eta)$ or declares victory.

$N$ and $D$. Suppose that at $s, \eta$ works for some $N$ or $D$ requirement. $\eta$ may have three reasons to wish to act:

1. It has a follower which is both realized and permitted.

2. It has a follower which is realized for the first time.

3. All of its followers are realized (this includes the possibility that there are no followers).

$\eta$ 's action corresponds to the case of affairs:

1. $\eta$ enumerates the follower into the sets it is targeted for and declares victory.

2. $\eta$ initializes weaker agents. 
3. $\eta$ appoints a new, large follower (taken from $\alpha^{[\eta]}$ ), and initializes weaker agents.

$Z$. Suppose that at $s, \eta$ works for some $Z$ requirement. $\eta$ first guesses if it is already satisfied. If not, and if $\eta$ has some follower that is both permitted and realized, then $\eta$ guesses whether this realization is correct; if so, it wishes to act and indeed enumerates the follower into the sets for which it is targeted. It also initializes weaker agents.

If it didn't act, then $\eta$ may yet wish to act if all of its followers are realized at $s$ (it does not perform further guesses). In this case it appoints a new, large follower and initializes weaker agents.

We need to note something important. It may seem futile to let every agent that wishes to act at the first phase the right to do so, as action by a stronger requirement will immediately initialize it. This is correct, except for agents working for $Z$ requirements. They may succeed even if initialized, and for the tactic of low guessing to succeed we need to allow them to act whenever they wish. Furthermore, the priority ordering on all agents may not be a well-ordering, so there isn't necessarily a strongest agent wishing to act. [This leads to intriguing consequences. Suppose we wished to overcome the first difficulty by adding the phrase "and no stronger requirement wishes to act" to the properties of $g$ (and thus of the guessing). Suppose further that there is an infinite descending chain of agents for $Z$ requirements which have realized, permitted followers ready for the testing. The agents now have no idea whether they should perform the guess; if the guess for a stronger agent succeeds then their own guess may never terminate.] 


\section{The Second Phase}

At the second phase, all agents which are currently accessible and which work for an $M$ requirement or a $T$ requirement follow the following orders. We note that $\delta[s]$ is well-ordered, so if some agent wishes to declare victory and initialize weaker agents then we let the strongest one do so.

$M$. Suppose that at stage $s, \eta$ works for $M=M_{\Theta, \Phi, W}$. As usual, if $\eta$ declared victory since $\operatorname{init}(\eta)[s]$ then it does absolutely nothing.

If there is some $\sigma \subset \Theta(R)[s]$ and some $x<\operatorname{dom} \sigma$ such that $\sigma(x)=0$ and $x \in W[s]$ then $\eta$ declares victory (this is called "easy victory").

If easy victory was not declared, and if $r(\eta)[s]>0$, then $\eta$ does nothing at this stage. Otherwise, $\eta$ looks for victorious chits. If there is one, it enumerates the least one $y$ into $Q$, and for all $\rho \subset \eta$, if $\Gamma_{\rho}\left(G_{n(\rho)} \oplus P, y\right) \downarrow=0[s]$, it puts $\gamma_{\rho}\left(G_{n(\rho)} \oplus P, y\right)[s]$ into $P ; \eta$ declares victory.

If $\eta$ hasn't won yet, and the stage is $\eta$-expansionary, then $\eta$ tries to extend $\Delta_{\eta, j}(W)$ as described above. Suppose $\eta$ just defined $\Delta_{\eta, j}(x)$. Suppose that $\rho \supset \eta$ is an agent extending $\eta$ which works for some $D, Z$ or $N$ requirement and has a follower $x$, targeted for $G_{j}$. Then $\rho$ now initializes all weaker agents.

$T$. If $\eta \in \delta[s]$ works for $T_{n}$ at $s$, then $\eta$ extends $\Gamma_{\eta}$ : it finds the least $x$ such that $\Gamma_{\eta}\left(G_{n} \oplus P, x\right) \uparrow[s]$, and sets $\Gamma_{\eta}\left(G_{n} \oplus P, x\right) \downarrow=Q(x)[s]$ with large use.

\section{What we didn't specify}

We note that in order to specify a construction, all we need to do is specify what the tree of agents is, how to calculate $\delta[s]$ and how to assign requirements to agents at each stage; and we need to verify that $n(\rho)<\omega$ for every agent $\rho$. 


\subsubsection{Verifications}

We first remark that the properties of the functions $f$ and $g$ ensure that every particular search must terminate by a bounded amount of time; either the realization of the number $x$ being tested is incorrect, or $x$ and $s$ are witnesses to the limit value being yes.

It follows that the searching period at the beginning of the first phase of every stage does indeed take only $\alpha$-finitely much time. For the function taking each instance of guessing to the stage at which the guess is resolved is recursive, and at each stage only $\alpha$-finitely many guesses are made.

\section{Fairness Attempted}

Suppose that $\eta$ is an agent that eventually stops being initialized:

$$
r^{*}=\operatorname{init}(\eta)[\alpha]<\alpha .
$$

It follows that $\eta$ works for a fixed requirement after $r^{*}$ (or works for no requirement at all). Further assume that $\eta$ is accessible unboundedly often.

We examine how $\eta$ affects weaker requirements.

$M$. If $\eta$ works for an $M$ requirement then $\eta$ initializes weaker agents at most once after $r^{*}$ (when declaring victory).

$K$. Suppose that $\eta$ works for the requirement $K_{\Xi, K}$. After $r^{*}, i(\eta)[s]$ is nondecreasing. Let $K^{\prime}=\left\{i(\eta)[s]: s>r^{*}\right\} ; K^{\prime}$ is $\alpha$-finite as it is an initial segment of $K$. The function which takes $x \in K^{\prime}$ to the least $s>r^{*}$ at which $i(\eta)[s]=x$ is recursive and so bounded by some $s^{*}$. After $s^{*}, \eta$ does not initialize weaker agents.

Suppose that $\eta$ works for a $D$ or an $N$ requirement. 
Observation 2.7. If $x$ is a follower for $\eta$ which is realized at $s>r^{*}$, then this realization is preserved by $\eta$ 's initializing of weaker agents at $s$.

Lemma 2.8. Eventually, $\eta$ stops appointing new followers.

Proof. This is a standard permitting argument. If $\eta$ declares victory at $s>r^{*}$ then after $s, \eta$ ceases all action. Otherwise: suppose we are dealing with a type 1 construction. If at stage $s>r^{*}, x$ is a follower for $\eta$ which is realized, then $A\lceil x[s]=A\lceil x$. This is because $x$ remains realized and $\eta$ does not act; so $x$ is never permitted after $s$. If the lemma fails then $\eta$ keeps appointing followers which are unbounded in size (as it appoints large followers), and each follower eventually gets realized (by the time a larger follower gets appointed). This allows us to compute $A$.

If this is a type 2 construction and $\eta$ appoints followers targeted for $G_{i}, i \in H$, then this argument holds, with $U_{i}$ in place of $A$. If $\eta$ does not target followers for $G_{i}$ for any $i \in H$, then $\eta$ only appoints one follower after $r^{*}$ : whenever a follower gets realized it can be enumerated.

Suppose that $\eta$ works for $Z=Z_{i, j, \Psi}$.

Lemma 2.9. $\eta$ eventually stops appointing new followers or enumerating followers into $G_{i}$.

Proof. This is classical. If $\lim f(Z, s)=$ yes, then from the stage at which $f(Z, s)$ stabilizes, $\eta$ always guesses itself to be successful and does nothing. If $\lim g(\eta, Z, s)=$ yes then $\lim f(Z, s)=$ yes; the witness for the former is believed useful and enumerated.

Suppose that $\lim g(\eta, Z, s)=\lim f(Z, s)=$ no and both stabilize after $t>r^{*}$. Then after $t$, no follower is deemed useful, so $\eta$ doesn't enumerate followers after 
$t$.

Suppose that $\eta$ keeps appointing new followers. Let $x$ be a follower for $\eta$, appointed at $s>r^{*}$. At every stage $u>s$ at which $\eta$ appoints another follower, $x$ is realized. The enumeration $\left\langle V_{i, j}[s]\right\rangle$ is low for $\Psi$ so $x$ is eventually correctly realized. After $t^{*}$, a correctly realized follower cannot be permitted: $\eta$ does not believe itself successful after $t^{*}$ and so a correctly realized, permitted follower would be a witness for $\lim _{s \rightarrow \alpha} g(\eta, Z, s)=$ yes. As the size of followers appointed is unbounded, this gives us a procedure of computing $U_{i}$ from $V_{i, j}$.

It follows that if $\eta$ works for a $D, N$ or $Z$ requirement, then $\eta$ eventually stops initializing weaker requirements on its own accord. We still have to verify it stops initializing weaker requirements when it is instructed to do so by a stronger agent. Suppose then that $\rho \subsetneq \eta$ works for $M=M_{\Theta, \Phi, W}$, fix $j \leqslant n(\rho)$ and suppose that $\eta$ targets followers for $G_{j}$.

Observation 2.10. Suppose that $s>t>\operatorname{init}(\rho)[\alpha]$. Suppose that $\Delta_{\rho, j}(W, x) \downarrow[t]$ with use $\sigma$, and that $\sigma \subset \Theta(R)[s]$. Then $\rho$ does not redefine $\Delta_{\rho, j}(x)$ at stage $s$. For if it does, then $\Delta_{\rho, j}(W, x) \uparrow[s]$ which means that $\sigma \not \subset W[s]$ anymore; necessarily some $y<\operatorname{dom} \sigma$ entered $W$ between $t$ and $s$. But then $\rho$ can get an easy victory at $s$ and defines nothing.

Let $K(\eta)$ be the final set of followers for $\eta$ (it is $\alpha$-finite), and let $r^{* *}>r^{*}$ be a stage after which $\eta$ neither appoints any followers, nor enumerates followers into sets. Suppose that $x \in K(\eta)$ and suppose that at some stage $s>r^{* *}, \rho$ defines $\Delta_{\rho, j}(x)$, say with use $\sigma$. At some stage $t>r^{*}, x$ is chosen as a follower for $\eta$. Since large followers are chosen, we must have $t<s$; so $\eta$ initializes weaker agents at $s$. $\eta$ 's action at $s$ ensures that $\sigma \subset \Theta(R)$ from $s$ onwards (this is because $s>r^{* *}$ ). It follows that $\rho$ will not redefine $\Delta_{\rho, j}(x)$ at any other stage after $r^{* *}$. 
Let $K^{\prime}(\eta, \rho, j)$ be the set of followers $x \in K(\eta)$ such that $\eta$ initializes for the sake of a $\Delta_{\rho, j}(x)$ computation after stage $r^{* *}$. Let $x_{0}=\min K^{\prime}(\eta, \rho, j), x_{2} \in$ $K^{\prime}(\eta, \rho, j)$ and $x_{1} \in K(\eta) \cap\left(x_{0}, x_{1}\right)$. Say that $\eta$ initializes on behalf of $\Delta_{\rho, j}\left(x_{0}\right)$ at $s_{0}>r^{* *}$ and on behalf of $\Delta_{\rho, j}\left(x_{2}\right)$ at $s_{2}>r^{* *}$. By the above analysis, and since $\Delta_{\rho, j}\left(W, x_{0}\right) \downarrow\left[s_{2}\right]$, we know that $s_{2}>s_{0}$ and that at some stage $s_{1} \in\left(s_{0}, s_{2}\right), \rho$ defines $\Delta_{\rho, j}\left(x_{1}\right)$. It follows that $x_{1} \in K^{\prime}(\eta, \rho, j)$. Thus $K^{\prime}(\eta, \rho, j)$ is the intersection of $K(\eta)$ with an interval, and so is $\alpha$-finite.

For $x \in K^{\prime}(\eta, \rho, j)$, let $s_{\eta, \rho, j}(x)$ be the (unique) stage at which $\rho$ defines $\Delta_{\rho, j}(x)$ (and $\eta$ initializes for $x$ ). As $K^{\prime}(\eta, \rho, j)$ is $\alpha$-finite, $s_{\eta, \rho, j}$ " $K^{\prime}(\eta, \rho, j)$ is bounded. We get:

Lemma 2.11. Let $\eta$ be any agent such that init $(\eta)[\alpha]<\alpha$. There is a stage after which $\eta$ does not initialize weaker requirements on its own behalf. If $\eta$ works (after $\operatorname{init}(\eta)[\alpha]$ ) for a $D, N$ or a $Z$ requirement, then for every $\rho \subset \eta$ there is a stage after which $\eta$ does not initialize on $\rho$ 's behalf.

\section{The Fairness Assumption}

We now make the fairness assumption on the construction:

For every requirement $R$ there is an agent $\eta$ which is not initialized unboundedly often $(\operatorname{init}(\eta)[\alpha]<\alpha$ ), is accessible unboundedly often, and which eventually works for $R$. Furthermore, if $R$ is an $M$ requirement, then there are club many stages at which $\eta$ is accessible and on which $\operatorname{Rest}(\eta)$ is bounded.

We show that if the assumption holds then the construction succeeds. 


\section{Success of the Finitary-Type Requirements}

Lemma 2.12. Every $K$ requirement is met.

Proof. Suppose that $\eta$ is the agent working for $K_{\Xi, K}$. If $\eta$ acts at $s>\operatorname{init}(\eta)[\alpha]$ then $i(\eta)[s] \in \Xi(R \oplus P \oplus Q \oplus L)$ is permanently correct. Thus if $\eta$ declares victory after $\operatorname{init}(\eta)[\alpha]$ then it succeeds.

If not, let $i$ be the final value of $i(\eta)[s]$, stabilizing after $s^{*}$. For no $s>s^{*}$ do we have $i \in \Xi(R \oplus P \oplus Q \oplus L)[s]$ and so the requirement is met vacuously.

Corollary 2.13. All sets constructed are amenable.

Proof. Fix $\beta<\alpha$. Let $\Xi=\{p: \operatorname{dom} p>\beta\}$. Success of $K_{\Xi}$ implies that there is some stage $s<\alpha$ after which $R \oplus P \oplus Q \oplus L \uparrow \beta$ is fixed.

Corollary 2.14. All sets constructed are low, hence admissible.

Proof. We show that some functional is low for $X=R \oplus P \oplus Q \oplus L$. Fix some $\Xi$ such that $\Xi(X)=X^{\prime}$. Success of $K_{\Xi,\{x\}}$ for all $x<\alpha$ shows that $\Xi$ is weakly low for $X$. Suppose further that $K$ is $\alpha$-finite; we want to show that if $K \subset X^{\prime}$ then from some stage we have $K \subset \Xi(X)[s]$. If $K$ has a maximal element then this is ensured by the success of $K_{\Xi, K}$. Otherwise, this is ensured by the success of $K_{\Xi^{\prime}, K^{\prime}}$ where $K^{\prime}=K \cup\{\sup K\}$ and $\Xi^{\prime}=\Xi \cup\{(\langle\rangle, \sup K)\}$.

Let $\eta$ be the agent working for $T_{n}$.

Claim 2.15. If $\Gamma_{\eta}\left(G_{n} \oplus P, y\right) \downarrow$ then $\Gamma_{\eta}\left(G_{n} \oplus P, y\right)=Q(y)$.

Proof. Let $s>r^{*}=\operatorname{init}(\eta)[\alpha]$, and suppose that $\Gamma_{\eta}\left(G_{n} \oplus P, y\right) \downarrow[s]$ and that at $s$, agent $\rho$, working for $M_{\Theta, \Phi, W}$ puts $y$ into $Q . \rho$ cannot be stronger than $\eta$ since 
$s>r^{*}$. Also, $\rho$ cannot be to the right of $\eta ; \eta$ defined $\Gamma_{\eta}(y)$ at stage $t<s$ and initialized all nodes to its right at $t$; and new chits $y$ are picked large. Thus $\eta \subset \rho$.

Therefore $\rho$ puts $\gamma_{n}(y)[s]$ into $P$ at $s$, removing the computation $\Gamma_{\rho}\left(G_{n} \oplus P, y\right)$. If the computation is defined later then the value must be correct.

Claim 2.16. Suppose that $\beta<\alpha$ and that $\Gamma_{\eta}\left(G_{n} \oplus P\right)\lceil\beta$ stabilizes by some $\alpha$-finite stage. Then $\Gamma_{\eta}\left(G_{n} \oplus P, \beta\right) \downarrow$.

Proof. Suppose that after $t>r^{*}, \Gamma_{\eta}\left(G_{n} \oplus P\right) \uparrow \beta$ is fixed. At every later stage at which $\eta$ is allowed to define $\Gamma_{\rho}$, if $\Gamma_{\rho}\left(G_{n} \oplus P, \beta\right) \uparrow$ then it gets defined, say with use $\tau$. At such a stage enumerate $\tau$ into a functional $\Xi$. Let $\rho$ be the agent which works for $K_{\Xi}$. At some stage $t>s, \Xi$ acts and so preserves $\tau \subset G_{n} \oplus P$.

Lemma 2.17. Every $T$ requirement is met.

Proof. For every $\beta, \Gamma_{\eta}\left(G_{n} \oplus P\right) \uparrow \beta$ eventually stabilizes. This follows from the fact that $G_{n} \oplus P$ is admissible and is proved by induction. Assume up to $\beta$; the function taking $y<x$ to the stage at which the correct computation $\Gamma_{\eta}\left(G_{n} \oplus P, y\right)$ is defined is weakly recursive in $G_{n} \oplus P$ and so is bounded.

It follows that $\Gamma_{\eta}\left(G_{n} \oplus P\right)=Q$. As $G_{n} \oplus P$ is admissible, $Q \leqslant{ }_{\alpha} G_{n} \oplus P$.

Let $\eta$ be the agent responsible for a $D$ or an $N$ requirement, and let $K(\eta)[s]$ be the set of followers $\eta$ has at stage $s>r^{*}(K(\eta)=K(\eta)[\alpha])$.

Claim 2.18. Every $x \in K(\eta)[s]$ is realized at $s$, except perhaps for a maximal element.

Proof. We show this by induction on $s$. Assume that $s$ is a limit stage and that $x \in K(\eta)[s]$ is not maximal. Take some $y>x, y \in K(\eta)[s]$. For some $t<s$, 
$x, y \in K(\eta)[t]$. By induction, $x$ is realized at $t$. By observation 2.7, $x$ is realized at $s$ as well.

Assume the statement holds for $K(\eta)[s]$. Then it holds for $K(n)[s+1]$, because at stage $s$ all previously realized numbers are still realized (same observation), and $\eta$ perhaps appoints a new follower, larger than $\sup K(\eta)[s]$, but only if every $x \in K(\eta)[s]$ is realized.

Lemma 2.19. Every $D$ and $N$ requirement succeeds.

Proof. If $\eta$ declares victory after $r^{*}=\operatorname{init}(\eta)[\alpha]$ then the diagonalization is preserved. Otherwise, suppose that after $t>r^{*}, \eta$ appoints no new followers. This means that there is one maximal follower $x$ which is never realized. Success follows.

Lemma 2.20. Every $Z$ requirement succeeds.

Proof. Let $\eta$ be the agent working for $Z=Z_{i, j, \Psi}$. If $\lim f(Z, s)=1$ then success is clear. If not, let $K(\eta)$ be the set of final followers (that are not enumerated into $\left.G_{i}\right)$; after some $t>\operatorname{init}(\eta)[\alpha], \eta$ does not enumerate any followers or appoint new ones.

We claim that there is some $x \in K(\eta)$ which is not realized (at $\alpha$ ). Otherwise, there is a stage at which all permanent followers are correctly realized: the function taking the follower to the stage at which it is correctly realized is computable from $V_{i, j}$, which is admissible, and $K(\eta)$ is $\alpha$-finite. But after that stage, $\eta$ would appoint a new follower: it does not believe itself to be successful.

Suppose that $j \preccurlyeq i$. We want to show that $G_{j} \leqslant_{w \alpha} G_{i} \oplus L$. To determine whether $x \in G_{j}$, we first wait until stage $x$ to see if $x$ was chosen as a follower for 
some agent $\eta$ which targets $x$ to $G_{j}$. If not then $x \notin G_{j}$. Suppose that it is. If $\eta$ works for $D_{k, j, \Psi}$ or $Z_{j, k, \Psi}$ then $x \in G_{j}$ iff $x \in L$. If $\eta$ works for $N_{k, l, \Psi}$ (so $\left.l \preccurlyeq j\right)$ then $x \in G_{j}$ iff $x \in G_{i}$ (since $l \preccurlyeq i$ too). Admissibility implies that $G_{j} \leqslant_{\alpha} G_{i} \oplus L$. Overall we see that indeed the partial ordering coded by $L$ is $\preccurlyeq$.

Finally we mention that the permitting method indeed ensures that in type 1 constructions, $R, L \leqslant_{\alpha} A$, and that in type 2 constructions, $G_{i} \leqslant_{\alpha} U_{i}$ for $i \in H$.

\section{The Minimality Requirements}

Let $\eta$ be the agent working for $M=M_{\Theta, \Phi, W}$, and let $r^{*}=\operatorname{init}(\eta)[\alpha]$.

Observation 2.21. Suppose that $s>t>r^{*}$. Suppose that at $t,(y, \pi)$ is picked as a chit for a computation $\Delta_{\eta, 0}(x)$ with use $\sigma$. Suppose that at $s,(y, \pi)$ is transferred as a chit for a $\Delta_{\eta, j}$ computation. Definitions of $\Delta$ computations are done only at $\eta$-expansionary stages, so $y<l(M)[t]<l(M)[s]$, so $y$ is $M$-confirmed at $s$. Also, $(y, \pi)$ is still active at $s$. This implies that $\phi(W \oplus P, y)[s]=\sigma \oplus \pi$ (we use niceness of $\Phi$ here). Thus $\sigma \subset \Theta(R)[s]$. Note that once the chit is cancelled it is never active again (and will never be picked again as a new chit for $\Delta_{\eta, 0}$ as we pick large chits).

Lemma 2.22. If $\eta$ declares victory after $r^{*}$, then the hypotheses of $M$ do not hold.

Proof. If easy victory is declared then $\eta$ preserves a discrepancy between $\Theta(R)$ and $W$. Suppose that at $s>r^{*}, \eta$ enumerates a victorious chit $y$ into $Q$. Let $\sigma \oplus \pi=\phi(W \oplus P, y)[s] . \sigma \subset \Theta(R)[s]$ holds by observation 2.21 and is preserved by $\eta$ 's action at $s$ (so if $\sigma \not \subset W$ we again get an easy win.) We claim that $\pi \subset P$ is preserved (and so that $y$ is not $M$-confirmed at $\alpha$ ). The only obstacle can be the uses of the form $\gamma_{\rho}\left(G_{n} \oplus P, y\right)$ which $\eta$ enumerates into $P$ as it declares victory. 
A use of this kind is enumerated only if $\Gamma_{\rho}$ clears $y$ at $s$, thus all $\gamma_{\rho}\left(G_{n} \oplus P, y\right)$ enumerated are greater than dom $\pi$.

The following lemma will be needed here and also in the following section in order to ensure that the fairness assumption holds.

Claim 2.23. $r(\eta)[s]$ is bounded on a recursive club.

Proof. $r(\eta)[s]$ is constant on a recursive club: if there are unboundedly many $\eta$ expansionary stages, then the set of stages at which $r(\eta)=0$ is a (recursive) club. If not, then $r(\eta)[s]$ is eventually constant.

Assume that the hypotheses of $M$ hold.

Claim 2.24. $\lim _{s \rightarrow \alpha} l(M)[s]=\alpha$.

Proof. This is because $W \oplus P \oplus R$ is admissible. The least stage at which a number $x$ is $M$-confirmed with correct uses for $\Theta(R)$ and $\Phi(W \oplus P)$ is computable (as a function of $x$ ) from $W \oplus P \oplus R$; hence bounded on initial segments of $\alpha$.

It follows that there are unboundedly many $M$-expansionary stages.

Let $n=n(\eta)$.

Claim 2.25. Let $j \leqslant n$. For all $x$, there is a stage after which $\eta$ stops defining $\Delta_{\eta, j}(x)$

Proof. At each stage $s$ at which $\eta$ defines $\Delta_{\eta, j}(x)$, say with use $\sigma$, enumerate $\theta(R ; \operatorname{dom} \sigma)[s]$ into a functional $\Xi$ (recall observation 2.21). The success of the agent $\rho$ which works for $K_{\Xi}$ ensures that if unboundedly many attempts at defining $\Delta_{\eta, j}(x)$ are made, then $\theta(R ; \operatorname{dom} \sigma)[s]$ for some such definition will be preserved. By observation 2.10, $\Delta_{\eta, j}(x)$ doesn't get redefined after $s$. 
Claim 2.26. $\operatorname{dom} \Delta_{\eta, 0}(W)=\alpha$.

Proof. By induction on $x$, we show that $\Delta_{\eta, 0}(W, x) \downarrow$. If $x \leqslant \operatorname{dom} \Delta_{\eta, 0}(W)$, then by admissibility of $W$, we know that $\Delta_{\eta, 0}(W) \uparrow x$ eventually stabilizes. It is enough now to show that for unboundedly many $s, \Delta_{\eta, 0}(W, x) \downarrow[s]$; by claim 2.25 , after some $t, \eta$ stops defining $\Delta_{\eta, 0}(x)$, hence all computations $\Delta_{\eta, 0}(W, x)[s]$ for $s>t$ must be the same computation, which is permanent.

Suppose that by $s^{*}>r^{*}, \Delta_{\eta, 0}(W)\left\lceil x\right.$ is permanent. Suppose that $t>s^{*}$ and $\Delta_{\eta, 0}(W, x) \uparrow[t]$. Find some $M$-expansionary stage $s>t$ such that $l(M)[s]$ is large enough so that there is some $y \in \alpha^{[\eta]}$ such that $t<y<l(M)[s]$. Now if $\eta$ didn't define $\Delta_{\eta, 0}(x)$ between $t$ and $s$, then $y$ is a suitable chit (because $\eta$ did not define any $\Delta_{\eta, 0}$ computations between $t$ and $s$ ), thus $\eta$ would define $\Delta_{\eta, 0}(x)$ at $s$.

Claim 2.27. Say $j \in[1, n]$. If there are unboundedly many chits which are eventually $j-1$-suitable (and never cancelled), then $\operatorname{dom} \Delta_{\eta, j}(W)=\alpha$.

Proof. This is like the previous claim. Letting $x, s^{*}, t$ be as above, we find an expansionary stage $s>t$ such that at $s$, there is a chit $y$ which is already $j-1$ suitable and is never cancelled, such that $t<y<l(M)[s]$; this is possible by the assumption that there are unboundedly many such $y$.

Claim 2.28. Suppose that at stage $s, y$ is an active chit for a failed $\Delta_{\eta, j}$ computation. Suppose that $\rho \subset \eta$ works for $T_{j}$. Then $\Gamma_{\rho}$ clears $y$ at $s$.

Proof. We show that if $\Gamma_{\rho}\left(G_{j} \oplus P, y\right) \downarrow[s]$ then this computation must have been defined after the stage $t<s$ at which the chit was originally picked as a chit for a computation $\Delta_{\eta, j}(x)$. Obviously (by the size requirement for $y$ ) we have $x \leqslant y$. As the computation is failed at $s, x$ enters $G_{j}$ at a stage $u \in(t, s)$; 
now $x \leqslant y \leqslant \gamma_{\rho}\left(G_{j} \oplus P, y\right)[u]$ (if $\Gamma_{\rho}\left(G_{j} \oplus P, y\right) \downarrow[u]$ ), destroying the latter computation.

Lemma 2.29. There cannot be unboundedly many chits which are eventually $n$ suitable and which are never cancelled.

Proof. We show that if there are unboundedly many such chits then $\eta$ declares victory after $r^{*}$.

By the fairness assumption, there is some $\beta<\alpha$ such that

$$
C_{0}=\left\{s>r^{*},: \operatorname{Rest}(\eta)[s] \leqslant \beta \quad \& \quad \eta \in \delta[s]\right\}
$$

contains a recursive club. Further, we know that

$$
C_{1}=\left\{s>r^{*}: r(\eta)[s]=0\right\}
$$

is a recursive club. Let $C=C_{0} \cap C_{1} ; C$ contains a recursive club.

By assumption we can find $y>\beta$ and a stage $s \in C$ such that at $s, y$ is an active chit for a failed $\Delta_{\eta, n}$ computation and such that $y<l(M)[s]$. Then $y$ is victorious at $s$, and at $s, \eta$ may win by enumerating $y$ into $Q$.

The following concludes the verifications. For $A, B \subset \alpha$, we say that $A={ }_{*} B$ if there is some $\beta<\alpha$ such that $A \uparrow(\beta, \alpha)=B \uparrow(\beta, \alpha)$. A corollary of the next and previous lemmas is that there is some $j \leqslant n$ such that $\Delta_{\eta, j}(W)={ }_{*} G_{j}$. As $W$ is admissible and $G_{j}$ amenable we get $G_{j} \leqslant{ }_{\alpha} W$.

Lemma 2.30. Suppose that $j \leqslant n$ and $\operatorname{dom} \Delta_{\eta, j}(W)=\alpha$ but $\Delta_{\eta, j}(W) \neq_{*} G_{j}$. Then there are unboundedly many chits which are eventually $j$-suitable and are never cancelled. 
Proof. Much of the proof goes along classical lines. Fix $\beta<\alpha$. Let the functional $\Xi$ converge at $t>r^{*}$ with use $\rho \oplus \pi \subset R \oplus P[t]$ if there is some $\sigma \subset W[t]$ such that $\sigma \subset \Theta(\rho)[t]$ and there is some $y>\beta$ such that at $t,(y, \pi)$ is an active chit for a failed $\Delta_{\eta, j}$ computation with use $\sigma$. If $\Xi$ converges unboundedly often, the success of $K_{\Xi}$ would show that there is some $j$-suitable chit which is never cancelled and which is greater than $\beta$ (protection of $\rho \oplus \pi \subset R \oplus P$ ensures that $\sigma \subset W$ ).

Suppose that $t^{*}>r^{*}$ is any stage. Take some $x>\beta, t^{*}$ such that $\Delta_{\eta, j}(W, x) \neq$ $Q(x)$. Suppose that the correct $\Delta_{\eta, j}(x)$ computation is defined at stage $s>t^{*}$ with associated chit $(y, \pi)$. Let $t>s$ be the stage at which this computation fails; some agent $\rho$ enumerates $x$ into $G_{j}$ at $t$. The standard argument shows that $\eta \subset \rho$ : $\rho$ cannot be stronger than $\eta$ as $t>r^{*}$, and $\eta$ cannot lie to the right of $\rho$ since $\eta$ initializes nodes to its right at $s$ and $s>x$. This argument shows that $x$ is a follower for $\rho$ at $s$ and so that $\rho$ initializes weaker requirements on $\eta$ 's behalf at $s$.

Let $u$ be the least stage greater than $t$ at which $r(\eta)=0$. The key is noticing that $u$ is $M$-expansionary, as it cannot be a limit of $M$-expansionary stages. Thus $y<l(M)[u]$.

We verify that $\pi \subset P$ is preserved until $u$. As mentioned, $\rho$ initializes weaker agents at s. $\rho$ is not initialized until at least after $t$, as $x$ is still a follower at $t$. Thus $\pi \subset P$ is preserved between $s$ and $t$ by $\rho$ 's action. Between $t$ and $u$, restraint which is imposed by $\eta$ protects $\pi \subset P$; recall that $s$ is an $M$-expansionary stage.

Now at $u, y$ is $M$-confirmed so $\sigma \subset \Theta(R)[u]$ (note that $\sigma \subset W$ is always true since we picked the correct $\Delta_{\eta, j}(W)$ computation). Thus $\Xi(R \oplus P) \downarrow[u]$. 


\subsection{Various Constructions}

Here we apply the template to particular classes of admissible ordinals $\alpha$. We recall that we need to describe the tree of agents, assign requirements, define $\delta[s]$, and make sure that $n(\rho)<\omega$ for all $\rho$ and that the fairness assumption holds.

\subsection{1 $\varrho_{\alpha}=\omega$}

Suppose that $\varrho_{\alpha}=\omega$. Fix a partial recursive, 1-1 and onto $p: \omega \rightarrow \alpha$.

The biggest obvious difference between the classical construction and this one is the number of requirements. Initially, we arrange the requirements effectively in order-type $\alpha$. We then use the map $p$ in order to re-arrange the requirements in order-type $\omega$. The tree of agents is thus $\omega$, and at stage $s$, agent $m$ is assigned the requirement $p(m)[s]$; if $p(m) \uparrow[s]$ then $m$ is not assigned any requirement at stage $s$. We let $\delta[s]=\omega$ for all $s$. As every agent is preceded by only finitely many agents, $n(m)[s]<\omega$ for all $m$ and $s$.

The fairness assumption is not hard to verify. Each agent is assigned a requirement at most once. By induction we show for every agent $m$ that init $(m)[\alpha]<\alpha$ and that eventually $m$ does not initialize weaker agents. The first holds by induction, because agent $m$ is initialized only when it is assigned a requirement or when stronger agents initialize it. The second holds because of lemma 2.11 and the fact that for every $m$ there are only finitely many agents $k<m$.

The fact that $\alpha>\omega$ and as far as we can effectively tell, is a regular cardinal, allows for some simplifications in comparison with the classical construction: we no longer require a tree of outcomes to guess the true restraint on a particular agent. For all $k, r(k)$ is constant on a recursive club $C_{k}$ (claim 2.23) and so $\operatorname{Rest}(m)$ is 
bounded on $\cap_{k<m} C_{k}$, which is a recursive club.

[We notice that the price to pay for this simplification is that the restraint has to fall back on limits of expansionary stages, which may be not expansionary themselves; thus in theory a requirement might wish to impose further restraint at such a limit stage, and dropping the restraint might damage the success of the requirement. We noticed however that this does not happen in our construction, as the responsibility to act often lies with the lowness requirements associated with specific tasks (which are of a simpler, finitary nature); the minimality $(M)$ requirements only need to act at successor expansionary stages, so dropping the restraint at limits of expansionary stages does not harm their success.]

\section{A Remark on the Case $\alpha=\omega_{1}^{C K}$}

As mentioned in section 2.1, if $\alpha=\omega_{1}^{C K}$ then Kleene's $\mathcal{O}$ is not $\alpha$-recursive, so the theorems proved do not imply that models with Kleene's $\mathcal{O}$ can be coded. However, $\mathcal{O}$ is $\omega_{1}^{C K}$-r.e., and so can be recursively approximated. We can modify both theorems 2.3 and 2.4 to make $\preccurlyeq$ recursively approximated in a $\Delta_{2}$-fashion, rather than $\alpha$-recursive. There are only minor changes to the construction. A requirement $N_{i, j, \Psi}$ may change its mind about its own necessity, but eventually it guesses correctly. As far as a $D$ - or an $N$-requirement $\eta$ initializing on the behalf of a stronger $M$-requirement $\rho$, to avoid confusion, we let the former initialize whenever the latter define a $\Delta_{\rho, j}(x)$ computation when $x$ is a follower for $\eta$, no matter to which set it is targeted. It is easy to see this doesn't affect fairness of the construction. Finally, the positive ordering requirements $G_{j} \leqslant_{\alpha} G_{i} \oplus L$ for $j \preccurlyeq i$, also may change their mind, but eventually, every number put into $j$ is put into $L$ or $G_{i}$ and the reduction still holds. 
The full details can be found in [GSS].

\subsection{2 $\varrho_{\alpha}^{2}=\omega$ but $\varrho_{\alpha}>\omega$}

The main hurdle in this case is of course that we cannot effectively put the requirements in a list of length $\omega$; we can only effectively approximate such a list. The problem with the approximation is that an agent which is not assigned any requirement may guess unboundedly often that it is assigned some requirement; under the previous rules, each such time would be destructive to weaker agents. [Thus if the $\Delta_{2}$-projectum of $\alpha$ is $\omega$ then the previous construction can be performed without difficulty.]

To overcome this difficulty, we let the tree of agents be $2^{<\omega}$. Each agent guesses whether it, and the agents below it, would be really assigned a requirement or not; agents whose guess is correct will perform their duty successfully.

We noticed that a pure tree construction interferes with the finitary-type requirements, which demand immediate access when they discover a computation they wish to protect, or a follower that is permitted; they cannot wait. We thus rigged the construction in their favor by introducing the first phase of each stage, at which agents working for finitary requirements are allowed to elbow their way to the front of the line. As we shall shortly see, this implies that in order to prove fairness, we need to use the assumption that $\varrho_{\alpha}>\omega$. Thus the arguments for the case $\varrho_{\alpha}=\omega$ are actually needed independently.

We need to approximate a partial, onto map $w \rightarrow \alpha$; for this we need a notion of approximation of a partial function. We use the following:

Definition. Let $\beta \leqslant \alpha$ and $f: \beta \rightarrow \alpha$ be partial. A function $f(x)[s]$ is a strong approximation of $f$ if: 
1. For every $x \in \operatorname{dom} f, f(x)[s]=f(x)$ on all $s$ but an initial segment of $\alpha$.

2. For every $x \notin \operatorname{dom} f, f(x)[s]=0$ for unboundedly many stages $s$.

3. For all $x$, the set of stages $s$ at which $f(x)[s]=0$ is closed.

The approximation is tame if for all $\gamma<\beta$, (1-3) hold at once for all $x<\gamma$. Namely: for all $\gamma<\beta$ there is some $s^{*}$ such that:

a. For all $x \in \gamma \cap \operatorname{dom} f$ and $s>s^{*}, f(x)[s]=f(x)$.

b. $K_{\gamma}=\{s: f(x)[s]=0$ for all $x \in \gamma \backslash \operatorname{dom} f\}$ is a club.

[It follows that if $f$ has a recursive tame strong approximation, then for all $\gamma<\beta$, $\gamma \cap \operatorname{dom} f$ and $f \uparrow(\gamma \cap \operatorname{dom} f)$ are $\alpha$-finite. If $f$ has a recursive strong approximation then we could in fact, in (b), only require that the set $K_{\gamma}$ be merely unbounded, rather than a club; it would still follow that $\gamma \cap \operatorname{dom} f$ is $\alpha$-finite, and then $K_{\gamma}$ would be a uniform intersection of recursive clubs (the stages $s>s^{*}$ at which $f(x)[s]=0$ for any particular $x \notin \operatorname{dom} f)$ and so a club. $]$

If $\operatorname{dom} f=\omega$ then any recursive strong approximation of $f$ is also tame; this is because a finite intersection of recursive clubs is a club.

Proposition 2.31. For every admissible $\alpha$ and $\beta \leqslant \alpha$, every $\Sigma_{2}\left(J_{\alpha}\right) f: \beta \rightarrow \alpha$ has a recursive strong approximation.

Proof. We approximate $f$ in a similar way to that of lemma B.1. Let $\Phi$ be a nice, weak functional such that $\Phi\left(0^{\prime}\right)=f$. For any $x<\beta$ and $s<\alpha$, if there is some $s^{*}<s$ and some $i$ such that for all $t \in\left[s^{*}, s\right]$ we have $\Phi\left(0^{\prime}, x\right)[s]=i$ then let $f(x)[s]=i$. Otherwise, let $f(x)[s]=0$. 
Fix $p: \omega \rightarrow \alpha$ which is partial, $\Sigma_{2}\left(J_{\alpha}\right), 1-1$ and onto. Get a recursive, strong approximation $p[s] . \delta[s] \in 2^{\omega}$ is defined by $\delta[s]=1$ iff $p(n)[s]>0$.

As usual, all requirements are arranged effectively in a list of length $\alpha$. Call an agent $\eta$ active if $\eta(|\eta|-1)=1$. An active agent $\eta$ is assigned to requirement $p(|\eta|-1)[s]$ if the latter converges at $s$. Passive (that is, non-active) nodes are never assigned requirements. Again since every agent is preceded by finitely many agents, $n(\rho)[s]<\omega$ for all $\rho$ and $s$.

The true path is, of course, $\operatorname{dom} p$. We let, for $m<\omega$,

$$
C_{m}=\{s: \operatorname{dom} p\lceil m \subset \delta[s]\}
$$

Since $p[s] \rightarrow p$ is a tame strong approximation, we know that $C_{m}$ contains a club.

By induction we can show that for every $m, \operatorname{init}(\operatorname{dom} p\lceil m)[\alpha]<\alpha$ and that $\operatorname{dom} p\lceil m$ does not initialize weaker requirements. Assume up to $m$. If $\operatorname{dom} p\lceil m$ is passive then it is never assigned any requirement. Otherwise $p(m) \downarrow$ correctly by some stage, and after that stage $\operatorname{dom} p \uparrow m$ will not be initialized because of a reassignment of its requirement. By induction there is a stage after which no $\rho \subset \operatorname{dom} p\lceil m$ initialized $\operatorname{dom} p\lceil m$.

In both cases we know that there is some stage $s_{0}$ after which no $\rho$ which lies to the left of $\operatorname{dom} p\lceil m$ is accessible. Let $K$ be the set of agents which lie to the left of $\operatorname{dom} p\left\lceil m\right.$ that act after stage $s_{0}$. This is an r.e. subset of $2^{<\omega}$. Of course $\left|2^{<\omega}\right|^{J_{\alpha}}=\omega$, and $\varrho>\omega$, so $K$ is $\alpha$-finite. Every $\rho \in K$ acts exactly once after $s_{0}$. The function taking $\rho \in K$ to the stage at which it acts is recursive, hence bounded. Thus eventually $\operatorname{dom} p\lceil m$ is not initialized by nodes to its right, and we have $\operatorname{init}(\operatorname{dom} p\lceil m)[\alpha]<\alpha$.

There are only finitely many $\rho \subset \operatorname{dom} p \uparrow m$, so lemma 2.11 shows that even- 
tually $\operatorname{dom} p\lceil m$ stops initializing nodes extending it.

By claim 2.23, for every $k$ there is a recursive club $D_{k}$ on which $r(\operatorname{dom} p\lceil k)$ is bounded. It follows that on the club $\cap_{k<m} D_{k} \cap C_{m}$, dom $p\lceil m$ is accessible and $\operatorname{Rest}(\operatorname{dom} p\lceil m)$ is bounded.

\subsection{3 $\alpha$ is $\Sigma_{2}$-admissible, but $\operatorname{cf}_{\Sigma_{3}\left(J_{\alpha}\right)}(\alpha)=\omega$}

To enable us to handle trees of height greater than $\omega$, we assume $\Sigma_{2}$-admissibility.

Let $\lambda=\varrho_{\alpha}^{2}$.

Claim 2.32. Let $g: \lambda \rightarrow \alpha$ be $\Sigma_{2}\left(J_{\alpha}\right)$ and partial. Then for all $\beta<\lambda, g\lceil\beta$ is $\alpha$-finite.

Proof. The structure $M=\left(J_{\alpha}, \in, 0^{\prime}\right)$ is admissible. $\lambda=\varrho_{M}$ and $g$ is $M$-partial recursive. For every $\beta<\lambda, \beta \cap \operatorname{dom} g$ is $M$-r.e. and so $\alpha$-finite, and so $g \uparrow \beta$ is $\Delta_{1}(M)$ and so $\alpha$-finite.

It follows that every such $g$ has a recursive, tame strong approximation. It has a recursive strong approximation by proposition 2.31. Further, for every $\beta<\lambda$, the function taking $x \in \beta \cap \operatorname{dom} g$ to the least $s$ such that $g(x)[t]$ is constant on $[s, \alpha)$ is $\Pi_{1}\left(J_{\alpha}\right)$ on an $\alpha$-finite set, hence bounded. Also, as $\beta \backslash \operatorname{dom} g$ is $\alpha$-finite,

$$
\{s<\alpha: \text { for all } x \in \beta \backslash \operatorname{dom} g, g(x)[s]=0\}
$$

is an $\alpha$-finite intersection of recursive clubs, hence is a club. Thus every recursive, strong approximation of $g$ is tame.

$\Sigma_{3}\left(J_{\alpha}\right)$ functions will not have strong approximations; we need a notion of a weak approximation. 
Definition. Let $\beta \leqslant \alpha$ and $f: \beta \rightarrow \alpha$ be total. $f: \beta \times \alpha \rightarrow \alpha$ is a weak approximation of $f$ if for all $x<\beta$,

1. $\{s: f(x)[s]=f(x)\}$ is unbounded.

2. $f(x)[s] \geqslant f(x)$ except for $s$ in an initial segment of $\alpha$.

3. For all limit stages $t, f(x)[t] \leqslant \liminf _{s \rightarrow t} f(x)[s]$.

It follows that for all $x$ for some $s^{*}<\alpha,\left\{s>s^{*}: f(x)[s]=f(x)\right\}$ is a club.

Again, a tame, weak approximation is an approximation for which (1-3) hold simultaneously for all $\gamma<\beta$. Namely, for all $\gamma<\beta$ there is some $s^{*}<\alpha$ such that for all $x<\gamma$ and $s>s^{*}, f(x)[s] \geqslant f(x)$ and $\{s: f(x)[s]=f(x)$ for all $x<\gamma\}$ contains a club. If $\operatorname{dom} f=\omega$ then any weak approximation is also tame.

Let $p: \lambda \rightarrow \alpha$ be partial, $\Sigma_{2}\left(J_{\alpha}\right), 1-1$ and onto, and let $p[s]$ be a recursive, tame, strong approximation to $p$.

Claim 2.33. $\operatorname{cf}_{\Sigma_{3}\left(J_{\alpha}\right)}(\lambda)=\omega$.

Proof. Let $f: \omega \rightarrow \alpha$ be $\Sigma_{3}\left(J_{\alpha}\right)$ and cofinal. Consider $p^{-1} \circ f: \omega \rightarrow \lambda$; it is a $\Sigma_{3}\left(J_{\alpha}\right)$ function. Let $\beta=$ sup range $p^{-1} \circ f$. If $\beta<\lambda$ then $p$ " $\beta$ is bounded below $\alpha$, but $p$ " $\beta$ contains range $f$.

Lemma 2.34. There is an increasing and cofinal $f: \omega \rightarrow \lambda$ that has a recursive, weak approximation $f[s]$ such that each $f[s]$ is increasing.

Proof. Fix $g: \omega \rightarrow \lambda$ cofinal such that

$$
g(n)=\beta \Leftrightarrow \exists x \forall y \exists z R(n, \beta, x, y, z)
$$


where $R$ is recursive. For simplicity assume that always $x>0$. Let the length of agreement be

$$
l(n, \beta, x)[s]=\min \{y<s: \neg \exists x R(n, \beta, x, y, z)\} .
$$

Use the standard pairing function $i \rightarrow\left((i)_{0},(i)_{1}\right) ; \lambda$ is closed under this pairing.

Let $f_{0}(n)[s]$ be the least $i<\lambda$ such that letting $\beta=(i)_{0}$ and $x=p\left((i)_{1}\right)[s]>0$, for all $t<s, l(n, \beta, x)[t]<l(n, \beta, x)[s]$ (if no such pair exists let $g_{0}(n)[s]=s$.)

Fix $n<\omega$. Let $i(n)$ be the least $i<\lambda$ such that for $\beta=(i)_{0}(=g(n))$ and $x=p\left((i)_{1}\right)$, for all $y<\alpha$ there is some $z<\alpha$ such that $R(n, \beta, x, y, z)$.

Claim 2.35. $\lim _{s \rightarrow \alpha} l(n, \beta, x)[s]=\alpha$.

Proof. By admissibility; the function taking $y<\alpha$ to the least $z$ such that $R(n, \beta, x, y, z)$ is recursive and so bounded on every $y<\alpha$.

Let $K_{n}=\left\{i<i(n):(i)_{1} \in \operatorname{dom} p\right\} ; K_{n}$ is $\alpha$-finite. For all $i \in K_{n}$ there is some $y$ such that for no $z$ do we have $R\left(n,(i)_{0}, p\left((i)_{1}\right), y, z\right)$. Let $y_{i}$ be the least such $y . i \rightarrow y_{i}$ is a $\Sigma_{2}\left(J_{\alpha}\right)$ function (as $p \uparrow K_{n}$ is $\alpha$-finite) and so is bounded. It follows that from some stage, $f_{0}(n)[s] \geqslant i(n) \geqslant g(n)$, and that $f_{0}(n)[s]=i(n)$ for unboundedly many $s$. Also, $i: \omega \rightarrow \lambda$ is cofinal.

We now define $f_{1}(n)[s]$ by induction on $s$. In general, we let $f_{1}(n)[s]$ equal $f_{0}(n)[s]$, unless $s$ is limit and $\liminf _{t \rightarrow s} f_{1}(n)[t]<f_{1}(n)[s]$; in that case we let $f_{1}(n)[s]=\liminf _{t \rightarrow s} f_{1}(n)[t]$. Suppose that after $s_{n}, f_{0}(n)[s] \geqslant i(n)$; and let $C_{n}$ be the set of limit points of $\left\{s>s_{n}: f_{0}(n)[s]=i(n)\right\} . C_{n}$ is a club and $f_{1}(n)[s]=i(n)$ if $s \in C_{n}$. Also, $f_{1}(n)[s] \geqslant i(n)$ for all $s>s_{n}$. Thus $f_{1}[s]$ is a weak approximation of $i$.

Finally, let $f_{2}(n)[s]=\max \left\{f_{1}(k): k \leqslant n\right\}$. Then $f_{2}[s]$ is a weak approximation of $f(n)=\max \{i(k): k \leqslant n\}$ and is as required. 
We now describe our constructions. The tree of agents is the subtree of $(\alpha \cup$ $\{$ yes, no $\})^{<\lambda}$ of nodes $\eta$ satisfying the following:

1. For every even ordinal $i<\operatorname{dom} \eta, \eta(i) \in \alpha$, and for every odd ordinal $i<$ $\operatorname{dom} \eta, \eta(i) \in\{$ yes, no $\}$.

2. The set $\{i<\operatorname{dom} \eta: \eta(i)=$ yes $\}$ is finite.

First, all requirements (except for the $T_{n} \mathrm{~s}$ ) are placed in an effective list of length $\alpha$. In place 0 we place no requirement.

An agent $\eta$ of limit length works for no requirement. An agent $\eta$ of length $\beta+1$ works for $\eta(\beta)$, if $\beta$ is even. If $\beta$ is odd and $\eta(\beta)=$ no then $\eta$ works for no requirement. If $\beta$ is odd and $\eta(\beta)=$ yes then $\eta$ works for $T_{n}$, where $n=$ $\mid\{i<\beta: \eta(i)=$ yes $\} \mid$. These assignments are fixed throughout the construction, so no initializations are made on account of agents discovering they are working for a new requirement. The restriction on agents exactly implies that for all $\eta$, $n(\eta)<\omega$.

The nodes are ordered lexicographically; yes is stronger than no.

At stage $s$, we define the path of accessible nodes. Let

$$
\delta_{0}(i)[s]= \begin{cases}p(\beta)[s] & \text { if } i=2 \beta<\lambda, \\ \text { yes } & \text { if } i=2 \beta+1<\lambda \text { and } \beta \in \text { range } f[s], \\ \text { no } & \text { if } i=2 \beta+1<\lambda \text { and } \beta \notin \text { range } f[s] .\end{cases}
$$

Let $\delta[s]$ be the maximal $\eta \subset \delta_{0}[s]$ such that for all $i<\operatorname{dom} \eta, \eta \uparrow i$ is a node on the tree of agents. 
The true path $\delta$ is defined in a similar fashion to $\delta_{0}[s]$ :

$$
\delta(i)= \begin{cases}p(\beta) & \text { if } i=2 \beta<\lambda \text { and } \beta \in \operatorname{dom} p, \\ 0 & \text { if } i=2 \beta<\lambda \text { and } \beta \notin \operatorname{dom} p, \\ \text { yes } & \text { if } i=2 \beta+1<\lambda \text { and } \beta \in \text { range } f, \\ \text { no } & \text { if } i=2 \beta+1<\lambda \text { and } \beta \notin \text { range } f .\end{cases}
$$

Lemma 2.36. For every $i<\lambda, \delta \uparrow i$ is a node on the tree of agents. For every $i<\lambda, \delta \uparrow i \subset \delta[s]$ for club many $s$, and there is some $s_{i}<\alpha$ after which no $\eta<_{\ell} \delta \uparrow i$ is accessible.

Proof. For every $\beta<\lambda, p \uparrow \beta$ is $\alpha$-finite; $f \uparrow f^{-1} \beta$ is finite and so $\alpha$-finite; this implies that $\delta \uparrow 2 \beta$ is $\alpha$-finite. It mentions only finitely many yes answers so it is a node on the tree of agents.

The second and third statements follow from the fact that $p[s] \rightarrow p$ is a tame, strong approximation and that $f[s] \rightarrow f$ is a tame, weak approximation. Fix $\beta<\lambda$. Let $n_{\beta}$ be the least $n$ such that $f(n)>\beta$. Let $s_{\beta}$ be a stage such that:

- For all $\gamma \in \beta \cap \operatorname{dom} p$ and $s>s_{\beta}, p(\gamma)[s]=p(\gamma)$.

- For all $k \leqslant n_{\beta}$ and all $s>s^{*}, f(k)[s] \geqslant f(k)$.

- The set

$$
C_{\beta}=\left\{s>s_{\beta}: \begin{array}{l}
\forall \gamma \in \beta \backslash \operatorname{dom} p, p(\gamma)[s]=0 \quad \& \\
\\
\quad \text { for all } k \leqslant n_{\beta}, f(k)[s]=f(k)
\end{array}\right\}
$$

is a (recursive) club.

If $s \in C_{\beta}$ then $\delta \uparrow 2 \beta \subset \delta[s]$. Also, no $\eta<_{\ell} \delta \uparrow 2 \beta$ can be accessible after $s_{\beta}$ : say $s>s_{\beta}$ and $\delta \uparrow 2 \beta \not \subset \delta[s]$; let $\rho=\delta \cap \delta[s]$ and let $i=\operatorname{dom} \rho$. If $i=2 \beta$ then since

$$
\delta[s](i)=p(\beta)[s] \geqslant p(\beta)=\delta(i)
$$


we have $\delta<_{\ell} \delta[s]$. Suppose that $i=2 \beta+1 . \rho \subset \delta, \delta[s]$ implies that range $f \cap \beta=$ range $f[s] \cap \beta$. If $f(n)[s]=\beta$ then $f(n) \leqslant f(n)[s]$ must also equal $\beta$. So we must have $f(n)[s]>\beta=f(n)$ so $\delta(i)=$ yes and $\delta[s](i)=$ no and again $\delta<_{\ell} \delta[s]$.

We want to show that the fairness assumption holds. As usual, we first show that for all $\eta$ on the true path, init $(\eta)[\alpha]<\alpha$ and that $\eta$ eventually stops initializing all weaker nodes. Assume $\eta \subset \delta$ and that this holds for all $\rho \subsetneq \eta$.

Say that after $s_{\eta}$, no node to the left of $\eta$ is ever accessible. The set of agents to the left of $\eta$ ever accessible is $\alpha$ finite, and each acts at most once after $s_{\eta}$. $\varrho_{\alpha}=\alpha$ and so the set of such nodes that ever act after $s_{\eta}$ is $\alpha$-finite. It follows that after some stage, $\eta$ does not get initialized by some node to its left. By the induction assumption, we have $\operatorname{init}(\eta)[\alpha]<\alpha$.

To show that after some stage, $\eta$ does not initialize weaker agents, it is enough, by lemma 2.11 , to show that if $\eta$ works for a $D, N$ or a $Z$ requirement, then $\eta$ eventually stops initializing on behalf of agents $\rho \subsetneq \eta$. We know that for each $\rho \subsetneq \eta$ there is a stage after which $\eta$ does not initialize on $\rho$ 's behalf; call the least such stage $t_{\rho} . \rho \rightarrow t_{\rho}$ is $\Pi_{1}\left(J_{\alpha}\right)$ on $\operatorname{dom} \eta$ and so bounded, as required.

Finally, we need to show that for all $\eta \subset \delta$, Rest $(\eta)[s]$ is bounded on a recursive club. In fact, we know that for all $\rho \subsetneq \eta, r(\rho)[s]$ is either eventually constant or 0 on a recursive club. The set $K$ of $\rho \subsetneq \eta$ such that $r(\rho)[s]$ is eventually constant is $\Sigma_{2}\left(J_{\alpha}\right)$, hence $\alpha$-finite. The function taking $\rho \in K$ to that constant value is also $\Sigma_{2}\left(J_{\alpha}\right)$, hence $\alpha$-finite (and bounded); so is the function taking $\rho \in K$ to the least stage $u_{\rho}$ from which $r(\rho)$ is constant.

Let, for $\rho \in K, D_{\rho}=\alpha \backslash u_{\rho}$, and for other $\rho \subsetneq \eta, D_{\rho}=\{s: r(\rho)[s]=0\}$. $D=\cap_{\rho \subsetneq \eta} D_{\rho}$ is a club on which $\operatorname{Rest}(\eta)[s]$ is bounded. 


\section{CHAPTER 3}

\section{CODING EFFECTIVE SUCCESSOR MODELS}

In this chapter we combine the construction of effective successor models (see [NSS98, Thm. 6.1]) with the tree techniques of section 2.3.3 and add the construction of an exact pair to code an effective model of arithmetic into the $\alpha$-r.e. degrees, when $\alpha$ is $\Sigma_{2}$-admissible but $\operatorname{cf}_{\Sigma_{3}\left(J_{\alpha}\right)}(\alpha)=\omega$. This contrasts with the results of chapter 4 .

We first define the notions used. We modify the definition of an SW set to adjust to working above a bottom degree $\mathbf{b}$.

Definition. The $S W$ set defined by a quintuple of parameters $\overline{\mathbf{p}}=(\mathbf{b}, \mathbf{p}, \mathbf{q}, \mathbf{r}, \mathbf{l})$ (denoted by $\left.G_{\overline{\mathbf{p}}}\right)$ is the collection of $\mathbf{g} \in(\mathbf{b}, \mathbf{r})$ which are minimal solutions above $\mathbf{b}$ of the inequality $\mathbf{g} \vee \mathbf{p} \geqslant \mathbf{q}$. For $\mathbf{g}_{0}, \mathbf{g}_{1} \in G_{\overline{\mathbf{p}}}$, we let $\mathbf{g}_{0} \leqslant \overline{\mathbf{p}} \mathbf{g}_{1}$ if $\mathbf{g}_{0} \leqslant \mathbf{g}_{1} \vee \mathbf{l}$.

Next, the definition of the correctness condition $\chi_{\mathrm{sw}}$ and the associated notions which define a model of Robinson arithmetic coded by $\leqslant_{\overline{\mathbf{p}}}$ are taken from the beginning of chapter 2 verbatim, but with $G_{\overline{\mathbf{p}}}$ according to the new definition (working above $\mathbf{b}$ ).

Definition. Let $\overline{\mathbf{p}}$ be a quintuple satisfying $\chi_{\mathrm{Sw}}$. We say that $M_{\overline{\mathbf{p}}}$ is an effective successor model if the following two conditions hold.

1. There is some quadruple $\overline{\mathbf{e}}=\left(\mathbf{e}_{0}, \mathbf{e}_{1}, \mathbf{f}_{0}, \mathbf{f}_{1}\right)$ such that for all $\mathbf{x} \in M_{\overline{\mathbf{p}}}$, if $(\mathbf{x}>0)^{M_{\overline{\mathbf{p}}}}, i<2$ and $M_{\overline{\mathbf{p}}} \models " \mathbf{x}=i \bmod 2 "$, then

$$
\mathbf{x}=\left(\mathbf{e}_{i} \vee(\mathbf{x}-1)^{M_{\overline{\mathbf{p}}}}\right) \wedge \mathbf{f}_{i}
$$

2. For every $\mathrm{x} \in M_{\overline{\mathbf{p}}}$, the set

$$
\left\{\mathbf{y} \in M_{\overline{\mathbf{p}}}: \mathbf{y}<^{M_{\overline{\mathbf{p}}}} \mathbf{x}\right\}
$$


has a least upper bound (in $\mathcal{R}_{\alpha}$ ) which we denote by $\sum_{\overline{\mathbf{p}}} \mathbf{x}$. Further, for all $\mathbf{y} \in M_{\overline{\mathbf{p}}}$ such that $\mathbf{y} \geqslant^{M_{\overline{\mathbf{p}}}} \mathbf{x}$ we have $\mathbf{y} \nless \sum_{\overline{\mathbf{p}}} \mathbf{x}$.

We let $\chi_{\text {effective }}(\overline{\mathbf{p}}, \overline{\mathbf{e}})$ state that $\overline{\mathbf{p}}$ satisfies $\chi_{\text {sw }}$ and that $\overline{\mathbf{e}}$ witnesses that $M_{\overline{\mathbf{p}}}$ is an effective successor model. Note that $\chi_{\text {effective }}$ can indeed be stated in a first-order fashion.

Also, we note that if $\overline{\mathbf{p}}$ satisfies $\chi_{\mathrm{Sw}}, M_{\overline{\mathbf{p}}}$ is standard and the elements of $M_{\overline{\mathbf{p}}}$ form an independent set, then (2) of the definition holds automatically, so (1) is really the interesting requirement for effectiveness of the model, as far as constructions go. The second condition will be used in chapter 4 .

We also recall that if $\mathcal{H}$ is an independent set of degrees, then an exact pair for $\mathcal{H}$ is a pair of degrees $\mathbf{c}_{0}, \mathbf{c}_{1}$ such that the intersection of the principal ideals determined by $\mathbf{c}_{0}$ and $\mathbf{c}_{1}$ is exactly the ideal generated by $\mathcal{H}$; namely, for all $\mathbf{x}$,

$$
\mathbf{x} \leqslant \mathbf{c}_{0} \quad \& \quad \mathbf{x} \leqslant \mathbf{c}_{1}
$$

iff there is some finite $\mathcal{F} \subset \mathcal{H}$ such that

$$
\mathbf{x} \leqslant \bigvee \mathcal{F}
$$

In this chapter we prove:

Theorem 3.1. Suppose that $\alpha$ is $\Sigma_{2}$-admissible and that $\operatorname{cf}_{\Sigma_{3}\left(J_{\alpha}\right)}(\alpha)=\omega$. Let $\mathbf{u}$ be a promptly permitting $\alpha$-r.e. degree. Then there is a quintuple $\overline{\mathbf{p}}$, a pair $\mathbf{c}_{0}, \mathbf{c}_{1}$ and a quadruple $\overline{\mathbf{e}}$, all below $\mathbf{u}$, such that $M_{\overline{\mathbf{p}}}$ is a standard effective successor model (witnessed by $\overline{\mathbf{e}}$ ), and such that $\mathbf{c}_{0}, \mathbf{c}_{1}$ form a minimal pair for $M_{\overline{\mathbf{p}}}$.

For the definition of prompt permitting and a discussion of how it is used in our construction, see appendix E. We also remark that the theorem can be proven for 
admissible ordinals $\alpha$ such that $\varrho_{\alpha}^{2}=\omega$; the construction is similar, except that the trees of agents that we use are $\omega$ (if $\varrho_{\alpha}=\omega$ or $2^{<\omega}$ (if $\varrho_{\alpha}^{2}=\omega<\varrho_{\alpha}$ ); assignments of requirements to nodes and the accessible nodes are defined as in sections 2.3.1 and 2.3.2.

\subsection{Construction}

We are given a promptly permitting set $U$. We are also given an $\alpha$-finite partial ordering $\preccurlyeq$ on $\omega$, together with an infinite, $\alpha$-finite set of $\preccurlyeq$-minimal elements $H$ which we enumerate as $\left\{h_{n}: n<\omega\right\}$. We construct sets $B, P, Q, L, E_{0}, E_{1}, C_{0}, C_{1}$ and $G_{n}$ (for $\left.n<\omega\right)$; we require that for all $n<\omega, G_{n}^{[0]}=B$. We denote $G_{h_{n}}$ by $H_{n}$. We let $R=\oplus_{n<\omega} G_{n}, F_{0}=\oplus_{n<\omega} H_{2 n}$ and $F_{1}=\oplus_{n<\omega} H_{2 n+1}$.

The requirements are:

1. $T_{n}: Q \leqslant \alpha G_{n} \oplus P$.

2. $M_{\Theta, \Phi, W}$ : If $\Theta(R)=W$ and $\Phi(B \oplus W \oplus P)=Q$ then there is some $j$ such that $G_{j} \leqslant \alpha B \oplus W$.

3. $D_{j, \Psi}: \Psi\left(\oplus_{i \neq j} G_{i}\right) \neq G_{j}$.

4. $N_{i, j, \Psi}$ : If $j \npreceq i$ then $\Psi\left(G_{i} \oplus L\right) \neq G_{j}$.

5. $K_{\Xi, K}$ : If for all $x \in K$ there are unboundedly many $s$ such that $x \in \Xi(R \oplus$ $P \oplus Q \oplus L)[s]$ then from some $s$ onward, $K \subset \Xi(R \oplus P \oplus Q \oplus L)[s]$ is correct.

6. $Y_{n, \Phi}$ for $n>0$ : Let $i=n \bmod 2$. If $\Phi\left(H_{n-1} \oplus E_{i}\right)=\Phi\left(F_{i}\right)$ is total then $\Phi\left(F_{i}\right) \leqslant \alpha H_{n}$

7. $A_{\Phi}$ : If $\Phi\left(C_{0}\right)=\Phi\left(C_{1}\right)$ is total then for some $n, \Phi\left(C_{0}\right) \leqslant \alpha \oplus_{k \leqslant n} H_{k}$. 
The positive ordering requirements (which are met by permitting and coding techniques), are:

- If $j \preccurlyeq i$ then $G_{j} \leqslant \alpha G_{i} \oplus L$.

- If $n>0$ and $i=n \bmod 2$ then $H_{n} \leqslant \alpha H_{n-1} \oplus E_{i}$.

- All sets constructed are recursive in $U$.

Let $\lambda=\varrho_{\alpha}^{2}$. Let $p: \lambda \rightarrow \alpha$ be partial, $1-1$, onto and $\Sigma_{2}\left(J_{\alpha}\right)$; let $p[s]$ be a recursive, strong, tame approximation to $p$ (see proposition 2.31 and the discussion after claim 2.32). Let $f: \omega \rightarrow \lambda$ be increasing and cofinal, with a recursive weak approximation $f[s]$, such that each $f[s]$ is increasing (see lemma 2.34).

\subsubsection{The Machine}

We use a tree of strategies (a subset of $\alpha^{<\lambda}$ ) which is almost identical to the tree of section 2.3.3; nodes of the tree guess the values of $p$ and $f$. However, we also treat the tree as a pinball machine, where certain nodes act as holes from which balls are released; other nodes act as gates at which balls may reside.

As in section 2.3.3, only nodes which guess only finitely many elements to be in the range of $f$ are considered as agents on the tree. An agent $\eta$ which is responsible for guessing if some number $\epsilon$ is in range $f$, and guesses in the affirmative, is called a pregate. A block consists of the collection of nodes which lie between two pregates. For simplicity, in this construction we let all nodes in each block work on the same kind of requirement; we will need to show that every for requirement $R$ there is indeed a node on the true path working for $R$. Luckily, elementary arithmetic suffices: all we need to do is to require that for all $n<\omega, f(n+1)>f(n) \cdot 2$. This 
will ensure that the blocks on the true path are long enough so that no requirement is missed. We can easily arrange for that, as $\lambda$ is closed under addition.

We only assign tasks to agents of successor length. Let $\eta$ be an agent of length $\beta+1$.

1. If $\beta$ is even then we let $\eta$ guess the range of $f . \eta$ is a pregate if $\eta(\beta)=0$.

Definition. An agent is a node $\rho \in \alpha^{<\lambda}$ such that there are only finitely many pregates $\eta \subset \rho$. If $\rho$ is an agent then we let $n(\rho)$ be the number of pregates properly contained in $\rho$.

If $\eta$ is a pregate then we let $\eta$ work for $T_{n(\eta)}$.

2. Suppose that $\beta$ is odd. Suppose that $(n(\eta))_{0}=\langle X, k\rangle$. We have the following cases:

- If $X=M$ and $\eta(\beta)=(\Theta, \Phi, W)$ then $\eta$ works for $M_{\Theta, \Phi, W}$.

- If $X=K$ and $\eta(\beta)=(\Xi, K)$ then $\eta$ works for $K_{\Xi, K}$.

- If $X=D$ and $\eta(\beta)=\Psi$ then $\eta$ works for $D_{k, \Psi}$.

- If $X=N, k=\langle i, j\rangle$ and $\eta(\beta)=\Psi$ then $\eta$ works for $N_{i, j, \Psi}$ (Note that $i, j \leqslant k \leqslant n(\eta))$.

- If $X=Y$ and $\eta(\beta)=\Phi$ then $\eta$ works for $Y_{k, \Phi}$.

- If $X=A$ and $\eta(\beta)=\Phi$ then $\eta$ works for $A_{\Phi}$.

(We use $(n(\eta))_{0}=\langle X, k\rangle$ (rather than $n(\eta)=\langle X, k\rangle$ ) because we want each $\langle X, k\rangle$ to appear infinitely often on the true path.)

For every node $\eta$, we let $\epsilon(\eta)$ be the ordertype of nodes preceding $\eta$ which work for the same kind of requirement as does $\eta$. To formally define "same kind": 
- All $K$ requirements are of the same kind.

- All $M$ requirements are of the same kind.

- For any $j$, all $D_{j, \Psi}$ requirements are of the same kind.

- For any $i, j$, all $N_{i, j, \Psi}$ requirements are of the same kind.

- For any $n$, all $Y_{n, \Phi}$ requirements are of the same kind.

- All $A$ requirements are of the same kind.

Also, all nodes of odd, non limit length (these are the codes which guess the range of $f$ ) are of the same kind.

$\delta[s]$, the path of accessible nodes, is calculated by induction; the induction halts at a limit level when we reach a node which is not an agent $(n(\eta)=\omega)$. Suppose that $\eta \subset \delta[s]$; we want to find which immediate successor of $\eta$ is also accessible at $s$ (assuming we did not halt the definition of $\delta[s]$ ); there are two cases. Let $\beta=\operatorname{dom} \eta$ and $\epsilon=\epsilon\left(\eta^{\urcorner} 0\right)$.

1. If $\beta$ is even then $\eta^{\frown} 0$ is accessible at $s$ if $\epsilon \in$ range $f[s]$; otherwise, $\eta^{\frown} 1 \subset \delta[s]$ (that is, we define $\delta(\beta)=0[s]$ in the first case, 1 in the other).

2. If $\beta$ is odd then $\eta^{\frown} p(\epsilon)[s] \subset \delta[s]$.

Definition. A $Y$-gate is a pregate $\rho$ such that the nodes in the block which ends with $\rho$ work for a $Y$ requirement (that is, $(n(\rho))_{0}=\langle Y, k\rangle$ for some $k$ ). An A-gate is a pregate such that the nodes in the block which ends with $\rho$ work for an $A$ requirement. 


\section{Restraint}

Let $M=M_{\Theta, \Phi, W}$ be a minimality requirement. A number $y$ is $M$-confirmed at $s$ if

$$
\Phi(B \oplus W \oplus P, y) \downarrow=Q(y)[s]
$$

with use $\zeta \oplus \sigma \oplus \pi=\phi(B \oplus W \oplus P, y)[s]$, and

$$
\sigma \subset \Theta(R)[s]
$$

The length of agreement is

$$
\ell(M)[s]=\max \{z \mid \forall y<z[y \text { is } M \text {-confirmed at } s]\}
$$

We also let

$$
\ell\left(Y_{n, \Phi}\right)[s]=\max \left\{\beta: \Phi\left(H_{n-1} \oplus E_{i} ; \beta\right) \downarrow=\Phi\left(F_{i} ; \beta\right) \downarrow[s]\right\} .
$$

Similarly, we let

$$
\ell\left(A_{\Phi}\right)[s]=\max \left\{\beta: \Phi\left(C_{0} ; \beta\right) \downarrow=\Phi\left(C_{1} ; \beta\right) \downarrow[s]\right\}
$$

Let $\eta$ be an agent which works for requirement $X$ of type $M, Y$ or $A$. A stage $s$ is $\eta$-expansionary if $\eta$ is accessible at $s$ and for all $t<s$ at which $\eta$ was accessible, $\ell(X)[t]<\ell(X)[s]$.

Again suppose that $\eta$ is an agent working for an $M, Y$ or $A$ requirement, and suppose that $\eta$ is accessible at $s$. At $s, \eta$ imposes restraint $r(\eta)$ on weaker nodes. The restraint is 0 if $s$ is $\eta$-expansionary, or is a limit of $\eta$-expansionary stages. Otherwise, we let the restraint be

$$
\sup \{t<s: t \text { is } \eta \text {-expansionary }\} \text {. }
$$


For agent $\eta$, the restraint $\operatorname{Rest}(\eta)[s]$ imposed on $\eta$ at $s$ is

$$
\sup _{\eta^{\prime} \subsetneq \eta} r\left(\eta^{\prime}\right)[s]
$$

(where for an agent $\eta^{\prime}$ working for a requirement which is not $M, Y$ or $A, r\left(\eta^{\prime}\right)=0$ always.) We emphasize that in calculating the restraint on $\eta$ we only consider nodes properly contained in $\eta$ and not other nodes which are stronger than $\eta$ (nodes which lie to $\eta$ 's left); these nodes impose restraint by means of initialization).

For shorthand, we let $\chi[s]: \operatorname{dom} \delta[s] \rightarrow \alpha$ be defined by

$$
\chi(\beta)=r(\delta \uparrow \beta)[s] .
$$

We often say that the pair $\eta, \xi$ is accessible at $s$ if $\eta \subset \delta[s]$ and $\xi \subset \chi[s]$.

\section{Followers and Balls}

A node $\eta$ working for a $D$ or an $N$ requirement attempts to meet the requirement by appointing followers. A follower $x$ for a node $\eta$ working for $D_{j, \Psi}$ is realized at $s$ if $\Psi\left(\oplus_{i \neq j} G_{i}, x\right) \downarrow=0[s]$; a follower $x$ for a node $\eta$ working for $N_{i, j, \Psi}$ is realized at $s$ if $\Psi\left(G_{i} \oplus L, x\right) \downarrow=0[s]$.

As we'll describe shortly, some of these followers will develop long entourages and will trickle down the path of nodes which lie below $\eta$ in that part of the construction which is the pinball machine. To avoid interfering with infimum requirements $(Y, A, M)$, such followers need to guess the restraint imposed by various nodes preceding $\eta$. The classical construction would have the tree do the guess work by adding nodes which guess the outcome $r\left(\eta^{\prime}\right)$ of every agent $\eta^{\prime}$. However, in the setting of admissible recursion theory (even under the assumption of $\Sigma_{2^{-}}$ admissibility), this approach unfortunately produces problems: it would make the true path too complicated so that it may have length less than $\lambda$. We thus need 
to leave the individual guesswork to every follower. If $x$ is a follower for $\eta$, then we let $\xi(x)=\chi[s]\lceil\operatorname{dom} \eta$, where $s$ is the stage at which $x$ is appointed. For any other ball $z$ in $x$ 's entourage (this notion is described shortly) we let $\xi(z)=\xi(x)$.

To get the reducibilities $H_{n} \leqslant{ }_{\alpha} E_{i} \oplus H_{n-1}$, to every ball (number) which is targeted for $H_{n}$, we need to assign a trace which is targeted for $H_{n-1}$ or for $E_{i}$. The resulting string of balls $y_{0}, y_{1}, y_{2}, \ldots, y_{k}$ is called an entourage; $y_{0}$ is the follower and $y_{i+1}$ is $y_{i}$ 's trace. In a sense, the entire entourage, albeit changing in its elements, is the basic unit on the machine. For example, all balls in the same entourage have the same priority (that of the follower), and when a stronger ball or agent receives attention, all balls of the entourage are cancelled (removed from the machine).

There are four possible situations in which an entourage may find itself. Suppose that $\eta$ is an agent which targets its followers for $H_{n}, n>0$.

1. $\eta$ has appointed a follower $x$, which gets a trace $t$ targeted for $E_{i}(i=n$ $\bmod 2$ ). The follower and its trace are waiting at $\eta$.

2. $x$ is realized; the entourage is waiting, in a descending fashion, at gates below $\eta$. In more detail: the entourage $y_{0}, \ldots, y_{k}$ is partitioned into segments $\left(y_{0}, \ldots, y_{l_{1}}\right),\left(y_{l_{1}+1}, \ldots, y_{l_{2}}\right), \ldots,\left(y_{l_{m-1}+1}, \ldots, y_{l_{m}}\right)$; for $j<m$ we let $\bar{y}_{j}=\left(y_{l_{j}+1}, \ldots, y_{l_{j+1}}\right)\left(\right.$ where $\left.l_{0}=-1\right)$. Each segment $\bar{y}_{0}, \ldots, \bar{y}_{m-2}($ which in fact consists of one or two balls) is waiting in a corral which lies just behind a gate $\rho \subset \eta ; \bar{y}_{j+1}$ lies below $\bar{y}_{j}$. The last segment $\bar{y}_{m-1}$ is located at a gate, at the same height at which $\bar{y}_{m-2}$ is waiting, or below it. The last ball of the entourage is targeted either for some $E_{j}$ or for $H_{0}$.

3. The entourage is partitioned as before, and as before all but the last segment wait in corrals in a descending fashion. The last segment is lying at the 
bottom of the machine in the permitting bin. The next to last ball in the entourage is targeted for $H_{0}$ or for some $E_{j}$, and the last ball is targeted for $B$. At most one ball in this last segment is targeted for some $H_{n}$.

4. The entourage is partitioned as before and again all segments but the last lie in corrals in a descending order. The last segment (which again has a last trace targeted for $B$ ) is waiting in some cage which lies behind some pregate $\rho \subset \eta$. It is not necessarily the case that this pregate $\rho$ lies below (or at the same level of) the corral in which the one-before-last segment of $x$ 's entourage lies.

The possible movement balls in an entourage might go through correspond to these positions.

1. When $\eta$ is accessible and $x$ is realized, $x$ and $t$ will be dropped from $\eta$. They arrive at the highest gate $\rho$ below $\eta . x$ is placed in $\rho$ 's corral, and $t$ is placed in the gate.

2. The last segment is located at gate $\rho$, and $\rho, \xi(x) \uparrow \operatorname{dom} \rho$ are accessible. This last segment is released from the gate $\rho$ and falls down to the next gate $\rho^{\prime}$; all but the last ball of the segment are placed in the corral belonging to $\rho^{\prime}$, and the last ball is placed in the permitting bin and is appointed a trace, targeted for $B$. If $\rho$ is the lowest gate below $\eta$, then all the balls which were released are placed in the permitting bin and are assigned a trace targeted for $B$.

3. At some stage, the follower $x$ is permitted by $U$. There are two possibilities:

(a) There is no ball targeted for some $H_{n}$ among the balls in $x$ 's entourage which are waiting in the permitting bin. In this case all balls in the 
permitting bin are enumerated into their target sets. The new last ball of the entourage is now waiting in some corral below $\eta$; it rolls out to the corresponding gate, and is assigned new traces (as will be described shortly).

(b) There is one ball $y$ in $x$ 's entourage, which is waiting in the permitting bin and is targeted for $H_{n} . y$ is enumerated into $C_{0}^{[n]}$, but the ball $y$ is not discarded from the entourage. The last trace (for $B$ ) is enumerated into $B$ (and the ball is discarded). The balls left in the bin receive a new trace for $B$ and are all placed in the cage which lies just behind the unique pregate $\rho \subset \eta$ such that $n(\rho)=n$. [The tracing instructions will show that balls in an entourage associated with hole $\eta$ are only targeted for $H_{k}$ such that $k<n(\eta)$, so such a pregate exists.]

4. $\rho, \xi(x)\lceil\operatorname{dom} \rho$ are accessible. If $x$ is not promptly permitted by $U$ then $x$ and the rest of its entourage are cancelled. If it is, then the balls which were waiting in the cage are released and roll down to the bottom of the machine. The unique ball $y$ which was waiting at $\rho$ 's cage and which is targeted for some $H_{n}$ is enumerated into $C_{1}^{[n]}$ and into $H_{n}$; the rest of the balls are enumerated into their target sets. If the follower $x$ was not just enumerated into its target set, then as in case (3)(a), the new last ball of the entourage rolls out from a corral and is assigned new traces. If $x$ was enumerated then $\eta$ declares victory and cancels all other followers which belong to $\eta$.

Remark 3.2. Every entourage receives attention finitely many times. In particular, the composition of the entourage and the balls' location on the machine are well- 
defined at limit stages.

We said (cases (3)(a) and (4)) that when a new last ball of the entourage rolls from the corral into the gate, it is assigned new traces. The traces are appointed not to interfere with the preservation that is performed by nodes in the block below the gate. Suppose that $\rho$ is a gate and nodes in the block below $\rho$ are working for $Y_{m, \Phi}$; we do not want balls targeted for both $H_{m-1} \oplus E_{j}$ and $F_{j}(j=m \bmod 2)$ to lie at the gate at the same time (unless they are targeted for the intended infimum $\left.H_{m}\right)$. This can always be done. Suppose that a ball $x$, targeted for $H_{n}$, just rolled out to the gate. Let $i=n \bmod 2$.

- If $m=n$ then $\rho$ does not care about $x$; a trace targeted for $E_{i}$ is fine.

- If $m \neq n$ but $i=j$, then $x$ is targeted for $F_{i}$; we cannot appoint a trace for $E_{i}$. But we can appoint a trace $y_{0}$, targeted for $H_{n-1}$ (which is different from $\left.H_{m-1}\right)$ and appoint a trace $y_{1}$ for $y_{0}$, targeted for $E_{1-i}$.

- If $m \neq n$ and $i \neq j$ then a trace may be targeted for $E_{1-j}=E_{i}$, about which $\rho$ does not care.

If $\rho$ is an $A$-gate then $\rho$ 's only wish is that two balls targeted for $H_{n}$ s will not cross at the same time. Thus when a ball waiting at $\rho$ 's corral rolls out to the gate, it is assigned a trace for $E_{i}$.

To achieve the technical condition which prohibits more than one ball targeted for some $H_{n}$ to lie in an entourage segment which is waiting at the permitting bin, we stipulate that the lowest gate is always an $A$-gate.

We remark that followers which are targeted for $G_{n}, n \notin H$, do not need any traces, and so, when released from their hole $\eta$, they fall immediately to the permitting bin, where they do not receive a trace for $B$. These balls are considered 
as all other balls as far as permission from $U$ is concerned; when permitted (parallel to case $(3)$ (a) above) they are enumerated into their target set and the agent $\eta$ declares victory.

We also remark that balls targeted for $H_{0}$ do not need traces; nevertheless, whether followers or traces, they need to participate in the machine. For example, when a follower for $H_{0}$ is realized, it is only dropped to the highest gate below its hole; only when its guess $\xi$ is deemed correct is it allowed to roll down to the bin, where it needs a trace for $B$. When permitted it is enumerated into $C_{0}$, gets a new trace for $B$, and is placed in the lowest pregate, awaiting prompt permission.

The positive ordering requirement $G_{j} \leqslant{ }_{\alpha} G_{i} \oplus L$ (if $j \preccurlyeq i$ ) is met by applying the classical strategy. If a follower $x$ for a $D_{j, \Psi}$ requirement is enumerated into $G_{j}$, then it is also enumerated into $L$. If a follower $x$ for an $N_{i, j, \Psi}$ requirement is enumerated into $G_{j}$ then it is also enumerated into every $G_{k}$ for $k$ such that $j \preccurlyeq k$. As is in the classical construction, this does not disturb the machine part because the $h_{n} \mathrm{~s}$ are all $\preccurlyeq$-minimal.

Whenever any trace $y$ (which is not a follower) is enumerated into a set $H_{n}$ then it is also enumerated into $L$. Again as is in the classical case, this does not disturb the realization of $y$ 's follower even if the associated requirement is an $N$-requirement; this is because $y$ is appointed after $x$ is realized (the first trace appointed for $x$ is targeted for $E_{i}$ and not for any $H_{n}$ ) and so is too large to disturb that realization.

Priority. A follower $x$ is permitted at $s$ if some $y \leqslant x$ enters $U$ at $s$; it is promptly permitted at $s$ if some $y \leqslant x$ enters $U$ by stage $p_{U}(s)$, where $p_{U}$ is a recursive function witnessing the fact that $U$ permits promptly, with respect to the various r.e. sets we use in the verifications. See the remarks after the proof of lemma E.2. 
Just as in the classical construction, for any hole $\eta$, we can calculate a finite number which is the number of permissions (either regular or prompt) a follower issued by $\eta$ needs before it is enumerated into its target. We can therefore assign (shifting) priorities as is done in [NSS98]. The priority of $x$ is determined first by the lexicographic priority of $\eta$, then by the lexicographic priority of $\xi(x)$, next by the number of permissions it has already received (the more the merrier), and finally by its size (which is the same as by the date of birth, as new followers are chosen large). As in the classical construction, when a follower receives permission it might need to cancel other followers for the same hole, which were previously stronger.

As usual, if some node $\eta^{\prime}$ which lies to the left of $\eta$ (i.e. is smaller with regard to the lexicographic ordering on the tree induced by the natural ordering on $\alpha$ ) is accessible, or balls issued by $\eta^{\prime}$ receive attention, then $\eta$ is initialized and all of its followers are cancelled (removed from the machine and never considered again). Also as usual, if a ball $x$ issued by $\eta$ receives attention then all balls issued by $\eta$ which are weaker than $x$ (according to the priority system just described) are cancelled.

However, we also cancel a ball $x$ which belongs to node $\eta$ at a stage $s$ at which the following occurs: at $s$, there is a node $\eta^{\prime} \subsetneq \eta$ which is accessible, $r\left(\eta^{\prime}\right)[s]=0$ but $\xi(x)\left(\operatorname{dom} \eta^{\prime}\right)>0$. It makes sense to do so, because at $s$ we discover that $x$ 's guess about the final value of $r\left(\eta^{\prime}\right)$ (this is the liminf of $r\left(\eta^{\prime}\right)[s]$ ) is incorrect: either $r\left(\eta^{\prime}\right)=0$ is the correct guess; but even if not, then $r\left(\eta^{\prime}\right) \geqslant s$ and clearly $\xi(x)\left(\operatorname{dom} \eta^{\prime}\right)<s$. When cancelling a follower $x$ for this reason, we do not initialize $\eta$ or any weaker agents, nor do we cancel other followers for $\eta$ which may be weaker than $x$ but whose guess is not yet found to be incorrect. 
The following fact, which is true in the classical construction, has even greater importance here.

Lemma 3.3. Let $y, z$ be two balls which are on the machine at some stage s and which belong to different entourages. Then $y$ is stronger than $z$ (at s) iff $y<z$.

Proof. Say that $y<z$. Since new balls are chosen large, $y$ is appointed before

$z$. When $z$ is appointed, $y$ is stronger than $z$ (or $y$ gets cancelled immediately). $z$ in fact remains weaker than $y$ as long as $y$ is left on the machine, since $z$ 's strengthening beyond $y$ will immediately lead to $y$ 's cancellation. Note also that as long as $z$ is on the machine, $y$ cannot receive attention.

This shows that at any given stage, the priority ordering on balls is a wellordering. During the construction we will need to pick, among a perhaps infinite set of balls which require attention, the strongest such ball, and this gives us the justification to do so.

\section{SW Ingredients}

As in the classical construction, the part of the construction which is devoted to building the SW set parameters (namely those agents which work for $K, M$ and $T$ requirements) has very little interaction with the machine. We can therefore make the same definitions for these elements of the construction, remembering that we are working above $B$.

$T$ Requirements. Suppose that pregate $\rho$ is assigned to $T_{n} . \rho$ defines a functional $\Gamma_{\rho}$, with the intention of having $\Gamma_{\rho}\left(G_{n} \oplus P\right)=Q . \rho$ may only add an axiom to $\Gamma_{\rho}$ when it is accessible. 
All uses of $\Gamma$ functionals are successor ordinals; $\gamma_{\rho}(x)=\gamma_{\rho}\left(G_{n} \oplus P, x\right)$ denotes the length of the use of the computation $\Gamma_{\rho}\left(G_{n} \oplus P, x\right)$ minus one, so enumerating it into $G_{n}$ or $P$ destroys the computation.

Chits. Suppose that agent $\eta$ works for $M=M_{\Theta, \Phi, W} \cdot \eta$ defines weak functionals $\Delta_{\eta, j}$ for every $j \leqslant n(\eta)$, with intended oracle $B \oplus W$; the intention is that if the hypothesis of $M$ holds then for some $j \leqslant n(\eta)$ we'll have $\Delta_{\eta, j}(B \oplus W)={ }^{*} G_{j}$.

$\eta$ is allowed to extend the definition of $\Delta_{\eta, j}(B \oplus W)$ (i.e. to enumerate a new axiom into $\Delta_{\eta, j}$ ) at stages which are $\eta$-expansionary. As is implies by the intention, $\eta$ always defines the value of $\Delta_{\eta, j}(B \oplus W, x)[s]$ to be $G_{j}(x)[s]$.

$\eta$ makes $\Delta_{\eta, j}$ monotone, so that at any stage we'll have dom $\Delta_{\eta, j}(B \oplus W)$ an ordinal. If $\eta$ wishes to extend $\Delta_{\eta, j}$ at $s$ then it defines one new axiom on the input $x=\operatorname{dom} \Delta_{\eta, j}(B \oplus W)[s]$ (we'll ensure that the use is larger than the uses $\delta_{\eta, j}(B \oplus W, y)[s]$ for all $y<x$ so that indeed monotonicity is maintained.)

To each computation $(\zeta \oplus \sigma ; x, l) \in \Delta_{\eta, j}$ is associated a chit $(y, \pi)$ (we sometimes also refer to $y$ as the chit). When $\eta$ wishes to define $\Delta_{\eta, 0}(x)$ at stage $s$, it picks a new suitable chit. A chit $(y, \pi)$ is suitable to be picked for a new computation $\Delta_{\eta, 0}(B \oplus W, x)[s]$ if:

1. $y \in \alpha^{[\eta]}$.

2. $y<\ell(M)[s]$.

3. $\phi(B \oplus W \oplus P, y)[s]=\zeta \oplus \sigma \oplus \pi$.

4. $y>\operatorname{init}(\eta)[s]$ and $y>t$ for any $t<s$ at which $\eta$ defined any $\Delta_{\eta, 0}$ computation. 
If $s$ is $\eta$-expansionary and there is a suitable chit $y$, then it defines $\Delta_{\eta, 0}(B \oplus W, x)$ with use $\zeta \oplus \sigma$.

Now let $j>0$. Suppose that at $s, \eta$ wishes to define $\Delta_{\eta, j}(B \oplus W, x)$. To do so, it needs to find a chit $(y, \pi)$ which is suitable to be picked for this computation (we also say that the chit is $j$-suitable). The suitability conditions are:

1. $(y, \pi)$ is a chit for a computation $\Delta_{\eta, j-1}\left(B \oplus W, x^{\prime}\right)[s]$ (whose use is $\left.\zeta \oplus \sigma\right)$.

2. Further, that chit is still active, which means that $\pi \subset P[s]$ (an inactive chit is also called cancelled).

3. The computation $\Delta_{\eta, j-1}\left(B \oplus W, x^{\prime}\right)[s]$ is failed, which means that its value disagrees with $G_{j-1}\left(x^{\prime}\right)$ (necessarily $x^{\prime}$ entered $G_{j-1}$ at some stage after the computation was defined).

4. The size condition: $y>t$ for any $t<s$ at which $\eta$ defined any $\Delta_{\eta, j}$ computation.

If such a chit is found then $\eta$ defines $\Delta_{\eta, j}(B \oplus W, x)[s]$ with the same use $\zeta \oplus \sigma$ as the accompanying computation $\Delta_{\eta, j-1}\left(B \oplus W, x^{\prime}\right)[s]$.

If a computation $\Delta_{\eta, j}(B \oplus W, x)$ which is defined at $s$ becomes incorrect at a later stage $t$ (that is some number smaller than the use enters $B$ or $W$ ) then the accompanying chit $(y, \pi)$ is cancelled and never considered again. Note again that if $(y, \pi)$ is a chit for $\Delta_{\eta, j}\left(B \oplus W, x_{j}\right)$ for $j \leqslant i$ (where $\left.i \leqslant n(\eta)\right)$ then the uses of all of these computations are the same and so all such computations disappear together). However, (2) shows that the chit may be cancelled even if the computations still hold.

At stage $s, \eta$ may wish to use a chit $(y, \pi)$ for purposes of victory. Suppose that $\rho \subset \eta$ is a pregate which works for $T_{n}(n(\rho)=n)$. A chit $(y, \pi)$ is cleared by $\rho$ 
at stage $s$ if $\neg\left(\Gamma_{\rho}\left(B \oplus G_{n} \oplus P, y\right) \downarrow=0[s]\right)$, or if $\Gamma_{\rho}\left(B \oplus G_{n} \oplus P, y\right) \downarrow=0[s]$ with use $\gamma_{\rho}(y)[s]>\operatorname{dom} \pi$. The chit $y$ is victorious if it is $M$-confirmed at $s$, still active, is greater than $\operatorname{Rest}(\eta)[s]$, and is cleared by all pregates $\rho$ below $\eta$.

Pointers. An agent $\eta$ working for a requirement $K_{\Xi, K}$ keeps a pointer $i(\eta)[s]$, which is the next element of $K$ of which it needs to take care. Unless initialized, at a limit stage $s$ we have $i(\eta)[s]=\min \left(K \backslash \sup \{i(\eta)[t]\}_{t<s}\right)$. When $\eta$ is initialized we set $i(\eta)=\min K$. If $\eta$ acts at $s$ then we update the pointer: $i(\eta)[s+1]=$ $\min (K \backslash i(\eta)[s]+1)$. If $i(\eta)[s]=\max K$ and $\eta$ acts at $s$ then $\eta$ declares victory.

\subsubsection{Construction}

Each stage of the construction is divided into three phases. At the first phase, agents working for lowness requirements act; as in the construction of chapter 2 , this is done even before $\delta[s]$ is calculated because of the incompatibility between lowness and the tree construction. At the second phase, the path of accessible nodes is computed and then finitary-type action takes place: balls moving on the machine, numbers enumerated into sets, new followers being appointed, agents declaring victory. At the third phase, agents along $\delta[s]$ enumerate new axioms into the functionals they are building (but further initializations may occur).

We emphasize that at any stage $s$ (and any phase of that stage), if $\eta$ is an agent which declared victory at some stage $t<s$ and $\eta$ was not initialized between $t$ and $s$, then $\eta$ does not require attention at $s$ and does not make any action.

As in chapter 2 we let $\operatorname{init}(\eta)[s]$ be the supremum of stages $t<s$ at which $\eta$ is initialized. 


\section{First Phase}

An agent $\eta$ is allowed to act at the first phase of stage $s$ if there was a stage $t<s$ at which $\eta$ was accessible, such that between $t$ and $s$, neither was $\eta$ initialized, nor did it act. An agent $\eta$ on the tree, working for $K_{\Xi, K}$, wishes to act at the first phase of $s$ if

$$
i(\eta) \in \Xi(R \oplus P \oplus Q \oplus L)[s]
$$

We let every agent $\eta$ which is allowed to act and wishes to act initialize all agents on the tree which are weaker than $\eta$ (again we note that the priority (lexicographic) ordering on agents is linear but not well-founded, so there may not be a strongest node which acts; nevertheless all such $\eta$ must either act or be initialized, so we let them all act, and of course if there is no strongest one they all get intialized.) We also update $i(\eta)$ as described in section 3.1.1. Also, $\eta$ may declare victory.

\section{Second Phase}

First, we calculate the path of accessible nodes $\delta[s]$. Agents which are weaker than $\delta[s]$ are initialized. We also calculate the restraint which accessible agents impose, and cancel balls whose guess about the restraint imposed is found to be incorrect.

We say that a follower $x$ on the machine (which is not neccessarily issued by an accessible agent; it could be an agent to the left of $\delta[s])$ requires attention at $s$ if one of the following holds.

1. The end of $x$ 's entourage is waiting at a gate $\rho$, and $\rho, \xi(x) \uparrow \operatorname{dom} \rho$ are accessible.

2. $x$ is waiting at its appointing hole $\eta$ and is realized; $\eta$ is accessible [Note that we do not require that $\xi(x)$ be accessible]. 
3. The end of $x$ 's entourage is waiting in a cage associated with a pregate $\rho$, and $\rho, \xi(x)\lceil\operatorname{dom} \rho$ are accessible.

4. The end of $x$ 's entourage is waiting in the permitting bin and $x$ is permitted by $U$ at $s$.

If a follower $x$ which was issued by agent $\eta$ requires attention then we also say that $\eta$ requires attention. In addition, an accessible hole $\eta$ which works for an $N$ or $D$ requirement requires attention if:

5. No follower for $\eta$ is waiting at the hole $\eta$, and no other follower for $\eta$ requires attention.

An accessible agent $\eta$ which works for $M=M_{\Theta, \Phi, W}$ requires attention if:

6. There is some $\sigma \subset \Theta(R)[s]$ and some $x<\operatorname{dom} \sigma$ s.t. $\sigma(x)=0 \quad \& \quad x \in W[s]$, or if

7. $r(\eta)[s]=0$ and there is a victorious chit $y$ for $\eta$ which is promptly permitted by $U$.

We already noticed that the balls on the machine at this phase are well-ordered, and so there is a strongest ball which requires attention. Also, $\delta[s]$ is well-ordered, and only accessible nodes require attention independently (i.e. not via any follower). Thus there is, overall, a strongest agent $\eta$ which requires attention. If some follower for $\eta$ requires attention then we let $x$ be the strongest such follower. We initialize agents weaker than $\eta$ and cancel balls weaker than $x$ (note that $x$ may have just received new permission so it may cancel balls which were up to now stronger).

In any case, we let $\eta$ act according to the relevant case. 
1-4. $\eta$ acts according to the instructions above (section 3.1.1). Recall that in case 2 , if $x$ is not targeted for any $H_{n}$ then we simply put it in the permitting bin.

5. $\eta$ appoints a new, large follower $x$, and if necessary, appoints a trace.

6. $\eta$ initializes weaker requirements and declares "easy" victory.

7. $y$ is enumerated into $Q$, and for all pregates $\rho \subset \eta$, if $\Gamma_{\rho}\left(G_{n(\rho)} \oplus P, y\right) \downarrow=0[s]$, then $\rho$ puts $\gamma_{\rho}(y)[s]$ into $P . \eta$ declares victory.

\section{Third Phase}

At the third phase, every accessible agent (which was not just initialized) working for $M$ or $T$ may extend the functionals it defines.

An accessible pregate $\rho$ extends $\Gamma_{\rho}$ by defining it on the least $x$ such that $\Gamma_{\rho}\left(G_{n_{\rho}} \oplus P, x\right) \uparrow[s]$, with new large use and value $Q(x)[s]$.

An accessible agent $\eta$ which works for $M_{\Theta, \Phi, W}$ is allowed to define new $\Delta_{\eta, j}$ computations only if $s$ is $\eta$-expansionary. The definitions are as described above. Further, if $\eta$ defines $\Delta_{\eta, j}(x)$ and $\eta^{\prime} \supset \eta$ is an agent which has $x$ as a ball in one of its entourages, then $\eta^{\prime}$ initializes all agents weaker than itself.

That's the end of the construction.

\subsection{Verifications}

\section{True Path}

The true path is obtained effortlessly as in section 2.3.3, because of the properties of the approximations $p[s]$ and $f[s]$. However, unlike that section, we do need an inductive definition of $\delta$. 
Lemma 3.4. Let $\eta$ be an agent on the tree. Suppose after some stage $s^{*}$, no agent to the left of $\eta$ is ever accessible. Then

$$
\left\{t \geqslant s^{*}: \eta \subset \delta[t]\right\}
$$

is closed.

Proof. Suppose not. Let $t>s^{*}$ be a stage which is a limit of stages at which $\eta$ is accessible, and suppose that $\eta$ is not accessible at $t$. It is impossible that $\delta[t]$ is properly contained in $\eta$, because the construction of $\delta[t]$ is halted only when we get to a node which is not an agent; $\eta$ (and every $\eta^{\prime} \subset \eta$ ) is an agent. It follows that $\eta$ lies to the left of $\delta[t]$. Let $\beta$ be the least such that $\eta(\beta) \neq \delta(\beta)[t]$; so $\eta(\beta)<\delta(\beta)[t]$. Let

$$
\epsilon=\epsilon(\eta \uparrow \beta+1)=\epsilon(\delta \uparrow \beta+1[t])
$$

We, of course, have two cases.

1. $\beta$ is odd. In this case, we know that the set of stages $s<t$ at which $p(\epsilon)[s]=\eta(\beta)$ is unbounded in $t$; this happens at each stage at which $\eta$ is accessible, by the definition of $\delta[s]$. However, $p(\epsilon)[t]=\delta(\beta)[t]>\eta(\beta)$. This can only happen if $\eta(\beta)=0$ but the set of $s$ such that $p(\epsilon)[s]=0$ is closed; contradiction.

2. $\beta$ is even. Let $n=n(\eta \uparrow \beta) . \eta \uparrow \beta \subset \delta[t]$ implies that $\eta$ 's and $\delta[t]$ 's opinion on $f \uparrow n$ are the same: for all $m<n, f(m)[t]=f(m)[s]$ where $s$ is any stage at which $\eta$ is accessible. However, $\eta(\beta)<\delta(\beta)[t]$ again implies that $\eta(\beta)=0$, that is, $f(n)[s]=\epsilon$ whenever $\eta$ is accessible but $f(n)[t]>\epsilon$. But again, if for unboundedly in $t$ many $s$ we have $f(n)[t]=\epsilon$ then we must have $f(n)[t] \leqslant \epsilon ;$ contradiction. 
Lemma 3.5. There is a true path $\delta: \lambda \rightarrow \alpha$ such that for all $\beta<\lambda$,

1. $\delta \uparrow \beta$ is $\alpha$-finite and is in fact an agent on the tree.

2. $\delta \uparrow \beta$ is accessible unboundedly often.

3. There is some stage $s<\alpha$ after which no agent $\eta$ which lies to the left of $\delta \uparrow \beta$ is ever accessible.

Proof. By induction on $\beta<\lambda$ we define $\delta$ up to $\beta$ and show that (1-3) hold for the nodes defined.

We first notice that if for some $\gamma, \delta \uparrow \gamma$ is defined and satisfies (1-3), then by lemma 3.4, the set of stages after $s$ (which is given by (3)) at which $\delta \uparrow \gamma$ is accessible is a (recursive) club.

Suppose that $\delta \uparrow \beta$ is defined and that (1-3) hold for $\delta \uparrow \beta$; we define $\delta(\beta)$. Let $\epsilon=\epsilon\left((\delta \uparrow \beta)^{\frown} 0\right)$. If $\beta$ is even, then if $\epsilon \in$ range $f$ then $\delta(\beta)=0$, otherwise $\delta(\beta)=1$. If $\beta$ is off and $\epsilon \notin \operatorname{dom} p$ then $\delta(\beta)=0$, otherwise $\delta(\beta)=p(\epsilon)$.

We now show that (1-3) hold for $\delta \uparrow(\beta+1)$. (1) is immediate. For $(2)$, let $C$ be a recursive club of stages at which $\delta \uparrow \beta$ is accessible. If $\beta$ is even then let $E$ be a recursive club of stages at which $\epsilon \in$ range $f[s]$ is correct; if $\beta$ is odd let $E$ be a recursive club of stages at which $p(\epsilon)[s]=p(\epsilon)$ (or $p(\epsilon)[s]=0$ if $\epsilon \notin \operatorname{dom} p)$. Then on $C \cap E, \delta \uparrow(\beta+1)$ is accessible.

For (3), suppose that after $s_{0}$, no agent to the left of $\delta \uparrow \beta$ is ever accessible. If $\delta(\beta)=0$ then clearly after $s_{0}$ no node to the left of $\delta \uparrow(\beta+1)$ is ever accessible. Otherwise, if $\beta$ is even, choose $n$ minimal such that $f(n) \geqslant \epsilon$; then $f(n)>\epsilon$ and we can find some $s_{1}>s_{0}$ after which we always have $f(n)[s]>\epsilon$. Then if $s>s_{1}$ and $\delta \uparrow \beta$ is accessible at $s$, then at $s, \epsilon$ is not guessed to be in the ranges of $f$ and so $(\delta \uparrow \beta)^{\frown} 0$ is not accessible at $s$. As $s_{1}>s_{0}$ it follows that no node to the left of 
$\delta \uparrow(\beta+1)$ is ever accessible after $s_{1}$. If $\beta$ is odd, then $\epsilon \in \operatorname{dom} p$ and we can find some $s_{1}>s_{0}$ after which we always have $p(\epsilon)[s]=p(\epsilon)$; after $s_{1}$, no node to the left of $\delta \uparrow(\beta+1)$ is ever accessible.

Now suppose that $\beta$ is a limit ordinal and that $\delta$ is defined up to $\beta$; and that (1-3) hold for $\delta \uparrow \gamma$ for all $\gamma<\beta$. We need to verify that (1-3) hold for $\delta \uparrow \beta$.

For (1), we show that the function $\gamma \rightarrow \delta \uparrow \gamma$, defined on $\beta$, is recursive, hence $\alpha$-finite. We can define this function by induction, using the following sets and functions:

- $\beta \backslash \operatorname{dom} p$

- $p \uparrow(\beta \cap \operatorname{dom} p)$;

- $\beta \cap$ range $f$.

The first is $\Pi_{2}\left(J_{\alpha}\right)$ and so $\alpha$-finite (as $\beta<\lambda$ ); the second is now $\Delta_{2}\left(J_{\alpha}\right)$ and so $\alpha$ finite (using $\Sigma_{2}$-admissibility); the third is finite and so $\alpha$-finite. Now the induction is straightforward; given $\delta \uparrow \gamma, \epsilon\left((\delta \uparrow \gamma)^{\curlyvee} 0\right)$ is computed and $\delta(\gamma)$ is computed as it was defined above we note that always $\epsilon((\delta \uparrow \gamma) \frown 0)<\beta$.

For $\gamma<\beta$ let $s_{\gamma}$ be the least stage witnessing (3) for $\delta \uparrow \gamma$. As $\gamma \rightarrow \delta \uparrow \gamma$ is recursive, $\gamma \rightarrow s s_{\gamma}$ is $\Pi_{1}\left(J_{\alpha}\right)$ and so $\alpha$-finite. For $\gamma<\beta$ let

$$
E_{\gamma}=\left\{s>s_{\gamma}: \delta \uparrow \gamma \subset \delta[s]\right\}
$$

$E_{\gamma}$ is a club, and these clubs are uniformly recursive. It follows that $E=\cap_{\gamma<\beta} E_{\gamma}$ is a club of stages at which $\delta\left\lceil\beta\right.$ is accessible, so (2) holds. Also, $s_{\beta}=\sup _{\gamma<\beta} s_{\gamma}$ witnesses that (3) holds for $\delta \uparrow \beta$.

Lemma 3.6. For every requirement $R$ there is some $\beta<\lambda$ such that $\delta \uparrow \beta$ is assigned to work for $R$. 
Proof. This amounts to showing that for every kind of requirement $X$, the ordertype of the set of $\beta<\lambda$ such that $\delta \uparrow \beta$ is assigned to a requirement of kind $X$, is $\lambda$. This is why we required $f(n+1)>f(n) \cdot 2$. For kind $X$ and every $n$, there is a block of nodes of $\delta$ which has length at least $f(n)$ and is devoted to $X$; so there are at least $f(n)$ many nodes on the true path working for $X$ requirements. As $f$ is cofinal in $\lambda$ we're done.

Define $\chi: \lambda \rightarrow \alpha$ by letting $\chi(\beta)=\liminf _{s} r(\delta \uparrow \beta)[s]$. In fact we know that either there are unboundedly many $\delta \uparrow \beta$-expansionary stages, in which case $r(\delta \uparrow \beta)[s]=0$ on a club of stages and $\chi(\beta)=0$, or from some stage onward, $r(\delta \uparrow \beta)[s]$ is constant (is the supremum of all $\delta \uparrow \beta$-expansionary stages) and equals $\chi(\beta)$.

Lemma 3.7. For all $\beta<\lambda$ the following holds.

1. $\chi \uparrow \beta$ is $\alpha$-finite.

2. $\delta \uparrow \beta, \chi \uparrow \beta$ are accessible on club many stages.

3. There is some stage $t$ after which for all $\gamma<\beta$, whenever $\delta \uparrow \gamma$ is accessible, we have $r(\delta \uparrow \gamma)[s] \geqslant \chi(\gamma)$.

Note that the third condition is stronger than saying that after some stage, whenever $\delta \uparrow \gamma$ is accessible, $\chi[s]$ is not lexicographically to the left of $\chi \uparrow \gamma$, but is immediately implied by the statement that $\chi " \beta$ is bounded below $\alpha$ (any bound witnesses (3)).

Proof. Fix $\beta<\lambda$. Let $K$ be the set of $\gamma<\beta$ such that there are unboundedly many $\eta\left\lceil\gamma\right.$-expansionary stages. $K$ is a $\Pi_{2}\left(J_{\alpha}\right)$-definable subset of $\beta$ which in turn is smaller than $\lambda=\varrho_{\alpha}^{2}$; it follows that $K$ is $\alpha$-finite. 
Now $\chi \uparrow \beta$ is defined as follows:

- If $\gamma \in K$ then $\chi(\gamma)=0$.

- If $\gamma \notin K$ then $\chi(\gamma)=t$ where $t$ is $\delta \uparrow \gamma$-expansionary, or a limit of $\delta \uparrow \gamma$ expansionary stages, but there is no $s>t$ which is $\delta \uparrow \gamma$-expansionary.

As $\gamma \rightarrow \delta \uparrow \gamma$ is recursive, we see that $\chi \uparrow \beta$ is $\Pi_{1}\left(J_{\alpha}\right)$ and so $\alpha$-finite.

Again let $s_{\beta}$ be the least stage after which no node to the left of $\delta \uparrow \beta$ is ever accessible, and let $E_{\beta}$ be the club of stages after $s_{\beta}$ at which $\delta \uparrow \beta$ is accessible. Let $t_{\beta}=\sup \chi " \beta$. For $\gamma \in K$, let $D_{\gamma}$ be the set of stages after $s_{\beta}$ at which $r(\delta \uparrow \gamma)[s]=0$ (i.e. the $\delta\left\lceil\gamma\right.$-expansionary stages or limits of those). $D_{\gamma}$ is a club and the $D_{\gamma}$ s are uniformly recursive. It follows that

$$
E=\left[t_{\beta}, \alpha\right) \cap E_{\beta} \cap\left(\bigcap_{\gamma \in K} D_{\gamma}\right)
$$

is a club of stages at which $\delta \uparrow \beta, \chi \uparrow \beta$ are accessible. Also, $t_{\beta}$ witnesses (3) for $\beta$.

\section{Fairness}

Definition. An agent $\eta \subset \delta$ on the tree is treated fairly if $\operatorname{init}(\eta)[\alpha]<\alpha$. If $\beta<\lambda$ and $\delta \uparrow \beta$ is treated fairly then we let $r_{\beta}^{*}$ be the maximum of $\operatorname{init}(\delta \uparrow \beta)[\alpha]$ and the least stage $t$ witnessing (3) of lemma 3.7

Suppose that $\eta=\delta \uparrow \beta$ is treated fairly, let $r^{*}=r_{\beta}^{*}$, and just for this subsection let $\chi=\chi \uparrow \beta$. We examine how $\eta$ affects weaker agents. The only agents which initialize weaker agents are agents working for $M, K, D$ and $N$ requirements.

If $\eta$ works for an $M$ requirement then $M$ initializes weaker agents at most once after $r^{*}$. 
Lemma 3.8. Suppose that $\eta$ works for $K_{\Xi, K}$. Then after some stage, $\eta$ does not initialize weaker agents, and the requirement is met.

Proof. After $\operatorname{init}(\eta)[\alpha], i(\eta)[s]$ is non-decreasing. Let

$$
K^{\prime}=\{i(\eta)[s]: s>\operatorname{init}(\eta)[\alpha]\}
$$

$K^{\prime}$ is $\alpha$-finite as it is an initial segment of $K$. The function which takes $x \in K^{\prime}$ to the least $s>r^{*}$ at which $i(\eta)[s]=x$ is recursive and so bounded by some $s^{*}$. After $s^{*}, \eta$ does not initialize weaker agents.

If $\eta$ acts at $s>\operatorname{init}(\eta)[\alpha]$ then $i(\eta)[s] \in \Xi(R \oplus P \oplus Q \oplus L)$ is permanently correct. Thus if $\eta$ declares victory after $\operatorname{init}(\eta)[\alpha]$ then the requirement is positively met.

If not, let $i$ be the final value of $i(\eta)[s]$, stabilizing after $s^{*}$. For no $s>s^{*}$ do we have $i \in \Xi(R \oplus P \oplus Q \oplus L)[s]$ and so the requirement is met vacuously.

Suppose then that $\eta$ works for a $D$ or an $N$ requirement.

Observation 3.9. A non-deficiency stage for the construction is a stage $s$ such that no ball on the machine at the end of $s$ will ever receive attention again. There are unboundedly many such stages. For if $t$ is any stage, we can let $x$ be the smallest follower ever to receive attention after $t$ and let $s$ be the last stage at which $x$ receives attention. Now after $x$ receives attention at $s$, all balls left on the machine are at least as strong as $x$, hence not larger; they will never receive attention again. It may be the case that some of these balls will later be cancelled. If $s$ is a non-deficiency stage for the construction, then all sets constructed are correct up to $s$ at $s$, and all computations which are left at the end of $s$ will never be destroyed; this is because these computations have use at most $s$, and the only balls to receive attention after $s$ are those appointed after $s$, which are larger than 
s. Note that this applies only to sets constructed by the machine, namely, $G_{n}, C_{i}$, $E_{i}$ and $L ; P$ and $Q$ are excluded from this discussion.

Lemma 3.10. Eventually, $\eta$ stops appointing new followers.

It follows that there is a stage after which no followers for $\eta$ receive attention.

Proof. Assume the contrary; we compute $U$. We know that no follower is enumerated after $r^{*}$. Of course it cannot be the case that some follower $x$ is appointed after $r^{*}$, is never cancelled, and is never realized at a stage at which $\eta$ is accessible. Also, for every follower $x$ appointed after $r^{*}$, for all $\beta<\operatorname{dom} \eta, \xi(x)(\beta) \geqslant \chi(\beta)$. In fact, if $\xi(x) \neq \chi$ then if not cancelled earlier, $x$ will be cancelled at a stage at which $\eta, \chi$ are accessible. If $\xi(x)=\chi$ then at every stage at which $\eta, \chi$ are accessible and at which $x$ has ball waiting at a gate or in a cage, $x$ will require attention. It follows that no follower can have balls permanently stuck at some gate or in some cage. Thus every follower appointed after $r^{*}$ is either eventually cancelled, or becomes realized and some balls of its entourage get permanently stuck in the permitting bin.

For shorthand, call a follower $x$ for $\eta$ such that $\xi(x)=\chi$ a follower for $\eta, \chi$.

We claim that if $\eta$ appoints unboundedly many followers, then it appoints unboundedly many followers for $\eta, \chi$. Suppose not; suppose that after stage $s_{0}>$ $r^{*}, \eta$ does not appoint any followers $x$ with $\xi(x)=\chi$. Let $s_{1}>s_{0}$ be a nondeficiency stage. Let $s_{2}>s_{1}$ be a stage at which $\eta$ appoints some follower $x$ (so at $s_{2}$, no follower occupies the hole), and let $s_{3} \geqslant s_{2}$ be any stage at which $\eta, \chi$ are accessible. For all $y$ appointed by $\eta$ after $s_{1}$ we have, by assumption, $\xi(y)$ weaker than $\chi$; it follows that at $s_{3}$, all such balls are cancelled. However, all balls appointed before or at $s_{1}$ do not require attention after $s_{1}$. Thus at $s_{3}$, no follower 
for $\eta$ requires attention and the hole at $\eta$ is empty. At $s_{3}$ requires attention and receives it (as $s_{3}>\operatorname{init}(\eta)[\alpha]$ ) and appoints a new follower - contradiction.

Let $m<\omega$ be the largest number such that unboundedly many followers $x$ for $\eta, \chi$ are permitted $m$ many times. Say that all followers for $\eta, \chi$ appointed after $s_{0}>r^{*}$ are permitted at most $m$ times; letting $s_{1}>s_{0}$ be a non-deficiency stage for the construction, we know that all followers $x$ for $\eta, \chi$ which receive attention after $s_{1}$ are permitted at most $m$ times. Also, we note that after $s_{1}$, only followers appointed after $r^{*}$ receive attention, and so if $x$ is a follower which receives attention after $s_{1}$, then for all $\beta<\operatorname{dom} \eta, \xi(x)(\beta) \geqslant \chi(\beta)$.

Claim 3.11. There are unboundedly many followers $x$ for $\eta, \chi$ which at some $s>s_{1}$ are permitted for the $m^{\text {th }}$ time and are never later cancelled by other balls.

Note that followers for $\eta, \chi$ have the correct guess so they can only be cancelled by stronger balls, or by asking for prompt permission and being denied that permission.

Proof. For any non-deficiency stage $s_{2}>s_{1}$, consider the smallest $x$ which is a follower for $\eta, \chi$ and which receives permission for the $m^{\text {th }}$ time at some stage after $s_{2} ;$ suppose this happens at $s_{3}>s_{2}$.

Every ball appointed after $s_{3}$ is always going to be weaker than $x$, as it can never receive more permissions than $x$. Suppose that at the end of $s_{3}, y$ is a ball on the machine. At $s_{3}, y$ is stronger than $x$ and so smaller than $x$. If $y$ does not belong to $\eta$ then it belongs to a stronger node; $y$ will not receive attention later since we are after $\operatorname{init}(\eta)[\alpha]$. If $y$ is a follower for $\eta$ then $\xi(y)$ is at least as strong as $\chi$ (or $y$ is cancelled at $s_{3}$ ). If $\xi(y)$ is stronger than $\chi$ then as mentioned above, $y$ was appointed before $r^{*}$ and so does not receive attention after $s_{1}$. Assume then 
that $y$ is a follower for $\eta$ and that $\xi(y)=\chi$.

$x$ not cancelling $y$ at $s_{3}$ implies that by the beginning of $s_{3}, y$ has already received at least $m$ permissions. By minimality of $x$, the $m^{\text {th }}$ permission for $y$ must have been granted before or at $s_{2}$. As $s_{2}$ is a non-deficiency stage, we know that $y$ does not receive attention after $s_{2}$ and so will never cancel $x$.

We note that all entourages for $\eta$ follow exactly the same path down the tree; this is because we let balls wait at gates even if those gates are occupied by stronger balls. It follows that the status of the entourage (last segment waiting at a gate or in a cage) after receiving $m$ permissions is the same for all followers for $\eta$. We claim that after receiving $m$ permissions, it cannot be the case that the last segment lies in a cage.

Suppose not; then after receiving the $m^{\text {th }}$ permission, followers have balls waiting in the cage behind pregate $\rho$. There are unboundedly many followers $x$ for $\eta, \chi$ with the following property: At some stage $s_{2}>s_{1}, x$ is permitted for the $m^{\text {th }}$ time and at the next stage $s_{3} \geqslant s_{2}$ at which $\rho, \chi \uparrow \operatorname{dom} \rho$ is accessible, $x$ is still on the machine, requires attention and receives it (any follower which is permitted $m$ times and not cancelled by stronger balls would fit this description). Now none of these balls can be permitted once more, so all are cancelled when at $s_{3}$ they ask for prompt permission. However, the set of such $x \mathrm{~s}$ is recursively enumerable; this contradicts the fact that $U$ is promptly simple.

Let $\varphi(x, s)$ be the statement: " $x$ is a follower for $\eta, \chi$ and $s>s_{1} . \eta, \chi$ are accessible at $s$. At the end of $s, x$ is on the machine (uncancelled) and has some balls waiting in the permitting bin. Further, before $s, x$ has already been permitted $m$ times." $\varphi$ is computable.

The analysis above shows that for every follower for $\eta, \chi$ which is permitted for 
the $m^{\text {th }}$ time at some stage after $s_{1}$ and not later cancelled by stronger balls, there will be some $s$ such that $\varphi(x, s)$ holds: at the last stage at which such a follower $x$ receives attention, balls of its entourage are placed in the permitting bin where they are to reside for ever. It follows that there are unboundedly many followers $x$ such that at some $s, \varphi(x, s)$ holds.

We show that if $\varphi(x, s)$ holds then $x$ will in fact never be cancelled after $s$, and so that $U \uparrow x[s]=U \uparrow x$ - this gives us an algorithm to calculate $U$. Take such $x$ and $s$. Of course, we know that $x$ will not be permitted again, and so will not receive attention again and will not be cancelled at a later stage due to lack of prompt permission. Also, as noted earlier, $x$ 's guess $\xi$ is correct and so $x$ will not be cancelled due to finding its guess is incorrect.

Let $t \leqslant s$ be the stage at which $x$ last receives attention (at $t$, balls in $x$ 's entourage are placed in the bin). Balls which at the end of $t$ are weaker than $x$ are cancelled at $t$; as above, new balls appointed after $t$ will always be weaker than $x$ since they cannot be permitted more times than $x$. Consider a ball $y$ which at the end of $t$ is stronger than $x$. If $y$ is not a follower for $\eta$ then $y$ cannot receive any attention after $t$. Suppose that $y$ is a follower for $\eta$. We know that if $y$ receives attention after $t$ then $\xi(y)(\beta) \geqslant \chi(\beta)$ for all $\beta<\operatorname{dom} \eta$. If $\chi \neq \xi(y)$ then $y$ is weaker than $x$ and so is cancelled at $t$. We thus assume that $y$ is a follower for $\eta, \chi$

By $t, y$ has already been permitted $m$ times, and so either has balls in the bin (in which case it will never receive attention after $t$ and will thus never cancel $x$ ) or has balls waiting at a gate $\rho$. The latter is impossible though: if that were the case, then at some stage after $t$, but not later than $s, \rho, \chi \uparrow \rho$ is accessible and $y$ requires attention and cancels $x$. 
Corollary 3.12. $\eta$ satisfies its requirement.

Proof. Suppose that at $s>\operatorname{init}(\eta)[\alpha], \eta$ enumerates a follower $x$ into its target set and declares victory. At $t<s$ the follower is found to be realized and is dropped from the hole; it cancels weaker balls. Smaller (stronger) balls do not receive attention until $s$, since that would cancel $x$. All balls appointed later are larger than $t$, hence larger than the use of the computation which realizes $x$. Also, $x$ 's first trace is targeted for some $E_{i}$ and so does not destroy the realizing computation; all other traces are appointed after $t$ so are too large to destroy that computation. Thus $x$ is still realized at $s$. At $s$, all other balls associated with $\eta$ are cancelled and $\eta$ never acts again. Agents weaker than $\eta$ are initialized at $s$ and later appoint large balls. Agents stronger than $\eta$ never act after init $(\eta)[\alpha]$. It follows that the computation realizing $x$ is preserved for ever and so the requirement is positively met.

Suppose this does not happen. After stage $s_{0}>r^{*}, \eta$ appoints no new followers, and no followers for $\eta$ receive any attention. There is a stage $s_{1}>s_{0}$ after which no balls associated with $\eta$ are ever cancelled (the set of such balls is r.e., hence $\alpha$ finite). Let $s_{2}>s_{1}$ be a stage at which $\eta$ is accessible. At that stage, there must be a follower $x$ for $\eta$ which occupies the hole, unrealized; otherwise, $\eta$ would appoint a follower at $s_{2}$, as no follower requires attention at $s_{2} . x$ is never cancelled and never receives attention. It follows that $x$ is not correctly realized - if it is at some stage, then at the next stage at which $\eta$ is accessible, $x$ requires attention, even if its guess is incorrect (too strong). Thus the requirement is met negatively.

Suppose that $\rho \subsetneq \eta$ works for $M=M_{\Theta, \Phi, W}$; let $j \leqslant n(\rho)$. What follows is identical to the arguments given in section 2.2.4 (observation 2.10 and its sequel). 
Observation 3.13. Suppose that $s>t>\operatorname{init}(\rho)[\alpha]$. Suppose that $\Delta_{\rho, j}(B \oplus W, x) \downarrow$ $[t]$ with use $\zeta \oplus \sigma$, that $\zeta \subset B[s]$ and that $\sigma \subset \Theta(R)[s]$. Then $\rho$ does not redefine $\Delta_{\rho, j}(x)$ at stage $s$. For if it does, then $\Delta_{\rho, j}(B \oplus W, x) \uparrow[s]$ which means that $\sigma \not \subset W[s]$ anymore; necessarily some $y<\operatorname{dom} \sigma$ entered $W$ between $t$ and $s$. But then $\rho$ can get an easy victory at $s$ and defines nothing.

Let $K(\eta)$ be the final set of balls issued by $\eta$ which are never cancelled (it is $\alpha$-finite), and let $r^{* *}>r^{*}$ be a stage after which $\eta$ neither appoints any followers, nor does any follower receive attention or get cancelled. Suppose that $x \in K(\eta)$ and suppose that at some stage $s>r^{* *}, \rho$ defines $\Delta_{\rho, j}(x)$, say with use $\zeta \oplus \sigma$. At some stage $t>\operatorname{init}(\eta)[\alpha], x$ is chosen as a follower for $\eta$. Since large followers are chosen, we must have $t<s$; so $\eta$ initializes weaker agents at $s$. $\eta$ 's action at $s$ ensures that $\sigma \subset \Theta(R)$ and $\zeta \subset B$ from $s$ onwards (this is because $s>r^{* *}$ ). It follows that $\rho$ will not redefine $\Delta_{\rho, j}(x)$ at any other stage after $r^{* *}$.

Let $K^{\prime}(\eta, \rho, j)$ be the set of followers $x \in K(\eta)$ such that $\eta$ initializes for the sake of a $\Delta_{\rho, j}(x)$ computation after stage $r^{* *} . K^{\prime}(\eta, \rho, j)$ is an r.e. subset of $K(\eta)$ hence is $\alpha$-finite. By $\Sigma_{2}$-admissibility, we know that there is a bound to the stages at which $\eta$ initializes weaker agents because $\rho$ defines a $\Delta_{\rho, j}$ computation.

Also, the set of pairs $(\rho, j)$ for which $\eta$ ever initializes is $\alpha$-finite; again by $\Sigma_{2^{-}}$ admissibility, we know that after some stage, $\eta$ stops initializing on behalf of any stronger $\rho$.

We have proved:

Corollary 3.14. If $\eta$ is any node which is treated fairly, then after some stage $\eta$ doesn't initialize weaker nodes.

Now fairness follows easily.

Lemma 3.15. Every node on the true path is treated fairly. 
Proof. By induction on $\beta<\lambda$, we show that $\operatorname{init}(\delta \uparrow \beta)[\alpha]<\alpha$. Suppose up to $\beta$. There are two kinds of nodes which may initialize $\delta \uparrow \beta$.

- Nodes $\delta \uparrow \gamma$ for $\gamma<\beta$. By induction and by corollary 3.14, for each such $\gamma$, there is some stage $s$ after which $\delta \uparrow \gamma$ does not initialize weaker nodes. Let $s_{\gamma}$ be the least such stage; $\gamma \rightarrow s_{\gamma}$ is $\Pi_{1}\left(J_{\alpha}\right)$ hence bounded.

- Nodes $\eta$ which lie to the left of $\delta \uparrow \beta$. We know that after some stage $s^{*}$, no such node is ever accessible. Thus the set of nodes which are to the left of $\delta \uparrow \beta$ and are ever accessible is $\alpha$-finite. After $s^{*}$, each such node may act at most once; the set of nodes which do is r.e. and so $\alpha$-finite. It follows that after some stage, no node which lies to the left of $\delta \uparrow \beta$ initializes other nodes.

Corollary 3.16. Every finitary-type requirement (that is, a requirement of type $N, D, K)$ is met.

\section{Positive Ordering Requirements}

A number $y$ goes into $H_{n}$ iff $y$ enters $C_{1}^{[n]}$; so $H_{n} \leqslant \alpha C_{1}$.

Lemma 3.17. For every $n, H_{n} \leqslant{ }_{\alpha} C_{0}$.

Proof. Let $\rho$ be the $n^{\text {th }}$ pregate on the true path, and let $r^{*}$ be the stage which witnesses that $\rho$ is treated fairly. Let $\chi$ be the correct guess of outcomes up to $\operatorname{dom} \rho$.

Every $y$ which enters $H_{n}$ also earlier enters $C_{0}^{[n]}$. On the other hand, suppose that $y$ enters $C_{0}^{[n]}$ at some stage $s_{0}>r^{*}$ (and is placed in some cage). Let $s_{1}$ be the least stage after $s_{0}$ at which $\rho, \chi$ are accessible. We claim that either $y$ is enumerated into $H_{n}$ at $s_{1}$ (or earlier), or it is never enumerated into $H_{n}$. As we 
can find $s_{0}$ from $y$ using the $C_{0}$ oracle and can find $s_{1}$ from $s_{0}$ effectively, this gives us the required reduction.

Suppose then that $y$ is not enumerated into $H_{n}$ by stage $s_{1}$. If $y$ was issued by a node to the right of $\rho$ then it is cancelled by $s_{1}$. Otherwise, at the beginning of $s_{1}, y$ is waiting in $\rho$ 's cage. If $\xi(y)$ is to the left of $\chi$ then we know that after $s_{1}, y$ will never require attention and so will not end up in $H_{n}$. If $\xi(y)$ lies to the right of $\chi$ then $y$ is cancelled by $s_{1}$. If $\xi(y)$ extends $\chi$ then at $s_{1}, y$ requires attention. If it is not enumerated into $H_{n}$ then it must be cancelled at $s_{1}$ (by a stronger ball, or because of failure to be promptly permitted).

Lemma 3.18. For every $n>0, H_{n} \leqslant{ }_{\alpha} H_{n-1} \oplus E_{n} \bmod 2$.

Proof. This is classical. Whenever $x$ is a ball on the machine targeted for $H_{n}, x$ has a trace $y$ targeted for either $H_{n-1}$ or for $E_{n} \bmod 2 . x$ does not enter $H_{n}$ unless $y$ enters its target set; and there are only finitely many such traces appointed.

Exactly as before, if $j \preccurlyeq i$, then $G_{j} \leqslant \alpha G_{i} \oplus L$. Also, as balls entering any set need permission (either prompt or regular), all sets are recursive in $U$.

\section{Infima}

Let $\eta$ be an agent on the true path which works for an infimum requirement $Z \in$ $\left\{A_{\Phi}, Y_{n, \Phi}\right\}$. In the first case, let $X_{0}=C_{0}, X_{1}=C_{1}$ and $X=H_{<n(\eta)}=\oplus_{k<n(\eta)} H_{k}$. In the second case, let $X_{0}=H_{n-1} \oplus E_{n} \bmod 2, X_{1}=F_{n} \bmod 2$, and $X=H_{n}$.

Suppose that the hypothesis of the requirement holds: $\Phi\left(X_{0}\right)=\Phi\left(X_{1}\right)$ is total; we want to show how to calculate this function from $X$. Let $r^{*}$ witness that $\eta$ is treated fairly and let $\chi$ be the correct guess of outcomes up to dom $\eta+1$. There are unboundedly many $\eta$-expansionary stages, so $\chi(\operatorname{dom} \eta)=r(\eta)=0$ is the correct outcome. 
In the analysis which follows, it is important not only at what stage various events happen but also at what phase. For notational simplicity, we refer to "moments" in time, but write down the stage only. In particular, the moment a certain computation is injured is the exact moment at which the injuring balls enter the relevant set; and the moment at which $\eta$ is expansionary is the beginning of phase two of an $\eta$-expansionary stage, just when $\delta[s]$ is computed and balls are cancelled, but before balls move.

We need to better define some notions and to describe $\eta$ 's unique point of view, which we shall adopt in this subsection. Suppose that $Z=A_{\Phi}$. A ball, targeted for some $H_{n}$, residing at a gate, hole, corral or in the bin is said to be also targeted for $C_{0}$; such a ball crosses $\eta$ when it is released by a gate above $\eta$ and ends up residing below $\eta$. A ball, targeted for some $H_{n}$, which resides in some cage $\rho$, is said to be also targeted for $C_{1}$; such a ball crosses $\eta$ when it is released from the gate and enumerated. In fact, $\eta$ thinks of these as two different balls, the second one issued by $\rho$, rather than by $\eta$.

However, if $Z=Y_{n, \Phi}$, then balls lying in a cage are not considered by $\eta$ to really reside in that cage; in $\eta$ 's eyes, they are still waiting for enumeration at the bottom of the machine, so the notion of a ball lying below $\eta$ depends on $Z$.

Because of that, balls released from a cage above $\eta$ are not considered by $\eta$ to be crossing $\eta$ at that stage. It follows that in either case, in $\eta$ 's eyes, any ball can cross $\eta$ at most once.

Definition. A ball $z$ which is on the machine at stage $s>r^{*}$, is called dormant at that stage if one of the following occurs:

- $z$ belongs to a hole which is stronger than $\eta$. 
- Members of z's entourage are waiting in the bin or in some cage and have a trace for $B$ which is not an element of $B$.

- Members of $z$ 's entourage are waiting at a gate $\rho$ below $\eta$ and $\xi(z)$ is to the left of $\chi\lceil\operatorname{dom} \rho$.

Lemma 3.19. 1. If $z$ is dormant at $s$ then no balls of $z$ 's entourage ever enter any sets; in fact if they ever receive attention after $s$ it is to ask for prompt permission, at which point they are cancelled.

2. If $\eta, \chi$ are accessible at $s$ and $z$ is a ball which lies below $\eta$ at $s$ and never receives attention after $s$, then $z$ is dormant at $s$.

3. The relation " $z$ is dormant at $s$ " is recursive in $B$.

4. If $z$ is dormant at $s, t>s$ and $z$ is not already cancelled at $t$, then $z$ is dormant at $t$.

Proof. (1). Let $z$ be dormant at $s$. If $z$ is issued by a hole which is stronger than $\eta$, then $z$ cannot receive attention after $s$ since $s>r^{*}$. If balls in $z$ 's entourage are waiting in the bin and have a trace for $B$ which does not enter $B$ then $z$ does not receive attention after $s$. If balls in $z$ 's entourage are waiting in some cage and have a trace for $B$ which does not enter $B$, then these balls can never receive permission; they are either cancelled, or never receive attention after $s$. If balls in $z$ 's entourage are waiting at some gate $\rho$ below $\eta$, and $\xi(z)$ is to the left of $\chi \uparrow \operatorname{dom} \rho$, then these balls never receive attention after $s$, because $\xi(x) \uparrow \operatorname{dom} \rho$ is never accessible after $s$.

(2). Suppose that at $s>r^{*}$, a ball $z$ lies below $\eta$ and never receives attention after $s$; suppose also that $\eta, \chi$ are accessible at $s$. There are three possibilities 
for the status of $z$ 's entourage. $z$ may be waiting at a hole, so belongs to a hole stronger than $\eta$. Balls of $z$ 's entourage may be waiting in the bin or in some cage on the machine; the fact that $z$ never receives attention after $s$ implies that the last trace for $B$ will not enter $B$. Finally, balls in $z$ 's entourage may be waiting at some gate $\rho$, necessarily below $\eta$, and $z$ belongs to a hole which extends $\eta$ (if it belongs to a hole to the right of $\eta$ then $z$ is cancelled at $s) . \xi(z)$ is at least as strong as $\chi \uparrow \operatorname{dom} \rho$ since otherwise $z$ gets cancelled at $s$, as its guess is discovered to be incorrect. If $\chi \uparrow \operatorname{dom} \rho \subset \xi(z)$ then $z$ requires attention at $s$. Thus if $z$ never requires attention then it must be the case that $\xi(z)$ lies to the left of $\chi \uparrow \operatorname{dom} \rho$. In all cases we see that $z$ is dormant at $s$.

(3) and (4) are easy.

Let $y<\alpha$. For shorthand, let, for $i<2, u_{i}[s]=\phi\left(X_{i}, y\right)[s]$. Consider a moment $s$ after $r^{*}$ at which the following holds:

1. $\eta, \chi$ are accessible at $s$.

2. $\ell(Z)[s]>y$.

3. For both $i<2, X \uparrow u_{i}[s]$ is correct.

4. Every ball on the machine which lies below $\eta$ at $s$ is dormant at $s$.

Call such a moment $y$-safe. The set of $y$-safe moments is (uniformly in $y$ ) recursive in $X$.

Lemma 3.20. For every $y<\alpha$, there are unboundedly many $y$-safe moments.

Proof. Suppose that after $s_{0}>r^{*}, \ell(Z)>y$ is witnessed by correct $\Phi$ computations. Let $s_{1}>s_{0}$ be a non-deficiency stage for the construction, and suppose that 
$s_{2}$ is the least moment after $s_{1}$ at which $\eta, \chi$ is accessible. We claim that in fact no balls on the machine at $s_{2}$ ever receive attention after $s_{2}$; it will follow that all balls on the machine at $s_{2}$ which lie below $\eta$ at $s_{2}$ are dormant at $s_{2}$.

Suppose that at $s_{2}, z$ is a ball which lies below $\eta$. We claim that $z$ must have been on the machine at the end of $s_{1}$, and so does not receive attention after $s_{1}$. Suppose not. As $\eta$ is accessible at $s_{2}$ and $z$ is not immediately cancelled, we know that $z$ belongs to some node $\eta^{\prime}$ extending $\eta$ (it cannot be appointed by some stronger node after $s_{1}$ since that would initialize $\eta$ ). Also, $\chi \subset \xi(z)$ since otherwise $z$ would be cancelled at $s_{2}$. Further, the follower $x$ to which $z$ belongs must have been appointed after $s_{1}$, since no followers which are on the machine at $s_{1}$ ever receive attention and so do not appoint new traces. But $s_{2}$ is the least stage after $s_{1}$ at which $\eta, \chi$ are accessible, and so $x$ could not have been appointed before $s_{2}$; this is a contradiction.

The following clears a hurdle that does not exist in classical recursion theory.

Lemma 3.21. For every $s>r^{*}$, $s$ is $\eta$-expansionary iff $\eta \in \delta[s]$ and $r(\eta)[s]=0$.

Proof. We need to show that if $s>r^{*}$ is a limit of $\eta$-expansionary stages then it is $\eta$-expansionary. Let $s$ be such a stage, let $s_{0}<s$ be $\eta$-expansionary $\left(s_{0}>r^{*}\right)$ and let $y<\ell(Z)\left[s_{0}\right]$.

As long as we can, we define a double sequence of moments $\left\langle t_{j}, s_{j}\right\rangle_{j<\omega}: t_{j}$ is the least moment after $s_{j}$ (but before $s$ ) at which one of the computations $\Phi\left(X_{0}, y\right)\left[s_{j}\right]$, $\Phi\left(X_{1}, y\right)\left[s_{j}\right]$ is injured. $s_{j+1}$ is the least $\eta$-expansionary moment after $t_{j}$. Note that $s_{j+1}$ is the least moment after $t_{j}$ at which $\eta$ is accessible and $r(\eta)=0$. Also, $s_{j+1}<s$ because $s$ is a limit of $\eta$-expansionary stages. 
We claim that the sequence $\left\langle t_{j}, s_{j}\right\rangle$ is finite. This will imply that after some $t<s$, the computations $\Phi\left(X_{i}, y\right)$ are not injured, which implies that these computations still exist at $s$ (before balls move, but when $\delta[s]$ is calculated). It follows that for every $\eta$-expansionary $s_{0}<s$ we have $\ell(Z)\left[s_{0}\right] \leqslant \ell(Z)[s]$; as $s$ is a limit of expansionary stages, we get that $s$ is $\eta$-expansionary.

Let $x_{j}$ be the ball which injures the relevant computation at $t_{j}$. Let $x$ be

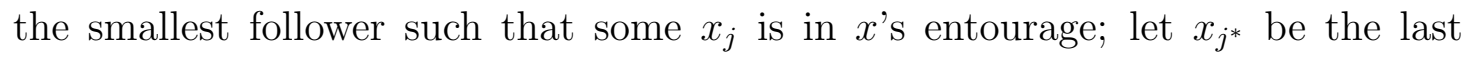
$x_{j}$ which is in $x$ 's entourage. We claim that $x_{j^{*}+1}$ cannot exist. Suppose that it does, and let $x^{\prime}$ be the follower to whose entourage $x_{j^{*}+1}$ belongs. For some $i<2$, $x_{j^{*}+1}<u_{i}\left[s_{j^{*}+1}\right]$ and so $x_{j^{*}+1}$ and $x^{\prime}$ are on the machine at $s_{j^{*}+1}$.

$s_{0}>r^{*}$ so $x^{\prime}$ is issued by a node no stronger than $\eta$. $\eta$ is accessible at $s_{j^{*}+1}$ so $x^{\prime}$ is really issued by a node extending $\eta$; in particular $\operatorname{dom} \eta<\operatorname{dom} \xi\left(x^{\prime}\right)$. Also, $r(\eta)\left[s_{j^{*}+1}\right]=0$ and $x^{\prime}$ is not cancelled at $s_{j^{*}+1}$ so $\xi\left(x^{\prime}\right)(\operatorname{dom} \eta)=0$. This implies that $x^{\prime}$ could not have been appointed between $t_{j^{*}}$ and $s_{j^{*}+1} . x^{\prime}$ is thus on the machine at stage $t_{j^{*}}$, and is not cancelled at $t_{j^{*}}$ by $x$, so $x^{\prime}<x$-contradiction.

Let $s^{*}$ be a $y$-safe stage.

Definition. A moment $t>s^{*}$ is superb, witnessed by $i<2$ and $x<\alpha$, if the following holds at $t$ :

1. At $t$, both computations $\Phi\left(X_{0}, y\right)$ and $\Phi\left(X_{1}, y\right)$ converge.

2. $\eta$ is accessible at $t$ and $r(\eta)[t]=0$.

3. The computation $\Phi\left(X_{i}, y\right)[t]$ is $X$-correct.

4. $x$ is a ball which crosses $\eta$ at $t$, and will injure $\Phi\left(X_{i}, y\right)$.

5. Every ball which lies below $\eta$ at $t$ is dormant. 
Suppose that one of $\Phi\left(X_{0}, y\right)\left[s^{*}\right]$ or $\Phi\left(X_{1}, y\right)\left[s^{*}\right]$ is injured after $s^{*}$; say $\Phi\left(X_{i}, y\right)$ is injured first, by some ball $x$.

Lemma 3.22. $x$ crosses $\eta$ at some moment $t>s^{*}$ which is superb (witnessed by $i$ and $x)$.

Proof. $x$ cannot belong to a node stronger than $\eta$, since $s^{*}>r^{*} . x$ was on the machine at $s^{*}$ since $x<u_{i}\left[s^{*}\right] ; \eta$ is accessible at $s^{*}$ and so $x$ cannot belong to a node to the right of $\eta$; thus $x$ belongs to a node which lies above $\eta$. At $s^{*}, x$ (and its entire entourage) lies above $\eta$ since every ball below $\eta$ at $s^{*}$ is dormant.

It follows that $x$ crosses $\eta$ after $s^{*}$; let $t$ be the moment at which $x$ crosses $\eta$. Of course, neither computation $\Phi\left(X_{j}, y\right)\left[s^{*}\right]$ was injured before $t$ so (1) and (3) hold. Also, $\chi$ is accessible at $s^{*}$ and $x$ is not cancelled at $s^{*}$, so $\xi(x)(\operatorname{dom} \eta)=0$; so $r(\eta)[t]=0$, so (2) holds. It remains to show that (5) holds.

Let $z$ be a ball which lies below $\eta$ at $t$. If $z$ is stronger than $x$ then $z$ does not receive attention between $s^{*}$ and $t$; it follows that $z$ was below $\eta$ at $s^{*}$, so was dormant at $s^{*}$, so it dormant at $t$. Of course $z$ cannot be weaker than $x$. We claim that $z$ cannot belong to $x$ 's entourage.

If $x$ is released from a gate at $t$ then it is clear that all balls of $x$ 's entourage which do not cross $\eta$ together with $x$, lie above $\eta$ at $t$. So assume that $x$ is targeted for $C_{1}$ and is released from a cage at $t$. Let $u$ be the moment at which $x$ was enumerated into $C_{0}$. There are two cases:

- $u<s^{*}$. In this case, between $u$ and $t, x$ 's entourage does not receive attention. At $s^{*}$, no balls in this entourage lie below $\eta$ and so they don't lie below $\eta$ at $t$.

- If $u>s^{*}$ then we know that $x$ must have crossed $\eta$ between $s^{*}$ and $u$ at some 
moment $v$. Now as $\eta$ works in this case for $A_{\Phi}$ we know that $x$ is the smallest ball crossing $\eta$ at $v$ (perhaps with one trace for some $E_{j}$ ). Every $z<x$ which is in $x$ 's entourage is left above $\eta$ at $v$ in some corral, and does not leave the corral until after $t$. Of course every $z>x$ which is in $x$ 's entourage at $t$ crosses $\eta$ together with $x$ at $t$.

Lemma 3.23. If $t$ is superb, witnessed by $i$ and $x$, and $\Phi\left(X_{1-i}, y\right)$ is injured after $t$, then there is another superb moment $v>t$, witnessed by $1-i$ and some $z \leqslant x$, at which the computation $\Phi\left(X_{1-i}, y\right)$ is the same as the computation at $t$.

Proof. Let $u$ be the moment at which $x$ enters $X_{i}$ and let $z$ be the first ball which injures $\Phi\left(X_{1-i}, y\right)[t]$.

Claim 3.24. $z$ enters $X_{1-i}$ after $u$, and immediately after $u, z$ resides above $\eta$.

Proof. We know that $z$ is on the machine at $t$. If $z$ is stronger than $x$ then $z$ does not receive attention between $t$ and $u$; also, it cannot reside below $\eta$ at $t$ (by (5) at $t$ ). $z$ must belong to a node above $\eta$ since $\eta$ is accessible at $t$ (and $t>r^{*}$ ). Thus $z$ resides above $\eta$ at $t$, and so at $u$.

$z$ cannot be weaker than $x$, since then it would be cancelled at $t$. Assume then that $z$ belongs to $x$ 's entourage. We know that every ball in $x$ 's entourage either crosses $\eta$ with $x$ at $t$ or at $t$ lies in a corral above $\eta$. If $z$ does not belong to $x$ 's segment at $t$, then it does not move until $u$, so the claim holds. Suppose then that $z$ crosses $\eta$ together with $x$.

We first claim that we cannot have $Z=Y_{n, \Phi}$. Because in that case, we know that the only way that two balls crossing $\eta$ at the same time, one targeted for $X_{0}$ and the other for $X_{1}$, is if the smaller one is targeted for $X=H_{n}$. We know that $x$ is not targeted for $X$ as $\Phi\left(X_{i}, y\right)[t]$ is $X$-correct. Thus $z<x$; but $X\lceil x$ is correct 
at $t$ and so $z$ cannot enter $X$ - impossible.

If $Z=A_{\Phi}$ then we know that at each time, only one ball targeted for some $H_{n}$ crosses $\eta$ (whether it is released by a gate or a cage). Thus $z=x$. Since $x$ is not targeted for $X=H_{<n(\eta)}$, we know that at $u, x=z$ is retargeted for $C_{1}$ and is placed in a cage which lies above $\eta$.

It follows that $z$ crosses $\eta$ after $u$; let $v$ be the moment at which $z$ crosses $\eta$, we show superbness. (4) holds by definition. For (2), note that $z$ is not cancelled at $t$, at which $\eta$ was accessible and $r(\eta)=0$, so $\xi(z)(\operatorname{dom} \eta)=0$. It follows that at $v, \eta$ must be accessible and $r(\eta)=0$. Lemma 3.21 implies that $v$ is actually $\eta$-expansionary, so (1) holds at $v$.

For (3), we show that $X \uparrow u_{1-i}[t]$ is correct at $t$. This is because $x<u_{i}[t]$ and so $X\left\lceil x\right.$ is correct at $t$, and $x$ eliminates larger balls at $t$ ( $x$ being targeted for $X_{i}$ implies that no immediate trace of $x$ can be targeted for $X$ ). Of course new balls appointed are greater than $u_{1-i}[t]$.

All balls of $z$ 's entourage not crossing $\eta$ together with $z$ at $v$ are, at $v$, lying in corrals above $\eta$. This is the same argument as is given at the end of the proof of lemma 3.22, with $z$ in place of $x, t$ in place of $s^{*}$ and $v$ in place of $t$. Let $b$ be a ball which lies below $\eta$ at $v$. It follows that $b$ is stronger than $z$, and so did not receive attention between $t$ and $v$. It follows that $b$ was below $\eta$ at $t$, and so was dormant then, and so is dormant at $v$. This is (5).

It follows that the value of $\Phi\left(X_{i}, y\right)\left[s^{*}\right]$ is correct, because the sequence of superb stages given by lemmas 3.22 and 3.23 must be finite (as $z \leqslant x$, and each $x$ can occur at most twice); at the last such stage, there is a permanent computation. $\eta$ thus meets its requirement. 


\section{Success of Pregates}

This is exactly as in chapter 2. Let $\eta$ be the pregate on the true path working for $T_{n}$, and let $r^{*}$ witness that $\eta$ is treated fairly.

Claim 3.25. If $\Gamma_{\eta}\left(G_{n} \oplus P, y\right) \downarrow$ then $\Gamma_{\eta}\left(G_{n} \oplus P, y\right)=Q(y)$.

Proof. Let $s>r^{*}$, and suppose that $\Gamma_{\eta}\left(G_{n} \oplus P, y\right) \downarrow[s]$ and that at $s$, agent $\rho$, working for $M_{\Theta, \Phi, W}$ puts $y$ into $Q$. $\rho$ cannot be stronger than $\eta$ since $s>r^{*}$. Also, $\rho$ cannot be to the right of $\eta ; \eta$ defined $\Gamma_{\eta}(y)$ at stage $t<s$ and initialized all nodes to its right at $t$; and new chits $y$ are picked large. Thus $\eta \subset \rho$.

Therefore $\rho$ puts $\gamma_{n}(y)[s]$ into $P$ at $s$, removing the computation $\Gamma_{\rho}\left(G_{n} \oplus P, y\right)$. If the computation is defined later it must be correct.

Claim 3.26. Suppose that $\beta<\alpha$ and that $\Gamma_{\eta}\left(G_{n} \oplus P\right) \uparrow \beta$ stabilizes by some $\alpha$-finite stage. Then $\Gamma_{\eta}\left(G_{n} \oplus P, \beta\right) \downarrow$.

Proof. Suppose that after $t>r^{*}, \Gamma_{\eta}\left(G_{n} \oplus P\right) \uparrow \beta$ is fixed. At every later stage at which $\eta$ is allowed to define $\Gamma_{\rho}$, if $\Gamma_{\rho}\left(G_{n} \oplus P, \beta\right) \uparrow$ then it gets defined, say with use $\tau$. At such a stage enumerate $\tau$ into a functional $\Xi$. Let $\rho$ be the agent which works for $K_{\Xi}$. At some stage $t>s, \Xi$ acts and so preserves $\tau \subset G_{n} \oplus P$.

Lemma 3.27. Every $T$ requirement is met.

Proof. For every $\beta, \Gamma_{\eta}\left(G_{n} \oplus P\right) \uparrow \beta$ eventually stabilizes. This follows from the fact that $G_{n} \oplus P$ is admissible and is proved by induction. Assume up to $\beta$; the function taking $y<x$ to the stage at which the correct computation $\Gamma_{\eta}\left(G_{n} \oplus P, y\right)$ is defined is weakly recursive in $G_{n} \oplus P$ and so is bounded.

It follows that $\Gamma_{\eta}\left(G_{n} \oplus P\right)=Q$. As $G_{n} \oplus P$ is admissible, $Q \leqslant_{\alpha} G_{n} \oplus P$. 


\section{The Minimality Requirements}

Again this is exactly as in chapter 2. Let $\eta$ be the agent working for $M=M_{\Theta, \Phi, W}$, and let $r^{*}$ witness that $\eta$ is treated fairly.

Observation 3.28. Suppose that $s>t>r^{*}$. Suppose that at $t,(y, \pi)$ is picked as a chit for a computation $\Delta_{\eta, 0}(x)$ with use $\zeta \oplus \sigma$. Suppose that at $s,(y, \pi)$ is transferred as a chit for a $\Delta_{\eta, j}$ computation. Definitions of $\Delta$ computations are done only at $\eta$-expansionary stages, so $y<\ell(M)[t]<\ell(M)[s]$, so $y$ is $M$-confirmed at $s$. Also, $(y, \pi)$ is still active at $s$. This implies that $\phi(B \oplus W \oplus P, y)[s]=\zeta \oplus \sigma \oplus \pi$ (we use niceness of $\Phi$ here). Thus $\sigma \subset \Theta(R)[s]$. Note that once the chit is cancelled it is never active again (and will never be picked again as a new chit for $\Delta_{\eta, 0}$ as we pick large chits).

Lemma 3.29. If $\eta$ declares victory after $r^{*}$ then the hypotheses of $M$ do not hold.

Proof. If easy victory is declared then $\eta$ preserves a discrepancy between $\Theta(R)$ and $W$. Suppose that at $s>r^{*}, \eta$ enumerates a victorious chit $y$ into $Q$. Let $\zeta \oplus \sigma \oplus \pi=\phi(B \oplus W \oplus P, y)[s] . \quad \sigma \subset \Theta(R)[s]$ holds by observation 3.28 and is preserved by $\eta$ 's action at $s$ (so if $\sigma \not \subset W$ we again get an easy win.) We claim that $\zeta \oplus \pi \subset B \oplus P$ is preserved (and so that $y$ is not $M$-confirmed at $\alpha$ ). The only obstacle can be the uses of the form $\gamma_{\rho}\left(G_{n} \oplus P, y\right)$ which $\eta$ enumerates into $P$ as it declares victory. A use of this kind is enumerated only if $\Gamma_{\rho}$ clears $y$ at $s$, thus all $\gamma_{\rho}\left(G_{n} \oplus P, y\right)$ enumerated are greater than $\operatorname{dom} \pi$.

Assume that the hypotheses of $M$ hold. Then $\lim _{s \rightarrow \alpha} \ell(M)[s]=\alpha$. It follows that there are unboundedly many $M$-expansionary stages.

Let $n=n(\eta)$. 
Claim 3.30. Let $j \leqslant n$. For all $x$, there is a stage after which $\eta$ stops defining $\Delta_{\eta, j}(x)$

Proof. At each stage $s$ at which $\eta$ defines $\Delta_{\eta, j}(x)$, say with use $\zeta \oplus \sigma$, enumerate $\zeta \oplus \theta(R ; \operatorname{dom} \sigma)[s]$ into a functional $\Xi$ (recall observation 3.28). The success of the agent $\rho$ which works for $K_{\Xi}$ ensures that if unboundedly many attempts at defining $\Delta_{\eta, j}(x)$ are made, then both $\zeta \subset B$ and $\theta(R ; \operatorname{dom} \sigma)[s]$ for some such definition will be preserved. By observation 3.13, $\Delta_{\eta, j}(x)$ doesn't get redefined after $s$.

Claim 3.31. $\operatorname{dom} \Delta_{\eta, 0}(B \oplus W)=\alpha$.

Proof. By induction on $x$, we show that $\Delta_{\eta, 0}(B \oplus W, x) \downarrow$. If $x \leqslant \operatorname{dom} \Delta_{\eta, 0}(B \oplus W)$, then we know that $\Delta_{\eta, 0}(B \oplus W) \uparrow x$ eventually stabilizes. It is enough now to show that for unboundedly many $s, \Delta_{\eta, 0}(B \oplus W, x) \downarrow[s]$; by claim 3.30, after some $t, \eta$ stops defining $\Delta_{\eta, 0}(x)$, hence all computations $\Delta_{\eta, 0}(B \oplus W, x)[s]$ for $s>t$ must be the same computation, which is permanent.

Suppose that by $s^{*}>r^{*}, \Delta_{\eta, 0}(B \oplus W) \uparrow x$ is permanent. Suppose that $t>s^{*}$ and $\Delta_{\eta, 0}(W, x) \uparrow[t]$. Find some $\eta$-expansionary stage $s>t$ such that $\ell(M)[s]$ is large enough so that there is some $y \in \alpha^{[\eta]}$ such that $t<y<\ell(M)[s]$. Now if $\eta$ didn't define $\Delta_{\eta, 0}(x)$ between $t$ and $s$, then $y$ is a suitable chit (because $\eta$ did not define any $\Delta_{\eta, 0}$ computations between $t$ and $\left.s\right)$, thus $\eta$ would define $\Delta_{\eta, 0}(x)$ at $s$.

Claim 3.32. Say $j \in[1, n]$. If there are unboundedly many chits which are eventually $j-1$-suitable (and never cancelled), then $\operatorname{dom} \Delta_{\eta, j}(B \oplus W)=\alpha$.

Proof. This is like the previous claim. Letting $x, s^{*}, t$ be as above, we find an expansionary stage $s>t$ such that at $s$, there is a chit $y$ which is already $j-1$ - 
suitable and is never cancelled, such that $t<y<\ell(M)[s]$; this is possible by the assumption that there are unboundedly many such $y$.

Claim 3.33. Suppose that at stage $s, y$ is an active chit for a failed $\Delta_{\eta, j}$ computation. Suppose that $\rho \subset \eta$ works for $T_{j}$. Then $\Gamma_{\rho}$ clears $y$ at $s$.

Proof. We show that if $\Gamma_{\rho}\left(G_{j} \oplus P, y\right) \downarrow[s]$ then this computation must have been defined after the stage $t<s$ at which the chit was originally picked as a chit for a computation $\Delta_{\eta, j}(x)$. Obviously (by the size requirement for $y$ ) we have $x \leqslant y$. As the computation is failed at $s, x$ enters $G_{j}$ at a stage $u \in(t, s)$; now $x \leqslant y \leqslant \gamma_{\rho}\left(G_{j} \oplus P, y\right)[u]$ (if $\Gamma_{\rho}\left(G_{j} \oplus P, y\right) \downarrow[u]$ ), destroying the latter computation.

Lemma 3.34. There cannot be unboundedly many chits which are eventually $n$ suitable and which are never cancelled.

Proof. We show that if there are unboundedly many such chits then $\eta$ declares victory after $r^{*}$.

By assumption we can find unboundedly many $y>\operatorname{Rest}(\eta)$ and a stage $s$ at which $\eta$ is accessible, $r(\eta)[s]=0$ and $\operatorname{Rest}(\eta)[s]=\operatorname{Rest}(\eta)$, such that at $s, y$ is an active chit for a failed $\Delta_{\eta, n}$ computation and such that $y<\ell(M)[s]$. Then $y$ is victorious at $s$, and at $s, \eta$ may win by enumerating $y$ into $Q$, if $y$ is permitted promptly by $U$. The set of such $y$ s is r.e. and unbounded and so one will be permitted.

As in chapter 2, the following concludes the verifications.

Lemma 3.35. Suppose that $j \leqslant n$ and $\operatorname{dom} \Delta_{\eta, j}(B \oplus W)=\alpha$ but $\Delta_{\eta, j}(B \oplus W) \neq_{*}$ $G_{j}$. Then there are unboundedly many chits which are eventually $j$-suitable and are never cancelled. 
Proof. Much of the proof goes along classical lines. Fix $\beta<\alpha$. Let the functional $\Xi$ converge at $t>r^{*}$ with use $\zeta \oplus \rho \oplus \pi \subset B \oplus R \oplus P[t]$ if there is some $\sigma \subset W[t]$ such that $\sigma \subset \Theta(\rho)[t]$ and there is some $y>\beta$ such that at $t,(y, \pi)$ is an active chit for a failed $\Delta_{\eta, j}$ computation with use $\zeta \oplus \sigma$. If $\Xi$ converges unboundedly often, the success of $K_{\Xi}$ would show that there is some $j$-suitable chit which is never cancelled and which is greater than $\beta$ (protection of $\zeta \oplus \rho \oplus \pi \subset B \oplus R \oplus P$ ensures that $\sigma \subset W)$.

Suppose that $t^{*}>r^{*}$ is any stage. Take some $x>\beta, t^{*}$ such that $\Delta_{\eta, j}(W, x) \neq$ $Q(x)$. Suppose that the correct $\Delta_{\eta, j}(x)$ computation is defined at stage $s>t^{*}$ with associated chit $(y, \pi)$. Let $t>s$ be the stage at which this computation fails; some agent $\rho$ enumerates $x$ into $G_{j}$ at $t$. The standard argument shows that $\eta \subset \rho$; $\rho$ cannot be stronger than $\eta$ as $t>r^{*}$, and $\eta$ cannot lie to the right of $\rho$ since $\eta$ initializes nodes to its right at $s$ and $s>x$. This argument shows that $x$ is a follower for $\rho$ at $s$ and so that $\rho$ initializes weaker requirements on $\eta$ 's behalf at $s$.

Let $u$ be the least stage greater than $t$ at which $r(\eta)=0$. The key is noticing that $u$ is $M$-expansionary, as it cannot be a limit of $M$-expansionary stages. Thus $y<\ell(M)[u]$.

We verify that $\pi \subset P$ is preserved until $u$. As mentioned, $\rho$ initializes weaker agents at s. $\rho$ is not initialized until at least after $t$, as $x$ is still a follower at $t$. Thus $\pi \subset P$ is preserved between $s$ and $t$ by $\rho$ 's action. Between $t$ and $u$, restraint which is imposed by $\eta$ protects $\pi \subset P$; recall that $s$ is an $M$-expansionary stage.

Now at $u, y$ is $M$-confirmed so $\sigma \subset \Theta(R)[u]$ (note that $\sigma \subset W$ is always true since we picked the correct $\Delta_{\eta, j}(B \oplus W)$ computation). Thus $\Xi(B \oplus R \oplus P) \downarrow[u]$. 
CHAPTER 4

\section{A NEGATIVE RESULT CONCERNING EFFECTIVE SUCCESSOR MODELS}

We prove quite the opposite of the result of chapter 3 for a wide class of admissible ordinals $\alpha$.

\subsection{Preparation: Some Complexity Calculations}

Let $U$ be an amenable set. We calculate the complexities of some relations.

Let $\operatorname{Tot}(U)=\left\{e<\alpha: \Phi_{e}(U)\right.$ is total $\}$. Let $\left\langle\Phi_{e}\right\rangle$ be an effective list of all nice functionals. $\sigma \subset U$ for a string $\sigma$ is $\Delta_{0}\left(J_{\alpha}, U\right) . \sigma \subset \Phi_{e}(U)$ is $\Sigma_{1}\left(J_{\alpha}, U\right)$. $\operatorname{Tot}(U)$ is $\Pi_{2}\left(J_{\alpha}, U\right)$. Note that $\left\{\Phi_{e}(U): e \in \operatorname{Tot}(U)\right\}$ only varies over all amenable sets recursive in $U$; we don't mind as we only need r.e. degrees.

A $U$-index is an element of $\operatorname{Tot}(U)$. For shorthand, we write $X_{e}$ instead of $\Phi_{e}(U)$ for all $e \in \operatorname{Tot}(U)$. We consider the following relations among $U$-indices:

$\Phi_{e}\left(X_{a}\right)=X_{b}$ is $\Pi_{2}\left(J_{\alpha}, U\right)$ because $\sigma \subset \Phi_{e}\left(\Phi_{i}(U)\right)$ is $\Sigma_{1}\left(J_{\alpha}, U\right)$. Thus $X_{a} \leqslant \alpha X_{b}$ and $X_{a} \equiv_{\alpha} X_{b}$ are $\Sigma_{3}\left(J_{\alpha}, U\right)$.

$X_{a}=W_{e}$ is $\Pi_{2}\left(J_{\alpha}, U\right)$. Thus $X_{a} \equiv_{\alpha} W_{e}$ and " $X_{a}$ has r.e. degree" are $\Sigma_{3}\left(J_{\alpha}, U\right)$.

There is a recursive function $\mathrm{j}$ oin such that for all $a, b \in \operatorname{Tot}(U), X_{\text {join }(a, b)}=$ $X_{a} \oplus X_{b}$

Lemma 4.1. Suppose that $A(x, y)$ is $\Sigma_{n}\left(J_{\alpha}, U\right)(n>0)$. Then the formula

"t is finite \& $\forall x \in t A(x, y)$ "

is also $\Sigma_{n}\left(J_{\alpha}, U\right)$. 
Proof. We first prove this for $n=1$. This is fairly easy. Suppose that $C$ is a bounded formula such that $A(x, y) \Leftrightarrow \exists z C(x, y, z)$. Then the required formula is equivalent to

$$
\text { " } t \text { is finite \& } \exists u \forall x \in t \exists z \in u C(x, y, z) \text { "; }
$$

this is because $J_{\alpha}$ is closed under taking finite subsets.

Suppose by induction that the statement holds for $n$. Let $A(x, y)$ be a formula which is $\Sigma_{n+1}\left(J_{\alpha}, U\right)$ and let $C$ be the $\Pi_{n}\left(J_{\alpha}, U\right)$ formula such that for all $x$ and $y, A(x, y) \leftrightarrow \exists z C(x, y, z)$. Let $\psi(x, y, u)$ be the formula

"if $u$ is finite then $\exists z \in u C(x, y, z)$ ".

By the induction hypothesis, $\psi$ is $\Pi_{n}\left(J_{\alpha}, U\right)$. By quantifier contraction,

$$
\forall x \in t \psi(x, y, u)
$$

is also $\Pi_{n}\left(J_{\alpha}, U\right)$. Again because $J_{\alpha}$ is closed under taking finite subsets, the required formula is equivalent to

" $t$ is finite \& $\exists u(u$ is finite \& $\forall x \in t \psi(x, y, u)) "$

which is easily seen to be $\Sigma_{n+1}\left(J_{\alpha}, U\right)$.

\subsection{More on Effective Models}

Recall that the correctness condition $\chi_{\text {effective }}(\overline{\mathbf{p}}, \overline{\mathbf{e}})$, defined in the beginning of chapter 3 states that $\overline{\mathbf{p}}$ codes an effective successor model of arithmetic, witnessed by $\overline{\mathbf{e}}$. We note that if $\chi_{\text {effective }}(\overline{\mathbf{p}}, \overline{\mathbf{e}})$ holds, then the function taking $\mathbf{x} \in M_{\overline{\mathbf{p}}}$ to $\sum_{\overline{\mathbf{p}}} \mathbf{x}$ is definable from $\overline{\mathbf{p}}$ (uniformly in such $\overline{\mathbf{p}}$ ). 
Fix $\overline{\mathbf{p}}, \overline{\mathbf{e}}$ satisfying $\chi_{\text {effective }}$. Let $U$ be an amenable r.e. set which computes all the parameters $\overline{\mathbf{p}}, \overline{\mathbf{e}}$. Using the notation of the first subsection, consider the following relations.

- " $X_{a} \in 0^{M_{\overline{\mathbf{p}}}}$ " is $\Sigma_{3}\left(J_{\alpha}, U\right)$ (as we can use a particular $U$-index for an element of $\left.0^{M_{\overline{\mathbf{p}}}}\right)$.

- There is a $\Sigma_{3}\left(J_{\alpha}, U\right)$ relation $S$ such that for all $a$, if $\operatorname{deg}\left(X_{a}\right) \in M_{\overline{\mathbf{p}}}$ then for all $b \in \operatorname{Tot}(U), S(a, b)$ holds iff $\operatorname{deg}\left(X_{b}\right)=\left(\operatorname{deg}\left(X_{a}\right)+1\right)^{M_{\overline{\mathbf{p}}}} . \quad S$ is the following relation:

$X_{b} \geqslant_{\alpha} \mathbf{b} \& X_{b} \leqslant_{\alpha} \mathbf{r} \& \mathbf{q} \leqslant \alpha X_{b} \vee \mathbf{p} \& \bigvee_{i<2}\left(X_{b} \leqslant_{\alpha} X_{a} \vee \mathbf{e}_{i} \& X_{b} \leqslant \alpha \mathbf{f}_{i}\right)$

- " $X_{a} \in n^{M_{\overline{\mathrm{p}}}}$ " (for $\left.n<\omega\right)$ is $\Sigma_{3}\left(J_{\alpha}, U\right)$. It holds iff there is a string $\sigma$ of length $n+1$ such that $X_{\sigma(0)} \in 0^{M_{\overline{\mathbf{p}}}}$ and for all $i<n, S(\sigma(i), \sigma(i+1))$. This uses lemma 4.1.

We now add our assumption on $U$ and $\alpha$ which is: every $\Sigma_{3}\left(J_{\alpha}, U\right)$ relation with domain $\omega$ is uniformized by some $\alpha$-finite function.

Let $\psi(n,(a, e))$ be the relation

$$
" a \in \operatorname{Tot}(U) \quad \& \quad X_{a} \in n^{M_{\overline{\mathbf{p}}}} \& X_{a}=W_{e} "
$$

$\psi$ is $\Sigma_{3}\left(J_{\alpha}, U\right)$. By assumption, there is an $\alpha$-finite function $f: \omega \rightarrow \alpha^{2}$ which uniformizes $\psi$. Let

$$
C=\bigoplus_{n<\omega} W_{(f(n))_{1}}=\bigoplus_{n<\omega} X_{(f(n))_{0}} .
$$

$C$ is r.e. and recursive in $U$.

Lemma 4.2. In the r.e. degrees below $U, C$ is the least upper bound for the standard part of $M_{\overline{\mathbf{p}}}\left(\left\{n^{M_{\overline{\mathbf{p}}}}: n<\omega\right\}\right)$. 
Proof. Suppose that $Y \leqslant_{\alpha} U$ is amenable and that for all $n, n^{M_{\overline{\mathbf{p}}}} \leqslant_{\alpha} Y$. Let $R(n, e)$ be the relation $\Phi_{e}(Y)=X_{\left(f(n)_{1}\right)}$. This is $\Sigma_{3}\left(J_{\alpha}, U\right)$; again by assumption we get an $\alpha$-finite function $g: \omega \rightarrow \alpha$ uniformizing $R$; $g$ gives us a way to uniformly compute $C$ from $Y$.

Lemma 4.3. If $\mathbf{x}$ belongs to the nonstandard part of $M_{\overline{\mathbf{p}}}$ then $\sum_{\overline{\mathbf{p}}} \mathbf{x} \Varangle_{\alpha} C$.

Proof. For every nonstandard $\mathbf{y} \in M_{\overline{\mathbf{p}}}$ and every $n<\omega, n^{M_{\overline{\mathbf{p}}}} \leqslant \sum_{\overline{\mathbf{p}}} \mathbf{y}$ and so $C \leqslant \alpha \sum_{\overline{\mathbf{p}}} \mathbf{y}$. Suppose that $\mathbf{x} \in M_{\overline{\mathbf{p}}}$ is nonstandard and assume for contradiction that $\sum_{\overline{\mathbf{p}}} \mathbf{x} \leqslant{ }_{\alpha} C$, so we have $\sum_{\overline{\mathbf{p}}} \mathbf{x} \equiv_{\alpha} C . \quad \mathbf{x}$ is nonstandard so there is some $\mathbf{y}<{ }^{M_{\overline{\mathbf{p}}}} \mathbf{x}$ which is also nonstandard; $\sum_{\overline{\mathbf{p}}} \mathbf{y} \leqslant \sum_{\overline{\mathbf{p}}} \mathbf{x}$ so we have $\sum_{\overline{\mathbf{p}}} \mathbf{y}=\sum_{\overline{\mathbf{p}}} \mathbf{x}$. This is impossible because $\left\langle\sum_{\overline{\mathbf{p}}} \mathbf{y}: \mathbf{y} \in M_{\overline{\mathbf{p}}}\right\rangle$ is strictly increasing with $<^{M_{\overline{\mathbf{p}}}}$ (for example, $\mathbf{y} \leqslant \sum_{\overline{\mathbf{p}}} \mathbf{x}$ and $\mathbf{y} \nless \sum_{\overline{\mathbf{p}}} \mathbf{y}$.) This is a contradiction.

Let $\theta(x, \mathbf{c}, \overline{\mathbf{p}})$ state that $x \in M_{\overline{\mathbf{p}}}$ and $\sum_{\overline{\mathbf{p}}} x \leqslant \mathbf{c}$. We thus see that $\theta\left(\mathcal{R}_{\alpha}, \mathbf{c}, \overline{\mathbf{p}}\right)$ is the standard part of $M_{\overline{\mathbf{p}}}$. We have shown:

Theorem 4.4. Suppose that $\alpha$ is admissible $U$ is an amenable r.e. set and that every $\Sigma_{3}\left(J_{\alpha}, U\right)$ relation with domain $\omega$ is uniformized by an $\alpha$-finite function. Then for all $\overline{\mathbf{p}}, \overline{\mathbf{e}}$ below $U$ satisfying $\chi_{\text {effective }}$, there is some $\mathbf{c} \leqslant_{\alpha} U$ which is the least upper bound in the degrees below $U$ of the standard part of $M_{\overline{\mathbf{p}}}$ and moreover that standard part is definable as $\theta\left(\mathcal{R}_{\alpha}, \mathbf{c}, \overline{\mathbf{p}}\right)$.

We remark again that for the $\alpha$ s discussed in chapter 3, the models constructed show that the above statement does not hold for any promptly simple $U$.

\subsection{Examples of $\alpha$ and $U$}

We give three examples for pairs $\alpha$ and $U$ satisfying the above assumption. In all cases we of course must have $\operatorname{cf}_{\Sigma_{3}\left(J_{\alpha}\right)}(\alpha)>\omega$. 


\subsection{1 $\varrho_{\alpha}^{2}=\alpha$ and $U$ is low}

In this case, $\Sigma_{3}\left(J_{\alpha}, U\right)=\Sigma_{3}\left(J_{\alpha}\right)$, and every $\Sigma_{3}\left(J_{\alpha}\right)$ function from $\omega$ to $\alpha$ is $\alpha$-finite.

Let $C$ be a $\Sigma_{3}\left(J_{\alpha}, U\right)$-relation with domain $\omega$. $U$ is low so $U$ is admissible and so $\Sigma_{2}\left(J_{\alpha}, U\right)$ is the same as r.e. in $U^{\prime}$ which is the same as r.e. in $0^{\prime}$ which is the same as $\Sigma_{2}$. By Jensen's uniformization theorem, $C$ can be uniformized by some $\Sigma_{3}$ function $f: \omega \rightarrow \alpha$.

Let $A$ be a $\Pi_{2}\left(J_{\alpha}\right)$ relation such that $f(n)=\gamma$ iff $\exists z A(n, \gamma, z)$. Consider $A$ as a relation between $n$ and $(\gamma, z)$; by Jensen's uniformization theorem there is a function $g$ which is $\Sigma_{3}\left(J_{\alpha}\right)$ and uniformizes $A$. As by assumption $\mathrm{cf}_{\Sigma_{3}\left(J_{\alpha}\right)}(\alpha)>\omega$, it follows that there is some $\alpha$-finite $\beta>$ sup range $f$ such that for all $n<\omega$ there is some $z<\beta$ such that $A(n, f(n), z)$ holds. $A \cap\left(\omega \times \beta^{2}\right)$ is $\alpha$-finite by assumption, and $f$ can be effectively defined by that set.

\subsection{2 $\alpha$ is $\Sigma_{2}$-admissible and $U$ is low $_{2}$}

In this case too we have $\Sigma_{3}\left(J_{\alpha}, U\right)=\Sigma_{3}\left(J_{\alpha}\right)$ and every $\Sigma_{3}\left(J_{\alpha}\right)$ function from $\omega$ to $\alpha$ is $\alpha$-finite.

Let $V=U^{\prime}$. We work in the admissible structure $M=\left(J_{\alpha}, 0^{\prime}\right) . V$ is low in the $M$-degrees; again we get that $\Sigma_{2}(M, V)=\Sigma_{2}(M)$. But $\Sigma_{2}(M)=\Sigma_{3}\left(J_{\alpha}\right)$ and $\Sigma_{2}(M, V)=\Sigma_{3}\left(J_{\alpha}, U\right)$ because $\Sigma_{2}\left(J_{\alpha}, U\right)=\Sigma_{1}\left(J_{\alpha}, V\right)$ (by $U$ 's admissibility) and the latter is the same as $\Sigma_{1}(M, V)$ as $0^{\prime} \leqslant \leqslant_{\alpha} V$.

Let $C$ be a $\Sigma_{3}\left(J_{\alpha}, U\right)$ relation with domain $\omega$. So $C$ is $\Sigma_{3}\left(J_{\alpha}\right)$ and so can be uniformized by some $\Sigma_{3}\left(J_{\alpha}\right)$ function $f: \omega \rightarrow \alpha$.

Again work in $M . f$ is $\Sigma_{2}(M)$. Given any $f: \omega \rightarrow \alpha$ which is $\Sigma_{2}(M)$ (and so actually $\Delta_{2}(M)$, we get an $M$-recursive approximation $f[s]$ to $f$; by the fact that $\left.\operatorname{cf}_{\Sigma_{2}(M)}\right)(\alpha)>\omega$ we know that the approximation has to stabilize by some $\alpha$-finite 
stage, so $f[s]=f$ for some $s<\alpha$; by admissibility of $M, f[s] \in J_{\alpha}$.

\subsection{3 $\alpha$ is $\Sigma_{3}$-admissible, $\operatorname{cf}_{\Sigma_{4}\left(J_{\alpha}\right)}(\alpha)>\omega$ and $U$ is in any r.e. degree}

In this case every $\Sigma_{3}\left(J_{\alpha}, U\right)$ relation is $\Sigma_{4}\left(J_{\alpha}\right)$ relation and so can be uniformized by a $\Sigma_{4}\left(J_{\alpha}\right)$ function. Now if $f: \omega \rightarrow \alpha$ is $\Sigma_{4}\left(J_{\alpha}\right)$ then it is $\Sigma_{2}(M)$ where $M=\left(J_{\alpha}, 0^{\prime \prime}\right)$ is admissible and as in the previous subsection we get that $f$ is $\alpha$-finite.

\subsection{4 $\varrho_{\alpha}^{3}=\alpha, \operatorname{cf}_{\Sigma_{4}\left(J_{\alpha}\right)}(\alpha)>\omega$ and $U$ is in any r.e. degree}

Again, $\Sigma_{3}\left(J_{\alpha}, U\right)$ is $\Sigma_{4}$ and every $\Sigma_{4}$ function $f: \omega \rightarrow \alpha$ is $\alpha$-finite; this is as in subsection 4.3.1. 


\section{CHAPTER 5}

\section{A NONEMBEDDING RESULT}

We show that lattices with critical triples (for example, the 1-3-1) cannot be embedded below a degree which cannot compute a cofinal $\omega$-sequence in $\alpha$.

Definition. Let $A \subset \alpha$ be amenable and let $\Psi$ be a nice functional. $\gamma<\operatorname{dom} \Psi(A)$ is a $\Psi, A$-closure point if for all $\beta<\gamma, \psi(A ; \beta)<\gamma$.

Proposition 5.1. Let $A$ be amenable, $\Psi$ a nice functional, and suppose that $\operatorname{dom} \Psi(A)=\alpha$. Also assume that $\operatorname{rcf}(A)>\omega$. Then the collection of $\Psi, A$-closure points is a club of $\alpha$ which is recursive in $A$.

Proof. It is immediate from the definition that the collection of closure points is closed. The function $\gamma \rightarrow \psi(A ; \gamma)$ is weakly recursive in $A$. For all $\gamma<\alpha$, the sequence $\left\langle\gamma_{n}\right\rangle_{n<\omega}$ defined inductively by $\gamma_{0}=\gamma, \gamma_{n+1}=\psi\left(A ; \gamma_{n}+1\right)$ is strictly increasing and weakly recursive in $A$; hence, bounded below $\alpha$. Its supremum is a $\Psi, A$-closure point.

Since we'd like to get points which are both $\Psi_{0}, A$ - and $\Psi_{1}, A$-closure points, we mention

Proposition 5.2. Suppose that $C, D$ are clubs of $\alpha$ and that $\operatorname{rcf}(C \oplus D)>\omega$. then $C \cap D$ is a club.

If $A$ is r.e., then we can guess, at some stage $s<\alpha$, whether some $\gamma$ is a closure point: we say that $\gamma<\operatorname{dom} \Psi(A)[s]$ is a $\Psi, A$-closure point at $s$ if for all $\beta<\gamma$, $\psi(A ; \beta)[s]<\gamma$. 
Observation 5.3. Suppose $A$ is r.e. and amenable, and that $\gamma$ is a $\Psi, A$-closure point. There is some $s<\alpha$ such that $A \uparrow \psi(A ; \gamma)=A[s] \uparrow \psi(A ; \gamma)$. Then for all $t>s, \gamma$ is a $\Psi, A$-closure point at $t$, detected by the correct computations.

Definition (Weinstein [Wei88]). Suppose $L$ is an upper semi-lattice. A weak critical triple in $L$ is a triple $a_{0}, a_{1}, b$ such that $a_{0} \vee b=a_{1} \vee b, a_{0} \nless b$ and there is no $e \leqslant a_{0}, a_{1}$ such that $a_{0} \leqslant e \vee b$.

Suppose that $A_{0}, A_{1}$ and $B$ are amenable and r.e., and that $A_{0} \oplus B \equiv_{\alpha} A_{1} \oplus$ $B$. Then there are nice functionals $\Psi_{0}$ and $\Psi_{1}$ such that $\Psi_{0}\left(B \oplus A_{1}\right)=A_{0}$ and $\Psi_{1}\left(B \oplus A_{0}\right)=A_{1}$. Let $A=B \oplus A_{0} \oplus A_{1}$. Assume that $\operatorname{rcf}(A)>\omega$.

We construct an r.e. set $E$. For all $\gamma<\alpha$ and $s<\alpha$ we'll have $E^{[\gamma]}[s] \in \alpha$. The instructions are: Suppose that at stage $s, \gamma$ is both a $\Psi_{0}, B \oplus A_{0}$ and a $\Psi_{1}, B \oplus A_{1}$-closure point, and that a change in $A_{0}$ or $A_{1}$ is detected below $\gamma$ (that is, $A_{i} \uparrow \gamma[s] \neq A_{i} \uparrow \gamma[t]$ for all $t<s$ for $i$ either 0 or 1.) It follows that either $B \uparrow \gamma[s] \neq B \uparrow \gamma$ or $A_{1-i} \uparrow \gamma[s] \neq A_{1-i} \uparrow \gamma$. We can effectively find some stage $t>s$ at which some $x<\gamma$ enters either $B$ or $A_{1-i}$.

Say that we found a change at $A_{1-i}$ below $\gamma$. We then enumerate $E^{[\gamma]}[s]$ into $E^{[\gamma]}$ at $s$.

By permitting (and the fact that $\gamma \leqslant\langle\gamma, i\rangle$ for any $i$ ), $E \leqslant{ }_{\alpha} A_{0}, A_{1}$.

We give an algorithm to compute both $A_{i}$ from $E \oplus B$. At stage $s$, suppose that $\gamma$ is both a $\Psi_{0}, B \oplus A_{0}$ and a $\Psi_{1}, B \oplus A_{1}$-closure point and that $B \uparrow \gamma[s]$ is correct. Then $A_{i} \uparrow \gamma[s]$ are correct iff $E^{[\gamma]}[s] \notin E^{[\gamma]}$. All of $A_{i}$ can be thus computed, because there are unboundedly many $\gamma$ which are both $\Psi_{0}, B \oplus A_{0}$ and $\Psi_{1}, B \oplus A_{1}$-closure points (and that fact is eventually approximated, witnessed by the correct computations). 
Thus we can conclude:

Theorem 5.4. If $\operatorname{rcf}(\mathbf{a})>\omega$ then in the $\alpha$-r.e. degrees, there is no weak critical triple below a. 
CHAPTER 6

\section{EMBEDDING THE 1-3-1 LATTICE}

This chapter complements chapter 5 by proving:

Theorem 6.1. If $D$ is a collapsible r.e. set, then there is an embedding of the 1-3-1 lattice in the $\alpha$-r.e. degrees below D.

For the notion of a collapsible set (or degree) see appendix D.

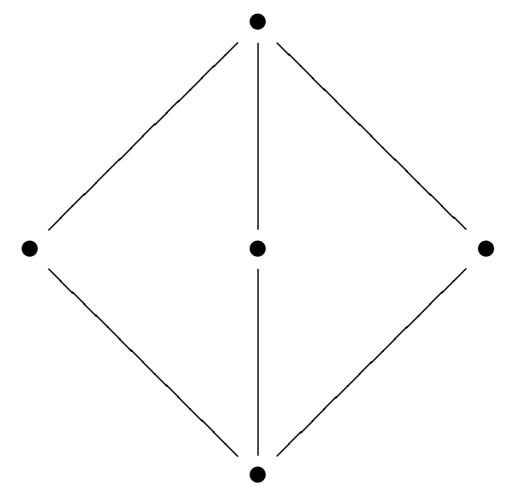

Figure 6.1: The 1-3-1 lattice

For the construction, we use ideas for working below nonlow 2 degrees from [DS96] and Fejer's construction of a branching degree ([Fej82]).

\subsection{Preparation}

Let $D$ be an amenable r.e. set such that there is some bijection $p: \omega \leftrightarrow \alpha$, weakly recursive in $D$.

Lemma 6.2. There is a recursive approximation $p[s]$ for $p$ with the following properties:

- For every s there is some $n<\omega$ such that $\neg\left(p(n)[s]=\lim _{t \rightarrow s} p(n)[t]\right)$. 
- There is an increasing sequence $\left\langle\beta_{n}\right\rangle$, weakly recursive in $D$, such that after $\beta_{n}, p\lceil n[s]$ is permanent.

Proof. Let $p_{0}[s]$ be a recursive approximation for $p$ which we get by approximating $p$ 's computation from $D$. Define $p[s]$ by induction on $s$. If $\lim _{t \rightarrow s} p(n)[t]$ does not exist for some $n<\omega$ then let $p[s]=p_{0}[s]$. Otherwise, let $p[s]$ be $p_{0}[u]$ for the least $u>s$ such that for some $n, p_{0}(n)[u] \neq \lim _{t \rightarrow s} p(n)[t]$. If $p_{0}\lceil n[s]$ is correct then so is $p\lceil n$.

$\left\langle\beta_{n}\right\rangle$ is necessarily cofinal in $\alpha$. For $s<\omega$ we let $n(s)$ be the least $n$ such that either $\lim _{t \rightarrow s} p(n)[t]$ does not exist, or exists and is not equal to $p(n)[s]$. For all $s \geqslant \beta_{k}$ we have $n(s) \geqslant k$.

Proposition 6.3. For every $f: \omega \rightarrow \alpha$ which is weakly recursive in $0^{\prime}$, there is some $g: \omega \rightarrow \alpha$, weakly recursive in $D$, which is not dominated by $f$, i.e.

$$
\{n<\omega: g(n) \geqslant f(n)\}
$$

is infinite.

Proof. By Shore ([Sho76b] $], D^{\prime} \equiv_{\alpha} 0^{\prime \prime}$.

Consider the admissible collapse above $D$ (see appendix D). Let $\mathcal{D}$ be $D$ 's collapse and let $\mathcal{K} \geqslant_{T} \mathcal{D}$ be $0^{\prime \prime}$ s collapse. Theorem D.11 implies that $\mathcal{K}^{\prime \prime} \equiv_{T} \mathcal{D}^{\prime \prime}$. In other words, $\mathcal{K} \in \mathbf{L}_{2}(\mathcal{D})$. In particular, $\mathcal{K}$ is not high over $\mathcal{D}$. Thus, for every $f \leqslant_{T} \mathcal{K}$ there is some $g \leqslant_{T} \mathcal{D}$ which is not dominated by $f$.

By the definition of the admissible collapse, we have, for every $f: \omega \rightarrow \omega$ which is weakly recursive in $0^{\prime}$, there is some $g: \omega \rightarrow \omega$, not dominated by $f$, which is weakly recursive in $D$. 
Let $f: \omega \rightarrow \alpha$ be weakly recursive in $0^{\prime}$. For $k<\omega$ let $\tilde{f}(k)$ be the least $n$ such that $f(k)<\beta_{n}$. $\tilde{f}$ is weakly recursive in $0^{\prime}$, so there is some $\tilde{g}: \omega \rightarrow \omega$, weakly recursive in $D$, which is not dominated by $\tilde{f}$. Let $g(k)=\beta_{\tilde{g}(k)} ; g$ is as required.

Proposition 6.4. There is a function $f: \omega \rightarrow \alpha$, weakly recursive in $0^{\prime}$, such that for every r.e. set $W$, if for all $n<\omega, W^{[n]} \backslash \beta_{n}$ is finite, then for all but finitely $\operatorname{many} n<\omega, f(n)>\max W^{[n]}$.

Proof. The set $\left\{e: W_{e}\right.$ is infinite $\}$ is r.e. This is because every infinite r.e. set $W$ contains an infinite, $\alpha$-finite r.e. set, which is enumerated into $W$ by an $\alpha$-finite stage. Thus this set is recursive in $0^{\prime}$. Given $e$ such that $W_{e}$ is finite, $0^{\prime}$ can compute $\max W_{e}$ (that is to say, the function $e \rightarrow \max W_{e}$, defined on all $e$ such that $W_{e}$ is finite, is weakly recursive in $0^{\prime}$.)

Thus the function $m(e, n)$, which returns $\max W_{e}^{[n]} \backslash \beta_{n}$ if $W_{e}^{[n]} \backslash \beta_{n}$ is finite and nonempty, and $\beta_{n}$ otherwise, is weakly recursive in $0^{\prime}$. So we can let

$$
f(n)=\max \{m(p(k), l): k, l \leqslant n\}
$$

Let $g: \omega \rightarrow \alpha$ be weakly recursive in $D$, which is not dominated by $f$ (of proposition 6.4). Let $g[s]$ be a recursive approximation for $g$ which we get from approximating $g$ 's computation from $D$. For every $n, D$ can effectively find a stage after which $g\lceil n[s]$ is permanent. We may assume that sup range $g[s] \leqslant s$.

\subsection{The Embedding}

We construct $A_{0}, A_{1}, A_{2}$ and $B$ fulfilling the requirements:

$P: \operatorname{otp} \neg B=\omega$.

$H_{\Psi, i}: \Psi(B) \neq A_{i}$ 
$G_{\Phi, i, j}:$ If $i \neq j$ and $\Phi\left(B \oplus A_{i}\right)=\Phi\left(B \oplus A_{j}\right)=C$ then $C \leqslant{ }_{\alpha} B$.

and such that $A_{i} \leqslant{ }_{\alpha} B \oplus A_{j} \oplus A_{k}$, and $B, A_{i} \leqslant{ }_{\alpha} D$.

\subsubsection{Elements of the Construction}

All functionals given are nice. At stage $s<\alpha$, we enumerate $\neg B$ as $\left\langle b_{i}[s]\right\rangle_{i<\alpha}$ (At the end of the construction, enumerate $\neg B$ as $\left\langle b_{n}\right\rangle_{n<\omega}$.) As we expect to have otp $\neg B=\omega$, we only consider computations which mention finitely much negative information about $B$. That is, we only regard computation with use bounded below $b_{\omega}[s]$.

Whenever a number $b_{n}[s]$ enters $B$, we also enumerate all of $\left\{b_{m}[s]: m>n\right\}$ into $B$; we may do this because we just realized that our guess about the value for each such $b_{m}$ was incorrect.

We order $H$ and $G$ requirements effectively in order-type $\alpha$. The tree of strategies will be $2^{<\omega}$. At stage $s$, we declare that agents of level $n$ work for requirement $p(n)[s]$.

Notation. Throughout, we denote a node working for an $H$ requirement by the letter $\eta$ (and sometimes call it a "hole"). A node working for a $G$ requirement is called a "gate" and is denoted by $\rho$.

Convention. At the beginning of stage $s$, all numbers involved in the construction are below $\omega s$. At stage $s$ we add finitely many elements to the construction (balls, markers) which are "large", these will be chosen from $(\omega s, \omega(s+1))$; this will ensure this convention holds at $s+1$. Also note that at stage $s$ we do not choose $\omega s$ to be any new ball or marker; this is not very significant but will help us show that otp $\neg B \geqslant \omega$. 


\section{The basic strategy for $P$}

$P$ is a global "dumping" requirement. At every stage $s, P$ enumerates all but finitely many numbers $<\omega s$ into $B$.

\section{The basic strategy for $H$}

Holes and Gates follow Lachlan's ideas for embedding the 1-3-1 in the $\omega$-r.e. degrees, modified to use Fejer's method of constructing branching degrees. The construction is a pinball machine on the tree of agents; all followers and traces are balls on the machine, which can reside at various nodes.

A hole $\eta$ working for $H_{\Psi, i}$ appoints a follower $x$ and waits for it to be realized, i.e. for $\Psi(B, x) \downarrow=0$. Positive order requirements are met by a tracing procedure. Any ball $y_{0}$ targeted for $A_{i}$ is appointed a trace $y_{1}$ which is targeted for either $A_{j}$ or $A_{k}$, where $\{i, j, k\}=3$. The trace $y_{1}$ receives a trace of its own, a stage after being appointed. As in chapter 2, the collection of balls consisting of a follower and closed under taking traces, is called an entouage.

Once a follower $x$ for $\eta$ is released from its hole, its entire entourage moves down the tree, to the next $\eta$-gate. An $\eta$-gate is a gate $\rho$ such that $\rho\urcorner 0 \subset \eta$ (here 0 denotes the infinitary outcome). At the next stage at which $\eta$ is accessible, the $\rho$-permissible tail of $x$ 's entourage is released from $\rho$ and descends to the next $\eta$-gate. The $\rho$-permissible tail is the longest final segment of the entourage which does not contain both a ball targeted for $A_{i}$ and a ball targeted for $A_{j}$, where $\rho$ works for $G_{\Phi, i, j}$. This procedure repeats itself; each time $\eta$ is accessible, there is a final segment of $x$ 's entourage, waiting at some $\eta$-gate $\rho$ (the rest of the entourage lies at higher gates), and the $\rho$-permissible tail descends further to the next $\eta$-gate.

An entourage tail which is released from the lowest $\eta$-gate is placed in the 
permitting bin. After balls in the tail are permitted to enter the sets for which they are targeted, the new tail of the entourage is residing at some $\eta$-gate $\rho$, and the process repeats itself.

Once a follower $x$ is enumerated into its target set $A_{i}, \eta$ declares victory and ceases all action (until the next time it is initialized).

This orderly picture relies upon the fact that there is always a nonempty $\rho$ permissible tail; this is ensured by the entourage being finite. Thus at the beginning of every stage we winnow out entourages and make them all finite. This winnowing is coded into $B$, and so does not interfere with the tracing procedure.

When appointing new traces to the end of an entourage residing at a gate $\rho$, we target the new traces such that the $\rho$-permissible tail of the entourage does not get shorter. This implies that whenever some tail of the entourage leaves $\rho$, at least one ball in that tail originated above $\rho$ and arrived at $\rho$ at some point, and was not appointed as a trace at $\rho$. We thus have

Lemma 6.5. For any follower $x$ there are only finitely many stages at which balls from $x$ 's entourage move.

\section{The basic strategy for $G$}

A gate $\rho$ builds a functional $\Gamma_{\rho}$ with intended oracle $B$. The intention is that $\Gamma_{\rho}(B, n)=\Phi\left(B \oplus A_{i} ; b_{n}\right)$, so if there is agreement between $\Phi\left(B \oplus A_{i} ; b_{n}\right)$ and $\Phi\left(B \oplus A_{j} ; b_{n}\right)$ at a certain stage, $\rho$ would like to set this common value as $\Gamma_{\rho}(B, n)$. When $\rho$ defines $\Gamma_{\rho}(B, n)$, the use will always be $\left(B \uparrow b_{m}+1\right)[s]$ for some $m>n$. That number $b_{m}[s]$ is called the marker for this computation and is (sloppily) denoted by $\gamma_{\rho}(n)$. When instructed to remove the computation $\Gamma_{\rho}(B, n)[s], \rho$ enumerates $\gamma_{\rho}(n)[s]$ into $B$ (in this way $\rho$ will be able to remove computations 
that are seen to be incorrect). Of course we also require that $\gamma_{\rho}(n+1)>\gamma_{\rho}(n)$ so $\operatorname{dom} \Gamma_{\rho}(B)[s]$ is closed downwards; at $s$ we only define $\Gamma_{\rho}\left(B, \operatorname{dom} \Gamma_{\rho}(B)[s]\right)$.

Definition. If $\rho$ works for $G_{\Phi, i, j}$, then a computation $\Gamma_{\rho}(B, n)=\sigma$ is semibelievable (at stage $s$ ) if $\operatorname{dom} \sigma=b_{n}[s]$ [if a computation is not even semi-believable, then it has no hope.] It is l-believable (for $l \in\{i, j\}$ ) if it is semi-believable and $\Phi\left(B \oplus A_{l} ; b_{n}\right)=\sigma[s]$. It is believable if it is either $i$-believable or $j$-believable; it is doubly believable if it is both $i$ - and $j$-believable.

At every stage, after every time numbers enter sets, all gates $\rho$ on the tree examine their $\Gamma_{\rho}(B)$ and search for computations which are not believable ("unbelievable" computations). If one is found, it is to be removed.

While removing computations, numbers go into $B$, rendering even more computations unbelievable. These too will have to be removed immediately. And so on, until only believable computations are left. This process is called cascading.

To maintain order, when a gate $\rho$ acts and removes computations, it initializes all nodes to the right of $\rho^{\frown} 0 . \rho$ does not initialize nodes which extend $\rho^{\frown} 0$, Since if the correct outcome is 0 (agreement), $\rho$ will remove computations unboundedly often.

We remark that the dumping requirement ensures that after acting, for every gate $\rho, \operatorname{dom} \Gamma_{\rho}(B)[s]$ is finite.

\section{Permission}

It may be that a tail of an entourage of a follower for hole $\eta$ will wait in the permitting bin eternally. To counter this, as usual, $\eta$ will appoint more followers. When all followers have entourage tails in the permitting bin, $\eta$ appoints a new 
follower. The dumping requirement $P$ will ensure that at every stage, a hole has only finitely many followers on the machine.

The priority ordering between followers is determined by date of birth (which is the same as by size, as we appoint large followers).

We say that a number $m$ is permitted at $s$ if for some $k \leqslant m$,

$$
\neg\left(g(k)[s]=\lim _{t \rightarrow s} g(k)[t]\right)
$$

(in particular if the limit does not exist.) A tail of the entourage for the $m^{\text {th }}$ follower (in strength) of $\eta$ is permitted at $s$ exactly when $m$ is permitted. Note that when permission is obtained, the balls must be enumerated immediately, even if $\eta$ is not accessible.

\section{Initialization}

We explained when gates initialize other nodes. Holes also initialize other nodes; whenever a hole, or one of its followers, receives attention (which includes appointing a follower, some part of an entourage of a follower moves or gets enumerated, or some other special delicacy I'm reserving for later), the hole initializes all weaker nodes: nodes above and to the right (this kind of action is finitary).

Also as usual, the accessible nodes initialize nodes to the right.

Now what happens when a node is initialized? If it is a hole, then all of its followers are removed from the tree, and if it declared victory before, it is restored to the hungry state of dissatisfaction. If it is a gate, then all $\Gamma$ computations are cancelled and the gate needs to define them afresh. 


\section{Accessible nodes}

Accessible nodes are defined at each stage very much as is the custom for tree constructions. For the $G_{\Phi, i, j}$ requirement, define

$$
\ell(\Phi, i, j)[s]=\max \left\{\beta: \Phi\left(B \oplus A_{i} ; \beta\right) \downarrow=\Phi\left(B \oplus A_{j} ; \beta\right) \downarrow[s]\right\} .
$$

We say that $\rho$ is expansionary at $s$ if it is accessible at $s$, and if for all $t<s$ at which $\rho$ was accessible, $\ell(\Phi, i, j)[s]>\ell(\Phi, i, j)[t]$.

For future reference we note

Lemma 6.6. If $\rho$ is expansionary at $s$ then $\Gamma_{\rho}(B)[s]$ is doubly believable.

Proof. Unbelievable computations are removed, hence $\Gamma_{\rho}(B)[s]$ is believable. Let

$$
m+1=\operatorname{dom} \Gamma_{\rho}(B)[s]
$$

and say

$$
\Phi\left(B \oplus A_{i} ; b_{m}\right) \downarrow[s]=\Gamma_{\rho}(B, m)[s]=\sigma .
$$

Now the computation $\Gamma_{\rho}(B, m)=\sigma$ was enumerated into $\Gamma_{\rho}$ by $\rho$ at an earlier stage $t<s$, at which $\rho$ was accessible. Now $b_{m}[t]=b_{m}[s]$ (or the computation becomes unbelievable). $\rho$ defines a $\Gamma$ computation only if there is agreement between $A_{i}$ and $A_{j}$; thus $\ell(\Phi, i, j)[t] \geqslant b_{m}[s]$. Thus $\ell(\Phi, i, j)[s]>b_{m}[s]$. This implies that $\Phi\left(B \oplus A_{j} ; b_{m}\right) \downarrow=\sigma[s]$ as required.

As usual we have

Lemma 6.7. If the hypothesis of $G_{\Phi, i, j}$ is correct then $\lim _{s \rightarrow \alpha} \ell(\Phi, i, j)[s]=\alpha$.

Proof. Let $\beta<\alpha$. We have $\Phi\left(B \oplus A_{i} ; \beta\right)=\Phi\left(B \oplus A_{j} ; \beta\right)$, and these computations hold from some stage $s$ onwards; after $s$ we always have $\ell(\Phi, i, j) \geqslant \beta$. 
At stage $s$, after some initial action by $P$, we determine which nodes are accessible by induction. If $\eta$ is a hole which is accessible, then $\eta^{\urcorner} 0$ is accessible; if $\rho$ is a gate and is expansionary at $s$, then $\rho^{\frown} 0$ is accessible, otherwise $\rho^{\frown} 1$ is. Note that the calculations of $\ell$ are done after $P$ 's action, and after some cascading took place. The definition of the accessible nodes ends when we get to a node which requires attention, or to a node that was just initialized (by $P$, or during cascading).

\section{The postal service comes to rescue on an eagle's wings}

Now here's a delicate point. We saw how to protect a follower's realization by finely managing its entourage's movements and cancelling weaker balls and markers. The idea is that at every stage from the follower's realization onward, all of the $\eta$-gates act in concert to protect $x$; we manage the entourage in such a way so that no $\eta$-gates would enumerate a troublesome marker, even if the computations making things believable grow and prosper.

There is a snag though, and it is the cascading. At any stage, to follow P's strategy, we dump many numbers into $B . x$ can prevent $B$ from directly injuring the computation realizing $x$ by imposing restraint on $P$. However, $P$ 's action may also make some $\Gamma_{\rho}$ computations (for $\rho$ an $\eta$-gate) unbelievable, prompting markers $\gamma_{\rho}$ to go into $B$, and perhaps destroy $x$ 's realization. If $\operatorname{dom} \Gamma_{\rho}(B)[s]$ is finite, then the total restraint that needs to be imposed on $P$ to prevent such a chain of events will be finite (i.e. bounded below $b_{\omega}[s]$ ). But at the beginning of some limit stages it could be the case that $\operatorname{dom} \Gamma_{\rho}(B)=\omega$, and then it is possible that enumerating any $b_{m}[s]$ into $B$ will prompt a cascading effect which will destroy $x$ 's realization. The amount of restraint needed to prevent this occurrence is infinite, and this is bothersome for $P$; in fact, this would derail the entire construction. 
The solution is to protect, for every follower $x$ and $\eta$-gate $\rho$, a computation $\Gamma_{\rho}\left\lceil n_{\rho}(x)\left(n_{\rho}\right.\right.$ is called a stamp $)$. The ultimate goal is that together, all stamps for $x$ set a barrier $\gamma$, making sure that for all $\eta$-gates $\rho, \Gamma_{\rho}\left\lceil n_{\rho}(x)\right.$ is believed due to some computation with use less than $\gamma$, but all the markers for computations $\Gamma_{\rho}\left(n_{\rho}(x)\right)$ are greater than $\gamma$. Thus all cascading must stop at $n_{\rho}(x)$.

The main idea in carrying out this strategy is that at every stage at which balls of $x$ 's entourage are enumerated into their sets, we activate the stamps $n_{\rho}(x)$, which simply means enumerating $\gamma_{\rho}\left(n_{\rho}(x)\right)$ into $B$, thereby removing unprotected $\Gamma_{\rho}$ computations. Thus at the end of the stage, we have, for all $\eta$-gates $\rho, \operatorname{dom} \Gamma_{\rho}(B)=$ $n_{\rho}(x)[s]$. That is, we ensure that there are no dangerous markers because all computations are protected.

This action, though, is not enough, and we now explain why. Suppose that at stage $s$, balls from $x$ 's entourage enter their sets, and that the new end of the entourage lies at gate $\rho^{*}\left(\right.$ so $\rho^{*}$ and all gates below it become safe at $\left.s\right)$. Now all $\Gamma_{\rho}\left\lceil n_{\rho}(x)\right.$ are only singly-believable, since it is most likely that computations for one side were injured by the aforesaid enumeration.

These computations come back slowly and gradually, from the lower $\eta$-gates up to $\rho^{*}$. But by the time we get to $\rho^{*}$ (i.e. at the first time $t$ it is expansionary since $s$ ), lower $\eta$-gates $\rho$ have defined computations $\Gamma_{\rho}\left(n_{\rho}(x)\right.$ ) (for they cannot wait for $\rho^{*}$ or for $x$ ), with uses $\gamma_{\rho}\left(n_{\rho}(x)\right)$ which are smaller than the use of the new $\Phi$ computation for $\rho^{*}$ (on the side that was injured). Now if we do nothing and activate all stamps at a later stage, we shoot ourselves in the foot, because we eliminate one side for $\rho^{*}$, while letting balls cross $\rho^{*}$ which will eventually destroy the other side, leaving us "bald on both sides". We violated the principle of protecting both sides when $\rho^{*}$ is safe, not by appointing small markers, but rather because of the necessary 
increase of the barrier $\gamma$. The solution is immediate: increase $n_{\rho}(x)$. We can do this because after $s$, all $\rho \subseteq \rho^{*}$ are safe. Note that for $\rho \supsetneq \rho^{*}$, we do not (and cannot) increase $n_{\rho}(x)$ since they are unsafe; but neither do we wish to, because they were not accessible between $s$ and $t$, and so didn't appoint new markers. Also note that such gates $\rho$ are not bothered by the entire pandemonium, since they are only protecting one side, which existed before $s$.

We can thus describe the complete instructions for handling the stamps. Let $\eta$ be a hole and $x$ be a follower. At stage $w_{0}, x$ is realized and released. At this stage we issue the stamps: we set $n_{\rho}(x)=\operatorname{dom} \Gamma_{\rho}(B)$ for all $\eta$-gates $\rho$. We noted earlier that this is finite.

Now at every stage $s$ at which balls from $x$ 's entourage get enumerated into their target sets, we activate all stamps (enumerate all $\gamma_{\rho}\left(n_{\rho}(x)\right)$ into $B$ for $\rho$ such that $\left.\Gamma_{\rho}\left(B, n_{\rho}(x)\right) \downarrow[s].\right)$

At each such stage $s$, we let $\rho^{*}=\rho^{*}(x, s)$ be the gate at which the new end of $x$ 's entourage is waiting. At the first stage $t>s$ at which $\rho^{*}$ is expansionary, we perform the echo effect: we redefine, for all $\rho \subseteq \rho^{*}, n_{\rho}(x)=\operatorname{dom} \Gamma_{\rho}(B)[t][\mathrm{We}$ note that $\Gamma_{\rho}(B)$ is doubly believable at $t$ because $\rho$ is expansionary at $\left.t\right]$. We also initialize all nodes weaker than $\eta$ and declare that $\rho^{*}$ is the last node accessible at $t$.

Note that $n_{\rho}(x)$ is only updated once after each stage at which $x$ enumerates balls, which we showed happens only finitely many times. Thus we have

Lemma 6.8. $n_{\rho}(x)$ is redefined finitely many times.

This implies that at limit stages, $n_{\rho}(x)$ is well-defined and finite. 


\section{Restraint}

Recall that the strategy to satisfy the $P$ requirement is at every stage $s$, to enumerate all but finitely many numbers $<\omega s$ into $B$. This clearly suffices. However, it is easily noticed that such wild action may be harmful to other requirements, and so, most nodes will wish to impose restraint on $P$. We need to make sure, though, that such restraint is indeed finite.

Thus at the beginning of the stage we initialize all nodes at levels greater than $n(s)$. Also, for every hole $\eta$, we eliminate all but the strongest $n(s)$ followers.

The restraint on $P$ at the beginning of $s$ consists of the following:

1. For every gate $\rho$, let $m_{\rho}[s]=\min \left\{n(s), \operatorname{dom} \Gamma_{\rho}(B)[s]-1\right\}$. Suppose that the computation $\Gamma_{\rho}\left(B, m_{\rho}[s]\right)$ was defined at some $t<s$. Then $\rho$ restrains $P$ from enumerating numbers below $\omega t$ into $B$.

2. For every hole $\eta$ and uncancelled follower $x$ for $\eta$ (even if already enumerated), $\eta$ restrains $P$ from enumerating numbers below $\omega t$, where $t<s$ is the last stage at which $x$ received attention.

3. $P$ restrains itself from enumerating $b_{n(s)}[s]$ into $B$.

The total restraint on $P$ is denoted by $r(s)$. To act, $P$ enumerates every number between $r(s)$ and $\omega s$ into $B$.

As defined, the restraint on $P$ imposed by any ball or node is finite (in the sense that it protects only a finite initial segment of $\neg B$ from entering $B$. The instructions will show that at the beginning of every stage, after removing balls and initializing nodes, there are only finitely many balls on the machine and finitely many noninitialized nodes. We thus have: 
Lemma 6.9. For every $s$, if $\neg B \cap \omega s$ is infinite, then $r(s)<\omega s$.

\section{Attention}

Suppose that $\eta$ is a hole which is accessible at stage $s$. We say that $\eta$ requires attention if it is not yet satisfied, and if one of the following holds:

1. $\eta$ has a follower $x$ on the machine, and $x$ 's entourage's end is waiting at a gate.

2. $\eta$ has a follower $x$ waiting at the hole, and $x$ is realized.

3. There is no follower waiting at the hole.

We also say that a gate $\rho$ requires attention if

4. $\rho$ is an $\eta$-gate for some hole $\eta$, which has a follower $x$ on the machine; at some stage $t<s$, numbers from $x$ 's entourage were enumerated into their sets, and $s$ is the least stage after $t$ at which $\rho$ is expansionary, and the new end of $x$ 's entourage is now lying at $\rho$.

In cases (1) and (2) we say that $x$ requires attention, and we give attention to the strongest such $x$ (while removing all weaker followers). In case (1), we let the permissible tail of the entourage's end move to the next $\eta$-gate, or to the permitting bin if there is no such gate. In case (2), We let $x$ 's entire entourage move to the highest $\eta$-gate; we issue stamps.

In case (3), we appoint a large new follower $x$ and place it at the hole. If $\rho$ requires attention (case (4)), we perform the echo effect for $x$ : we redefine stamps as described above, and initialize nodes weaker than $\eta$. 


\section{Enumeration}

After P's action, and before defining accessible nodes, we check to see if some balls in the permitting bin are permitted. If there are some such, we choose the strongest follower $x$ (from the strongest hole $\eta$ ) which has numbers permitted; we initialize nodes weaker than $\eta$, remove followers for $\eta$ which are weaker than $x$, enumerate the numbers into their sets, and activate all stamps $n_{\rho}(x)$.

If a follower $x$ was enumerated, then all other followers for its hole are cancelled, and the hole declares victory and considers itself satisfied (until the next stage at which it is initialized) [If $x$ is ever cancelled, it is because the hole is initialized]. We will see that indeed if $\eta$ declares victory and is not later initialized then its requirement is met.

\section{Defining $\Gamma_{\rho}$}

Suppose that $\rho$ is expansionary at $s$. Then when we get to $\rho$, we wish to extend $\Gamma_{\rho}$. If $m=\operatorname{dom} \Gamma_{\rho}(B)[s]$ is under agreement (which means that $\Phi\left(B \oplus A_{i} ; b_{m}\right) \downarrow=$ $\left.\Phi\left(B \oplus A_{j} ; b_{m}\right) \downarrow[s]\right)$, then we define $\Gamma_{\rho}(B, m)$ with the correct value and with a new, large marker.

\subsubsection{Construction}

We give the instructions for stage $s$ of the construction.

- Act for $P$ : initialize all nodes of length $\geqslant n(s)$. Also, remove all followers for holes $\eta$, except for the strongest $n(s)$ many followers. Calculate the restraint on $P$, and let $P$ dump balls into $B$. Winnow entourages, to make them finite: remove all balls (in all entourages), which are at place $k$ in their entourage, 
where $b_{k}[s]>r(s)$. [Note that a follower is never winnowed]. Now remove unbelievable $\Gamma_{\rho}$ computations and cascade.

- See if some balls in the permitting bin are permitted. If so, enumerate balls, initialize as described, and skip the next sub-stage. Remove unbelievable $\Gamma_{\rho}$ computations and cascade.

- Find which nodes are accessible and require attention; extend $\Gamma_{\rho}$ functionals for $\rho$ which are expansionary (where it is possible) and then act for the first node which requires attention. Perform the echo effect if necessary.

- Appoint new traces at the end of every entourage.

That's the construction.

\subsubsection{Verifications}

\section{Success of $P$}

At every stage, the restraint on $P$ is finite, which implies that otp $\neg B \cap s<\omega$ for all $s<\alpha$; thus otp $\neg B \leqslant \omega$.

Lemma 6.10. otp $\neg B \geqslant \omega$.

Proof. By induction on $k$, we show that $|\neg B| \geqslant k$. Suppose this holds for $k$. There is some stage $s_{k}>\beta_{k+1}$ such that for $i<k, b_{i}\left[s_{k}\right]=b_{i}$. Consider the stage $t_{k}>s_{k}$ at which $b_{k}\left[s_{k}\right]$ enters $B$ (if there is no such stage then we're done). After $P$ 's action we have $b_{k}\left[t_{k}\right]=\omega t_{k}$; this number is never chosen as a marker.

After stage $t_{k}>\beta_{k+1}, P$ is restrained from enumerating $b_{k}\left[t_{k}\right]$ into $B$. Thus $b_{k}\left[t_{k}\right]=b_{k}$ and $|\neg B| \geqslant k+1$. 
We also note that the $A_{i}$ are amenable; at stage $s$, after $P$ 's action, we have only finitely many balls on the machine. New balls are appointed large. Hence $A_{i}\left\lceil s\right.$ differs from $A_{i}[s] \uparrow s$ by only finitely many numbers, hence is $\alpha$-finite.

The same observation gives us even more.

Lemma 6.11. For all $X$, if $A_{i} \leqslant w \alpha$ then $A_{i} \leqslant \alpha X$.

Proof. To enumerate $A_{i} \uparrow s, X$ can first look at the construction at stage $s$. A look on the machine reveals finitely many balls which have a chance to enter $A_{i}$; by assumption, $X$ can tell which ones will, and so determine $A_{i}\lceil s$.

\section{The concert is sonorous and harmonious}

Lemma 6.12. Suppose that $x$ is a follower for $\eta$ which works for $H_{\Psi, k}$, and that $x$ is realized at some stage and then dropped from its hole. Then as long as $x$ is not cancelled (even after it is enumerated), it is still realized by the original computation.

Proof. Suppose that $x$ is dropped from the hole at $t_{0}$. Let $s_{1}, s_{2}, \ldots, s_{N}$ be the stages at which members of $x$ 's entourage are enumerated into their target sets, and let $t_{i}$ be the stage at which the echo effect (of $s_{i}$ ) occurs. We let, for $i<N$, $\rho_{i}^{*}$ be the $\eta$-gate at which the end of $x$ 's entourage lies at the beginning of $t_{i}$ (so $\rho_{0}^{*}$ is the highest $\eta$-gate).

For every $\eta$-gate $\rho$ (working for $G_{\Phi, i, j}$ ) and stage $s$ at which there are numbers of $x$ 's entourage below $\rho$, there is one side $l_{\rho}(x)[s] \in\{i, j\}$ which is protected from injury: no balls targeted for $l_{\rho}(x)[s]$ crossed $\rho$ at the last time balls from $x$ 's entourage crossed $\rho$.

At stage $s$, the computation $\Phi\left(B \oplus A_{l} ; n_{\rho}(x)\right)[s]$ is protected for $l=l_{\rho}(x)[s]$ or for both $l \in\{i, j\}$ if $s \in\left(t_{i}, s_{i+1}\right]$ and $\rho \subseteq \rho^{*}$. 
A quick inspection shows that the only danger to protected computations may come from markers for $\eta$-gates. For by definition, balls from $x$ 's entourage cannot destroy a protected computation; the moment that balls from $x$ 's entourage, targeted for $A_{i}$, cross an $\eta$-gate $\rho$, The computation $\Phi\left(B \oplus A_{i} ; n_{\rho}(x)\right)$ ceases to be protected. Of course stronger balls don't move before $x$ is cancelled; the same goes for marker for gates $\rho$ such that $\rho^{\frown} 0<_{L} \eta$. Balls weaker than $x$ and markers weaker than $\eta$ are always cancelled when $x$ receives attention, in particular in every $t_{i}$ and $s_{i}$, so are large. Also note that $\eta$ imposes sufficient restraint on $P$ to prevent this dumping requirement from intervening.

By induction on $s \geqslant t_{0}$ we can show that all protected computations are preserved (from the moment they are declared protected until they cease to be protected) and that all markers $\gamma_{\rho}\left(n_{\rho}(x)\right)[s]$, if defined, are large. This is because after each $t_{i}$ and $s_{i}$, for all $\eta$-gates $\rho$, we have $\Gamma_{\rho}\left(B, n_{\rho}(x)\right) \uparrow[s]$, so new markers are chosen large. Thus by induction we can show that if $\Phi\left(B \oplus A_{l} ; n_{\rho}(x)\right)[s]$ is protected, then $\Gamma_{\rho}\left(B ; n_{\rho}(x)\right)[s]$ is $l$-believable and so small markers are not enumerated into $B$.

\section{Fairness, true path and success of the holes.}

Lemma 6.13. If $\eta$ is a hole which is accessible $\alpha$ many times and eventually never initialized, then eventually $\eta$ stops acting.

Proof. If there is a follower which is never realized or cancelled, then after appointing it, $\eta$ never acts (and wins). If after $r^{*}=\operatorname{init}(\eta)[\alpha]$, a follower for $\eta$ is enumerated into its target set (say at stage $s>r^{*}$ ), then at $s$, all other followers are removed and realization of the follower is preserved; after $s, \eta$ does not act, and it wins. 
Assume that no follower is ever enumerated into the target set after $r^{*}$ and that every appointed follower is later either cancelled or realized (in which case some entourage part gets stuck in the permitting bin).

Let $W$ be an r.e. set defined as follows: $s>r^{*}$ is enumerated into $W^{[m]}$ if an $m^{\text {th }}$ follower for $\eta$ at $s$ receives attention at $s$.

For all $t>\beta_{m}$ we have $n(t)>m$. Thus after $\beta_{m}$, no $k^{t h}$ follower for $\eta$, for $k \leqslant m$, can be removed by $P$. Every follower receives attention finitely many times; it follows (by induction on $k \leqslant m$ ) that there are only finitely many $k^{\text {th }}$ followers appointed after stage $\max \left\{\beta_{m}, r^{*}\right\}$. It follows that $W^{[m]} \backslash \beta_{m}$ is finite. By the properties of $f$ and $g$, there is some large $m$ such that $g(m)>\max W^{[m]}$.

Let $x$ be the last $m^{\text {th }}$ follower appointed. We claim that $x$ cannot have balls stuck indefinitely in the bin. For suppose there are, and that they got to the bin at stage $s . s$ enters $W^{[m]}$, and so $g(m)>s$. Since $g(m)[s]<s$, we must have a change in $g(m)$ at some stage $t>s$, at which $x$ is permitted and the balls would be enumerated into their sets; contradiction.

We define the true path, as usual, to be the left-most nodes which are visited $\alpha$ many times. We can now prove fairness.

Lemma 6.14. A node on the true path is not injured $\alpha$ many times.

Proof. By induction on the length of the node. Suppose this holds for all $\tau \subsetneq \sigma$ where $\sigma$ is on the true path. We know that eventually every $\tau \subsetneq \sigma$ stops initializing $\sigma$.

Of course, after some stage $P$ does not injure $\sigma$.

Let $s_{0}$ be a stage after which neither $P$ nor any $\tau \subsetneq \sigma$ injure $\sigma$, and after which no node to the left of $\sigma$ is accessible. At $s_{0}$, there are finitely many nodes to the 
left of $\sigma$ which are not initialized at $s_{0}$.

Holes $\eta<_{L} \sigma$ have finitely many followers at $s_{0}$, and do not appoint any new ones. Each such follower initializes $\sigma$ at most twice (when elements of its entourage are enumerated into their sets, and later when an echo effect is performed for that enumeration).

Gates $\rho$ such that $\rho^{\frown 0}<_{L} \sigma$ have finitely many $\Gamma_{\rho}$ computations defined at $s_{0}$, and never define more. $\rho$ may injure $\sigma$ each time it removes computations, but this occurs finitely many times.

Thus eventually no node to the left of $\sigma$ injures $\sigma$.

\section{$D$ knows all}

Lemma 6.15. $A_{i} \leqslant \alpha$.

Proof. Say that $x<\alpha$, and that $D$ is asked whether $x \in A_{i}$ or not. First, $D$ takes a look at stage $x$; if $x$ is not a ball on the machine, targeted for $A_{i}$, and is not yet in $A_{i}$, then it will not enter $A_{i}$.

Suppose that $x$ is a ball, which is part of the $m^{\text {th }}$ entourage for some hole $\eta$.

$D$ can calculate a stage $t$ after which $m$ will never be permitted. After this stage, $x$ will not be permitted by $D$. Thus if $x$ is not yet in $A_{i}$ at $t$ and is still on the machine, $D$ can guarantee that $x$ will not be enumerated into $A_{i}$ : it will either be cancelled, or parts of its entourage will for every lie in the permitting bin.

We now need to see how $D$ can compute $B$. P's action is not a problem here, as it is restrained by $n(s)$, and $\left\langle\beta_{k}\right\rangle$ is computable from $D$. Also, since $D$ knows $A_{i}$, it can predict which balls will activate stamps. However, $D$ cannot predict what kind of effect cascading may have on $B$. To overcome this problem we note that (as we needed for the success of the stamps), there are naturally occurring 
"dams" against cascading which protect $B$ and can be detected by $D$. We define some $\gamma$ to be stable at $s>\gamma$ if the following hold:

1. For all $\rho$ (working for $G_{\Phi, i, j}$ ), if $\Gamma_{\rho}(B, m) \downarrow[s]$ and $\gamma_{\rho}(m)<\gamma$, then there is an $l \in\{i, j\}$ such that $\Gamma_{\rho}(B, m)$ is $l$-believable at $s$ by a $\Phi$-computation with use less than $\gamma$.

2. If $x$ is an $m^{\text {th }}$ follower for its hole at $s$ and there is a stamp $n_{\rho}(x)[s]$ such that $\gamma_{\rho}\left(n_{\rho}(x)\right)[s]<\gamma$, then $m$ will never be permitted after $s$.

3. If $b_{k}[s]<\gamma$ then $s>\beta_{k}$.

4. For $i<3, A_{i} \uparrow \gamma[s]=A_{i} \uparrow \gamma$.

Lemma 6.16. If $\gamma$ is stable at $s$ then $B\lceil\gamma[s]=B \uparrow \gamma$.

Proof. The third condition implies that after $s, P$ will not enumerate numbers smaller than $\gamma$ into $B$.

The second condition guarantees that no activations of stamps put numbers smaller than $\gamma$ into $B$ after $s$.

We show by induction on $t \geqslant s$ that no numbers smaller than $\gamma$ enter $B$ at stage $t$. Induction up to $t$, together with the fourth condition, imply that at $t$, all $\Gamma_{\rho}$ computations with small markers (which necessarily were defined prior to $s$ ) are believable via the same computations which existed at stage $s$ (by the first condition). It follows that at $t$, no such markers are put into $B$ by gates removing unbelievable computations.

Lemma 6.17. There are unboundedly many $\gamma$ such that for some $t>\gamma, \gamma$ is stable at $t$. 
Proof. Suppose that at some stage $w$, a follower $x^{*}$ for a hole $\eta^{*}$ which lies on the true path is enumerated into its target set, and further suppose that $\eta^{*}$ (and so $\left.x^{*}\right)$ is never injured after $w$. Let $\gamma=\omega w$.

Any $y<x^{*}$ which is on the machine at $w$ is stronger than $x^{*}$, hence will never receive attention. All weaker balls are cancelled at $w$, and new ones appointed are large. It follows that for $i<3, A_{i} \uparrow \gamma[w]=A_{i} \uparrow \gamma$.

At $w$, all stamps $n_{\rho}\left(x^{*}\right)$ for $\eta^{*}$-gates $\rho$ are activated. As was shown in the proof of lemma 6.12, The computations $\Gamma_{\rho} \uparrow n_{\rho}\left(x^{*}\right)$ are permanent, so markers enumerated by $\eta^{*}$-gates after $s$ are picked after $w$ hence are greater than $\gamma$. No other gates may enumerate markers which are smaller than $\gamma$, and $P$ too is prevented (by $x^{*}$ ) from enumerating numbers smaller than $\gamma$ into $B$. It follows that $B \uparrow \gamma[w]=B \uparrow \gamma$

Suppose that $s>w$ and suppose that $\rho$ is a gate and that $\Gamma_{\rho}(B, m) \downarrow[s]$ with use $\gamma_{\rho}(m)[s]<\gamma$. Necessarily, this computation was defined before $w$ and was believable at $w$, by a $\Phi$-computation with use $<\gamma$ (at $w$, all computations have use $<\gamma)$. This $\Phi$-computation is preserved.

We can thus let $s$ be a stage greater than $w$ and $\beta_{k}$ (where $\left.\gamma=b_{k}[w]\right)$ and late enough such that $n(w)$ will not be permitted after $s$. We claim that $\gamma$ is stable at s. This suffices since we can clearly find such $\gamma$ as large as we like.

We know that the first, third and fourth conditions hold. We show that the second condition holds too. Suppose that at $s, x$ is a follower on the machine and there is a stamp $n_{\rho}(x)[s]$ such that $\gamma_{\rho}\left(n_{\rho}(x)\right)[s]<\gamma$. Necessarily, the computation $\Gamma_{\rho}\left(B, n_{\rho}(x)[s]\right)$ is defined prior to stage $w$, and does not change until $s$. Every follower $y$ appointed at $t>w$ which defines a stamp $n_{\rho}(y)$ will have $n_{\rho}(y)=$ $\operatorname{dom} \Gamma_{\rho}(B)[t]>n_{\rho}(x)[s]$. Hence $x$ is already on the machine at $w$, so is the $m^{t h}$ 
follower for its hole, $m<n(w)$ (other followers are removed at $w$ ). After $s, x$ will not be permitted.

Finally, we can see that $D$ can tell whether some $\gamma$ is stable at a stage $s$. The first condition is computable. The second can be decided by $D$ because it controls the permitting. The third, because $D$ can compute $\beta_{n}$; and the last, because $A_{i} \leqslant \alpha$. We have proven

Lemma 6.18. $B \leqslant_{\alpha} D$.

\section{The Top}

Lemma 6.19. $A_{i} \leqslant \alpha B \oplus A_{j} \oplus A_{k}$.

Proof. To find if $x \in A_{i}$, we first look at stage $x$, to see if $x$ is a ball on the machine, targeted for $A_{i}$. If so, at that stage, $x$ is the $n^{\text {th }}$ element of its entourage. Using $B$ we can find a stage $s>x$ such that $b_{n+1}[s]=b_{n+1}$. If $x$ is still on the machine at $s$, then a trace for $x$ will never be winnowed after $x$.

The usual argument shows that after $s, x$ has only finitely many traces, and the last one either enters its set at the same time $x$ enters $A_{i}$, or doesn't, in which case $x \notin A_{i}$.

\section{Success of the Gates}

Lemma 6.20. Suppose that $\rho$ working for $G_{\Phi, i, j}$ is on the true path, and assume that the hypothesis of $G_{\Phi, i, j}$ holds. Then $\Gamma_{\rho}(B)=\Phi\left(B \oplus A_{i}\right)$.

Proof. $\rho$ is expansionary $\alpha$ many times.

By induction on $n$, we show that $\Gamma_{\rho}(B, n) \downarrow=\Phi\left(B \oplus A_{i} ; b_{n}\right)$. Assume up to $n-1$ and assume that after $s_{0}, \rho$ is never initialized; for $m<n, \Gamma_{\rho}(B, m)$ computations 
are defined with correct markers (so they will not end up in $B$ ) and correct $\Phi$ computations from both sides; $b_{n}\left[s_{0}\right]=b_{n}$, and $\Phi\left(B \oplus A_{l} ; b_{n}\right)$ is correct for both $l \in\{i, j\}$. Assume also that $s_{0}$ is expansionary.

At every expansionary stage $s \geqslant s_{0}, \Gamma_{\rho}(B ; n)$, if not already defined, gets defined (because the agreement between $\Phi\left(B \oplus A_{i}\right)$ and $\Phi\left(B \oplus A_{j}\right)$ is permanent). At $s_{0}$, if we have an incorrect computation, then it gets removed; all later computations are always doubly believable (and give the correct answer). Such a computation cannot be removed by $P$ (it is prohibited; $n(s)>n$ ). The believability also implies that such a computation cannot be cancelled during cascading. The only reason that $\Gamma_{\rho}(n)$ may be cancelled again is that $n=n_{\rho}(x)$ for some follower $x$.

At $s_{0}$, there are finitely many $x$ s on the machine such that $n_{\rho}(x)=n$, thus eventually (after some $s_{1}$ ), none of these balls activate their stamps.

If at $s \geqslant s_{0}$, some follower $y$ defines $n_{\rho}(y)$, then $\rho$ is expansionary at $s$. Thus before the definition, $\rho$ got to define $\Gamma_{\rho}(n)$. Thus $n_{\rho}(y)>n$. Thus after $s_{1}$, no stamp $n_{\rho}(x)=n$ will be activated.

Thus we eventually get a permanent computation. 


\section{APPENDIX A}

\section{BASICS}

It is convenient to use Jensen's $J_{\alpha}$ hierarchy. This is a cumulative hierarchy of the constructible universe $L$. Readers who are unfamiliar with this hierarchy, may ignore the letter $J$ throughout this work and replace it by the letter $L$. A formal definition of the $J_{\alpha}$ hierarchy and an investigation of its basic properties can be found in texts such as Jech [Jec03] or Dodd [Dod82]. The advantage of using $J_{\alpha}$ over $L_{\alpha}$ is that it behaves nicely with respect to the notion of the projectum, which is discussed in appendix C. Essentially, $J_{\alpha+1}$ is obtained from $J_{\alpha}$ by closing $J_{\alpha} \cup\left\{J_{\alpha}\right\}$ under a finite collection of rudimentary functions (similar to the Gödel operations). $J_{\alpha} \cap \mathrm{On}=\omega \alpha$, the $\alpha^{t h}$ limit ordinal.

For our purposes, let an amenable structure be a structure of the form $\left(J_{\beta}, \in, X\right)$ where $\beta \geqslant 1$ is an ordinal and $X \subset J_{\beta}$ is amenable (set) for $J_{\beta}$, that is, for every $x \in J_{\beta}, X \cap x \in J_{\beta}$. Amenable sets are also called regular.

Since $\in$ is a relation in all amenable structures, we suppress it in notation and refer to the structure $\left(J_{\beta}, X\right)$. We write $J_{\beta}$ for both the set and the structure $\left(J_{\beta}, 0\right)$. If $M=\left(J_{\beta}, X\right)$ is an amenable structure then we sometimes use $M$ to denote the universe $J_{\beta}$.

The Levy hierarchy for formulas in the language of set theory, augmented by a unary predicate, is the familiar one. For any amenable structure $M, \Delta_{0}(M)$ denotes the collection of subsets of $M$ defined by bounded formulas, with parameters in $M$. Similarly we have $\Sigma_{n}(M), \Pi_{n}(M)$.

Fact. If $M$ is an amenable structure then every $A \in \Delta_{0}(M)$ is amenable for $M$.

Various set-theoretic notions have also recursion-theoretic names. Let $M$ be an amenable structure. A set $x$ is $M$-finite if $x \in M$ (we usually say $\beta$-finite 
instead of $J_{\beta}$-finite). The sets which are $\Sigma_{1}(M)$ are also called $M$-recursively enumerable. $\Delta_{1}(M)$ sets (i.e. those which are both $M$-r.e. and $M$-co-r.e.) are called $M$-recursive. A partial function $f: M \rightarrow M$ is $M$-recursive if it is $M$-r.e. (as a set of pairs).

Fact. For every $\beta$, there is a $\beta$-recursive bijection between $J_{\beta}$ and $\omega \beta=J_{\beta} \cap$ On.

Thus, for the purposes of recursion theory, we do not care if the sets considered are subsets of $J_{\beta}$ or of $\omega \beta$.

We write $\beta$-r.e. instead of $J_{\beta}$-r.e. and $\beta$-recursive instead of $J_{\beta}$-recursive. When $\beta$ is fixed we omit the prefix $\beta$.

We never write "finite" instead of $\beta$-finite. It is in fact the theme of this thesis to exhibit the fundamental difference between finite and $\beta$-finite.

An amenable structure $M$ is admissible if the image of every $M$-finite set, under an $M$-recursive function, is bounded (say in $<_{J}$ ). An amenable set $X \subset J_{\beta}$ is called admissible (when $\beta$ is fixed) if $\left(J_{\beta}, X\right)$ is admissible. Admissible sets are also called "regular and hyperregular". An ordinal $\alpha$ is admissible if $J_{\alpha}$ is an admissible structure. Throughout this thesis, $\alpha$ always denotes an admissible ordinal.

Fact. Let $M=\left(J_{\alpha}, X\right)$ be admissible.

1. $\alpha=\omega \alpha ; J_{\alpha}=L_{\alpha}$.

2. $M$ satisfies $\Delta_{1}$-comprehension: every $A \in \Delta_{1}(M)$ is amenable for $M$.

3. $M$ satisfies $\Sigma_{1}$-bounding: the image of an $M$-finite set under an $M$-recursive function is $M$-finite. In fact, if $f$ is partial $M$-recursive, $x$ is $M$-finite and $x \subset \operatorname{dom} f$, then $f \uparrow x$ is $M$-finite. 
4. If $A$ is $M$-r.e. then $\forall x \in y A$ is also $M$-r.e.

5. All familiar elementary theorems of $\omega$-recursive function theory hold (enumeration theorem, s-m-n theorem, definition by induction, recursion theorem, etc.)

A semi-admissible structure is an amenable structure $\left(J_{\alpha}, X\right)$ where $\alpha$ is admissible. We will almost always work with semi-admissible structures.

\section{Functionals and Reducibilities}

Let $M=\left(J_{\beta}, X\right)$ be an amenable structure. An $M$-enumeration functional is an $M$-r.e. set whose elements are pairs $(p, x)$, where $p$ is a partial string, that is, a function with domain a set of ordinals and range 2 .

If $\Psi$ is an $M$-enumeration functional and $p$ is any partial string (not necessarily $M$-finite), we let

$$
\Psi(p)=\left\{x: \exists p_{0} \subset p\left[\left(p_{0}, x\right) \in \Psi\right]\right\}
$$

Let $A \subset \omega \beta$. We say that $B \subset M$ is $M$-r.e. in $A$ if there is some $M$-enumeration functional $\Psi$ such that $B=\Psi(A)$. A partial function is $M$-weakly recursive in $A$ if it is $M$-r.e. in $A$ as a set of pairs. A set $B \subset \alpha$ is $M$-weakly recursive in $A$ (we write $\left.B \leqslant_{w M} A\right)$ if it is both $M$-r.e. and $M$-co-r.e. in $A$.

An $M$-functional (also known as a Turing functional or as a strong functional) is an $M$-enumeration functional whose range also consists of partial strings. When we use an $M$-functional $\Psi$ we use $\cup \Psi(p)$ instead of $\Psi(p)$ (and write $\Psi(p)$ for the former, just to confuse things. However we only use this notation if $\Psi(p)$ is a function.) 
Let $A, B \subset \omega \beta . B$ is strongly $M$-r.e. (or tamely $M$-r.e.) in $A$ if

$$
\{K \subset B: K \in M\}
$$

is $M$-r.e. in $A$ (Here we regard $B$ as a set rather than as its characteristic function).

$B$ is $M$-recursive in $A$ (we write $B \leqslant_{M} A$ ) if both $B$ and $\neg B=\omega \beta \backslash B$ are strongly $M$-r.e. in $A$. $B$ is $M$-recursive in $A$ iff

$$
\{p \in M: p \text { is a partial string \& } p \subset B\}
$$

is $M$-r.e. in $A$.

The relation $\leqslant_{M}$ is a reflexive and transitive relation on the subsets of $\alpha$. The equivalence classes are called $M$-degrees, or $X$-degrees (when $\alpha$ is understood). $J_{\alpha}$-degrees are usually called $\alpha$-degrees.

Lemma A.1. Let $M=\left(J_{\beta}, X\right)$ be amenable and let $A \subset \omega \beta$ be amenable. Let $B \subset M$. Then $B$ is $M$-r.e. in $A$ iff it is $(M, A)$-r.e.

Proof. Suppose that $B=\Phi(A)$ where $\Phi$ is an $M$-enumeration functional. Then $x \in B \leftrightarrow \exists p \subset A[(p, x) \in \Phi]$ which is $\Sigma_{1}(M, A)$.

Suppose that $B \in \Sigma_{1}(M, A)$ (defined by the formula $B(x)$; say $B$ is over $S_{\gamma}$, $\gamma<\omega \beta$.) Let

$$
\Phi=\left\{(p, x): \operatorname{dom} p>\gamma \&\left(S_{\operatorname{dom} p}, X\lceil\operatorname{dom} p, A\lceil\operatorname{dom} p) \models B(x)\}\right.\right.
$$

Then $\Phi(A)=B$.

Thus $B \leqslant_{w M} A$ iff $B$ is $(M, A)$-recursive.

Lemma A.2. Let $M=\left(J_{\beta}, X\right)$ be amenable and let $A \subset \omega \beta$ be amenable. For all $A, B, C \subset \omega \beta, B$ is $M$-r.e. in $A \oplus C$ iff it is $(M, A)$-r.e. in $C$. 
Note that this follows from lemma A.1 if $C$ is amenable.

Proof. Suppose that $\Phi$ is an $M$-enumeration functional such that $A=\Phi(B \oplus C)$. Let

$$
\Psi=\{(p, x): \exists q \subset A[((q, p), x) \in \Phi]\}
$$

$\Psi$ is $(M, A)$-r.e. and $\Psi(C)=B$.

Suppose that $\Psi$ is $(M, A)$-r.e. and that $\Psi(C)=B$. Then by lemma A.1, $\Psi$ is $M$-r.e. in $A$; say $\Theta$ is $M$-r.e. and $\Theta(A)=\Psi$. Let

$$
\Phi=\{((q, p), x) \in M:(q,(p, x)) \in \Theta\}
$$

Then $\Phi$ is $M$-r.e. and $\Phi(A \oplus C)=B$.

Corollary A.3. Let $M=\left(J_{\alpha}, X\right)$ be amenable and let $A \subset \alpha$ be amenable. For all $B, C \subset \alpha, B \leqslant_{M} A \oplus C$ iff $B \leqslant_{M, A} C$ and $B \leqslant_{w M} A \oplus C$ iff $B \leqslant_{w(M, A)} C$.

Thus the $(M, A)$-degrees are isomorphic to the cone of $M$-degrees above $A$.

Lemma A.4. If $M$ is admissible, then every $M$-r.e. set is also strongly $M$-r.e.

Thus if $\left(J_{\alpha}, A\right)$ is admissible then for all $B, B \leqslant_{w \alpha} A$ iff $B \leqslant_{\alpha} A$.

\section{Regular Functionals}

From now, we fix an admissible ordinal $\alpha$ and work, for simplicity, in $J_{\alpha}$. All results can be relativized to admissible structures $\left(J_{\alpha}, X\right)$.

An enumeration functional $\Psi$ is called regular if its domain consists of strings - partial strings whose domain is an ordinal.

Lemma A.5. Let $\Psi$ be an enumeration functional. There is an regular enumeration functional $\Phi$ such that for all amenable $A \subset \alpha, \Phi(A)=\Psi(A)$. 
An r.e. index for $\Phi$ can be uniformly obtained from an r.e. index for $\Psi$.

Proof. Let $\mathcal{S}$ be the set of $\alpha$-finite strings, and let

$$
\Phi=\{(p, x): p \in \mathcal{S} \& \quad \& \in \Psi(p)\}
$$

Let $p$ be a string. $\Phi(p) \subset \Psi(p)$. On the other hand, if $p_{0}$ is a partial string, $x \in$ $\Psi\left(p_{0}\right)$ and $p$ is any string extending $p_{0}$ then $x \in \Phi(p)$. The conclusion follows.

Claim A.6. Let $A, B \subset \alpha$ and suppose that $B$ is amenable. Then $B \leqslant_{\alpha} A$ iff

$$
\{B \uparrow \beta: \beta<\alpha\}
$$

is r.e. in $A$.

Proof. Easy, because given a string $p$ we can (uniformly) enumerate all partial strings extended by $p$.

A functional is regular if it is regular as an enumeration functional and if its range consists of strings.

Lemma A.7. Let $A, B \subset \alpha$ be amenable. Then $B \leqslant_{\alpha} A$ iff there is some regular functional $\Phi$ such that $B=\Phi(A)$.

An index for $\Phi$ and an index for a reduction of $B$ to $A$ are uniformly interchangeable.

Proof. If $\Psi$ is a regular enumeration functional which enumerates, with oracle $A$, all $\alpha$-finite partial strings extended by $B$, then simply take $\Phi=\Psi \cap \mathcal{S}^{2}$, where as before $\mathcal{S}$ is the set of $\alpha$-finite strings. On the other hand, if $\Phi$ is a regular functional and $\Phi(A)=B$ then considered as an enumeration functional, $\Phi(A)$ enumerates $B \uparrow \beta$ for cofinally many $\beta$ s; one can now enumerate all strings extended by strings in $\Phi(A)$. 


\section{Recursively Enumerating Recursively Enumerable Sets}

Suppose that $A$ is an r.e. set. $A$ is identified with the formula defining it over $J_{\alpha}$. Assume the parameters used lie in $J_{\beta}(\beta<\alpha)$. $\beta$ can be uniformly obtained from an index for $A$ (which can be taken to be the formula $A$. In this way we identify the set $A$ with its index).

We let $A[s]=0$ for $s<\beta$ and for $s \geqslant \beta$ we let

$$
A[s]=\left\{x \in J_{s}: J_{s} \models A(x)\right\} .
$$

The sequence $\langle A[s]\rangle_{s<\alpha}$ is increasing, continuous, recursive and

$$
\bigcup_{s<\alpha} A[s]=A
$$

\section{Nice Functionals}

If $\Phi$ is an enumeration functional then for all $s, \Phi[s]$ is an enumeration functional too. We write $\Phi(p)[s]$ for $\Phi[s](p)$. The relation $x \in \Phi(p)[s]$ is recursive.

Definition. A regular enumeration functional $\Phi$ is nice if for all $\alpha$-finite $p$ and $x$, $(p, x) \in \Phi$ iff $x \in \Phi(p)[\operatorname{dom} p]$.

If $\Phi$ is a nice enumeration functional then for all $\alpha$-finite $p$ and $x, x \in \Phi(p)$ iff $(p, x) \in \Phi$; this is because $\Phi[s]$ is increasing. If $\Phi$ is nice then $\Phi$, and so the relation $x \in \Phi(p)$, are recursive.

Lemma A.8. Let $\Psi$ be a regular enumeration functional. There is some nice enumeration functional $\Phi$ such that for all amenable $A \subset \alpha, \Phi(A)=\Psi(A)$.

A recursive index for $\Phi$ can be uniformly obtained from an r.e. index for $\Psi$.

Proof. Let

$$
\Phi=\{(p, x): x \in \Psi(p)[\operatorname{dom} p]\}
$$


Note that if $\Phi$ is nice then the map $p \rightarrow \Phi(p)$ (ranging over $\alpha$-finite strings $p$ ) takes values in $J_{\alpha}$ and is recursive.

Definition. A functional $\Phi$ is nice if it is nice, considered as an enumeration functional, and furthermore:

1. It is consistent: for all strings $p, \Phi(p)$ is a string.

2. It is downward closed: For all strings $p$ and $q$, if $q \subset \Phi(p)$ then $(p, q) \in \Phi$.

Lemma A.9. Let $\Psi$ be a functional. There is some nice functional $\Phi$ such that for all amenable $A$, if $\Psi(A)$ is a function then $\Phi(A)=\Psi(A)$.

Proof. We may assume that $\Psi$ is a nice enumeration functional. Now let

$$
\Phi=\{(p, q): \Phi(p) \text { is a function \& } q \subset \Phi(p)\}
$$

Note that if $\gamma<\alpha, q \in J_{\gamma}$ and $q_{0} \subset q$ is a string then $q_{0} \in J_{\gamma}$. This preserves the fact that $\Phi$ is nice as an enumeration functional.

As was discussed before, we have:

Corollary A.10. Let $A, B \subset \alpha$ be amenable. Then $B \leqslant_{\alpha} A$ iff there is some nice functional $\Phi$ such that $\Phi(A)=B$.

Sometimes an enumeration functional is used to enumerate graphs of functions. We then call it a weak Turing functional. Such a functional $\Phi$ is called nice if it is nice as an enumeration functional and it satisfies consistency: for all strings $p$, $\Phi(p)$ is a function (but not necessarily with ordinal domain). Similarly to lemma A.9, we get 
Lemma A.11. Let $\Psi$ be an enumeration functional. There is some nice weak functional $\Phi$ such that for all amenable $A$, if $\Phi(A)$ is a function then $\Phi(A)=\Psi(A)$. Proof. Again we may assume that $\Psi$ is a nice enumeration functional, and we let

$$
\Phi=\{(p, x): x \in \Phi(p) \& \Phi(p) \text { is a function }\}
$$

Thus for amenable $A \subset a$ and any $B \subset \alpha, B \leqslant{ }_{w \alpha} A$ iff $\Phi(A)=B$ for some nice weak functional $\Phi$.

\section{Use}

If $\Phi$ is a nice functional, $p$ is a string and $\beta \leqslant \operatorname{dom} \Phi(p)$, then we let $\Phi(p ; \beta)=$ $\Phi(p) \uparrow \beta$ and $\phi(p ; \beta)$ be the shortest $q \subset p$ such that $\beta \leqslant \operatorname{dom} \Phi(q)$. This is the use of the computation $\Phi(p ; \beta)$. We sometimes confuse $\phi(p ; \beta)$ with its domain. We write $\Phi(p ; \beta) \downarrow$ if $\beta \leqslant \operatorname{dom} \Phi(p)$.

We have: if $\gamma<\delta$ and $\Phi(p ; \delta) \downarrow$ then $\phi(p ; \gamma) \leqslant \phi(p ; \delta)$. If $\delta$ is a limit ordinal then $\phi(p ; \delta)=\sup _{\gamma<\delta} \phi(p ; \gamma)$. For all $\gamma$ such that $\Phi(p ; \gamma) \downarrow$ we have $\phi(p ; \gamma) \geqslant \gamma$.

For particular values of a computed function we use the notation $\Phi(p, \gamma)=$ $\Phi(p)(\gamma)$; here $\Phi$ can be either a strong or a weak functional. For a weak functional $\Phi$ we write $\Phi(p, \gamma) \downarrow$ if $\gamma \in \operatorname{dom} \Phi(p)$. We also define the use in an analogous way: $\phi(p, \gamma)$ is the least string $q \subset p$ such that $\Phi(q, \gamma) \downarrow$.

\section{Recursive Approximation}

Let $A$ be an r.e. set and let $A[s]$ be its canonical recursive enumeration. When we take $A[s]$ as an oracle we regard it as a string of length $s$, and let $\Phi(A)[s]=\Phi(A[s])$ (note that this conflicts with our previous definition of $\Phi(p)[s]$, where the object enumerated was $\Phi$ rather than the oracle. Since we will use nice functionals, which are recursive, we will have no use for the original notation.) 
Assume that $A$ is amenable. By admissibility of $\alpha$, we have that for every $\beta<\alpha, A \uparrow \beta \subset A[t]$ from some stage $s<\alpha$ onwards. Thus if $x \in \Phi(A)$ then from some $s$ onwards, $x \in \Phi(A)[t]$. However it is of course possible that for some $x$ and $s$ we have $x \in \Phi(A)[s]$ but $x \notin \Phi(A) ; x$ is enumerated at stage $s$ by a computation that does not agree with $A$, which is later discarded. In fact if $A$ is not low then this may occur for some $x$ at unboundedly many stages $s$.

\section{Miscellany}

Theorem A.12 (Sacks). If $\mathbf{a}$ is an r.e. degree then there is some amenable, r.e. $A \in \mathbf{a}$.

Proof. See [Sac90].

Lemma A.13. If $A \leqslant{ }_{\alpha} B$ and $C$ is r.e. in $B$ then $C$ is r.e. in $A$.

As in classical recursion theory, for $A, B \subset \alpha$ we say that $B \leqslant_{m \alpha} A$ if there is some recursive function $f$ such that $f^{-1} B=A$.

Lemma A.14. Suppose that $B$ is r.e. in $A$ and that $C \leqslant_{m \alpha} B$. Then $C$ is r.e. in $A$.

Proof. Suppose that $f$ is recursive and that $f^{-1} B=C$. If $B=\Phi(A)$ then $C=$ $\Psi(A)$ where

$$
\Psi=\{(p, x):(p, f(x)) \in \Phi\}
$$

\section{Recursive Cofinality}

Let $\Gamma$ be a class of functions. We let

$$
\operatorname{cf}_{\Gamma}(\alpha)=\min \{\gamma: \exists f \in \Gamma(f: \gamma \rightarrow \alpha \text { is cofinal })\}
$$


For example, $\Gamma$ may consist of the functions which are $\Sigma_{n}\left(J_{\alpha}\right)$ or more generally, $\Sigma_{n}\left(J_{\alpha}, A\right)$ for some amenable $A \subset \alpha$. $\alpha$ being admissible means $\operatorname{cf}_{\Sigma_{1}\left(J_{\alpha}\right)}(\alpha)=\alpha$.

Even if $A$ is not amenable then we can let $\Gamma$ be the class of all functions which are weakly recursive in $A$. In this case we write $\operatorname{rcf}(A)$ instead of $\operatorname{cf}_{\Gamma}(\alpha)$. Note that $\operatorname{rcf}\left(0^{\prime}\right)=\operatorname{cf}_{\Sigma_{2}\left(J_{\alpha}\right)}(\alpha)$. 


\section{APPENDIX B}

\section{THE JUMP}

In what follows we confuse functionals and r.e. sets with their indexes. For any amenable $A$ we let

$$
A^{\prime}=\{(\Phi, x): x \in \Phi(A)\}
$$

where $\Phi$ ranges over an effective list of nice enumeration functionals. $A^{\prime}$ is r.e. in $A$; in fact there is a nice enumeration functional $\Phi_{\text {jump }}$ such that for all amenable $A, \Phi_{\text {jump }}(A)=A^{\prime}$. The usual diagonal argument shows that $A^{\prime} \Varangle_{w \alpha} A$.

\section{Characterizations of the Jump}

Lemma B.1. Let $A$ be amenable. If $f \leqslant \leqslant_{w \alpha} A$ and $B=\lim _{s \rightarrow \alpha} f$ then $B \leqslant_{w \alpha} A^{\prime}$. If $A$ is admissible and $B \leqslant \leqslant_{w \alpha} A^{\prime}$ then for some $f \leqslant{ }_{w \alpha} A, B=\lim _{s \rightarrow \alpha} f$.

Proof. Suppose that $f \leqslant \leqslant_{w \alpha} A, B=\lim f$. Let $\Xi$ be a nice weak functional such that $\Xi(A)=f$.

$$
W=\{(x, s): \exists t>s[f(x, t) \neq f(x, s)]\}
$$

is r.e. in $A$ (since it is $\Sigma_{1}\left(L_{\alpha}, A\right)$ and $A$ is amenable), say $W=\Psi(A)=\left(A^{\prime}\right)^{[\Psi]}$. Also say $\left(A^{\prime}\right)^{[\Lambda]}=A$. Let

$$
\Phi=\left\{(p, x): \begin{array}{l}
p \text { is a partial string \& } \\
\exists s\left[p(\Psi,(x, s))=\text { no \& } \Xi\left(p^{[\Lambda]},(x, s)\right)=\text { yes }\right]
\end{array}\right\} .
$$

Then $\Phi\left(A^{\prime}\right)=B$, so $B$ is r.e. in $A^{\prime}$. Similarly we have $\neg B$ r.e. in $A^{\prime}$ so $B \leqslant \leqslant_{w \alpha} A^{\prime}$.

Suppose now that $A$ is admissible and that $B \leqslant{ }_{w \alpha} A^{\prime}$. By Sacks's theorem relativized to $A$ we get an r.e. $C \equiv_{\alpha} A^{\prime}$ which is amenable. Let $\Psi$ be a nice weak functional such that $\Psi(C)=B$. Let $\langle C[s]\rangle$ be an enumeration of $C$, recursive 
in $A$. Let $f(x, s)=\Psi(C[s], x)$ if the latter converges and no otherwise. By the admissibility of $A$, for all $\beta<\alpha$, eventually $C[s] \uparrow \beta \subset C$ so indeed $\lim f=B$.

Lemma B.2. Assume that $A$ is admissible. Then $B \subset \alpha$ is r.e. in $A^{\prime}$ iff it is $\Sigma_{2}(A)$

Proof. If $B \in \Sigma_{2}(A)$, say $x \in B \leftrightarrow \exists y C(x, y)$ where $C \in \Pi_{1}(A) ; C(x, y) \leftrightarrow$ $(C, x, y) \notin A^{\prime}$. It is now easy to see how to enumerate $B$ with oracle $A^{\prime}$.

Let $B$ be r.e. in $A^{\prime}$. Again by Sacks's theorem we get an r.e. $C \equiv_{\alpha} A^{\prime}$ which is amenable; $B=\Phi(C)$ for some nice enumeration functional $\Phi$. Let $\Psi$ be a nice enumeration functional such that $C=\Psi(A)$. By admissibility of $A$ we have that every $\alpha$-finite $K \subset C$ is enumerated by a finite stage, so we have

$$
x \in B \Leftrightarrow \exists K, L, p\left[\begin{array}{l}
L \cap C=0 \quad \& \quad p \subset A \quad \& \quad \forall x \in K((p, x) \in \Psi \quad \& \\
(L \times\{0\} \cup K \times\{1\}, x) \in \Phi
\end{array}\right] .
$$

$L \cap C=0$ is $\Pi_{1}(A)$ and all other clauses in the matrix are recursive in $A$.

\section{Lowness}

A set $A$ is weakly low if $A^{\prime} \leqslant{ }_{w \alpha} 0^{\prime}$. Suppose that $A$ is r.e. and amenable, and fix an effective enumeration $\langle A[s]\rangle$ of $A$. A nice enumeration functional $\Psi$ is weakly low for $A$ if $\Psi(A)=A^{\prime}$ and in addition, for all $x$, if

$$
\{s<\alpha: x \in \Psi(A)[s]\}
$$

is unbounded in $\alpha$, then $x \in A^{\prime}$.

Lemma B.3. An amenable r.e. set $A$ is weakly low iff there is some functional which is weakly low for $A$. . 
Proof. Suppose that $\Psi$ is weakly low for $A$. Let

$$
W=\{(x, s): \exists t>s: x \in \Psi(A)[t]\}
$$

$\neg A^{\prime}$ is r.e. in $W$ as $x \notin A^{\prime}$ iff for some $s,(x, s) \notin W$. $W$ is r.e., so $W \leqslant{ }_{\alpha} 0^{\prime}$. Thus $\neg A^{\prime}$ is r.e. in $0^{\prime} . A \leqslant{ }_{\alpha} 0^{\prime}$ thus $A^{\prime}$ is r.e. in $0^{\prime}$; so $A^{\prime} \leqslant{ }_{w \alpha} 0^{\prime}$.

Now suppose that $A$ is weakly low; let $f(x)[s]$ be a recursive function whose limit is $A^{\prime}$. Let

$$
\Psi=\left\{(p, x):(p, x) \in \Phi_{\text {jump }} \& f(x)[\operatorname{dom} p]=\text { yes }\right\}
$$

$\Psi(A) \subset A^{\prime}$ and if $x \in A^{\prime}$ and $s>\phi_{\text {jump }}(A, x)$ is such that $f(x)[s]=$ yes then $\left(A\lceil s, x) \in \Psi\right.$, thus $\Psi(A)=A^{\prime}$.

Suppose that $x \notin A^{\prime}$; say that for all $s>s_{0}, f(x)[s]=$ no. Suppose that $A\left[s_{1}\right]\left\lceil s_{0}=A \uparrow s_{0}\right.$. Then for $s>s_{1}, s_{0}$ we cannot have $x \in \Psi(A)[s]$; If $(p, x) \in \Psi$ and $p \subset A[s]$ then since $p \not \subset A$ we must have $\operatorname{dom} p>s_{0}$ and so $f(x)[\operatorname{dom} p]=$ no, contradiction.

We say that a set $A$ is low if $A^{\prime} \leqslant_{\alpha} 0^{\prime}$.

Lemma B.4. For all A,

$$
\left\{K \in J_{\alpha}: K \subset \neg A^{\prime}\right\}
$$

is many-one reducible to $\neg A^{\prime}$.

Thus for all $B$, if $\neg A^{\prime}$ is r.e. in $B$ then it is also strongly r.e. in $B$.

Proof. The reduction is the (recursive) function $f$ such that for all $K, f(K)$ is the functional which performs all computations $\Phi(A, x)$ for $(\Phi, x) \in K$ simultaneously and halts if one of these computations halt. Namely:

$$
f(K)=\{p: \exists(\Phi, x) \in K[x \in \Phi(p)]\}
$$


Corollary B.5. Suppose that $A$ is admissible and that $B \geqslant_{\alpha} A$. Then $A^{\prime} \leqslant_{\alpha} B$ iff $A^{\prime} \leqslant w \alpha$.

Proof. $A^{\prime}$ is r.e. in $A$, thus strongly r.e. in $A$ (lemma A.4), thus strongly r.e. in $B$. $\neg A^{\prime}$ is r.e. in $B$ hence strongly r.e. in $B$ (lemma B.4).

Again suppose that $A$ is r.e. and amenable, and fix an effective enumeration $\langle A[s]\rangle$ of $A$. A nice enumeration functional $\Psi$ is low for $A$ if it is weakly low for $A$, and further, for all $\alpha$-finite $K \subset A^{\prime}$, eventually $K \subset \Psi(A)[s]$.

Lemma B.6. If $A$ is r.e. and amenable and there is some functional which is low for $A$ then $A$ is low.

Proof. Suppose that $\Psi$ is low for $A$. Then we already know that $A^{\prime} \leqslant w \alpha 0^{\prime}$, so again we know that $\neg A^{\prime}$ is strongly r.e. in $0^{\prime}$; we need to show that $A^{\prime}$ is strongly r.e. in $0^{\prime}$. But it is strongly r.e. in

$$
W=\{(K, s): \exists t>s: K \not \subset \Psi(A)[t]\}
$$

which is r.e.

Lemma B.7. If $A$ is low, r.e. and admissible, then any functional which is weakly low for $A$ is low for $A$.

The circle is closed via

Lemma B.8. If $A$ is a low r.e. set then $A$ is admissible.

Proof. By Shore [Sho76b], if $\operatorname{rcf}(A)<\alpha$ then $0^{\prime \prime} \leqslant_{\alpha} A^{\prime}$, so $A^{\prime} \leqslant_{\alpha} 0^{\prime}$ is impossible. Also, if $A$ is r.e. and $\operatorname{rcf}(A)=\alpha$ then $A$ is amenable so $A$ is admissible.

Thus an amenable r.e. set $A$ is low iff some functional is low for $A$. 


\section{Uniform Lowness}

A sequence of sets $\left\langle A_{i}\right\rangle_{i \in I}$ is uniformly low if there is a recursive sequence $\left\langle\Phi_{i}\right\rangle_{i \in I}$ such that for all $i, \Phi_{i}\left(0^{\prime}\right)=A_{i}^{\prime}$. Any finite collection of low sets is uniformly low. If $\left\langle A_{i}\right\rangle$ are uniformly low and $\Psi$ is an enumeration functional then

$$
\left\{(i, x): x \in \Psi\left(A_{i}\right)\right\}
$$

is weakly recursive in $0^{\prime}$. 


\section{APPENDIX C}

\section{THE PROJECTUM}

Let $M=\left(J_{\beta}, X\right)$ be amenable. We define $\varrho_{M}$ (also denoted $\left.\varrho_{\beta, X}\right)$ to be the least ordinal $\gamma$ such that

$$
\Sigma_{1}(M) \cap \mathcal{P}(\omega \gamma) \not \subset J_{\beta}
$$

$\varrho_{M}$ is the $\Sigma_{1}$-projectum of $M$. We write $\varrho_{\beta}$ for $\varrho_{\beta, 0}$. $\varrho_{\beta}$ is also denoted $\beta^{*}$.

Claim C.1. If $1<\varrho_{M}<\beta$ then $\varrho_{M}=\omega \varrho_{M}$.

Proof. If $\varrho_{M}<\beta$ then $M \models " \omega \varrho_{M}$ is a cardinal", and so is closed under the function $\gamma \rightarrow \omega \gamma$.

If $\varrho_{M}=1$ then we often confuse 1 and $\omega$ in this context (so we may write $\varrho_{M}=\omega$, meaning the same thing).

Remark C.2. For all $M, \varrho_{M}$ is admissible.

Definition. We say that $M$ is 1 -sound if there is some partial $M$-recursive function $f: \varrho_{M} \rightarrow M$

Lemma C.3. For every $\beta, J_{\beta}$ is 1-sound.

(This is easy if $\beta$ is admissible).

Proof. Let $p$ be the least (in $<_{L}$, or in any canonical well-ordering of finite sets of ordinals) finite set of ordinals such that some $A \subset \varrho_{\beta}$ is $\Sigma_{1}\left(J_{\beta}\right)$ definable with parameters from $\varrho_{\beta} \cup p$ but is not an element of $J_{\beta}$. Let $h$ be the canonical $\Sigma_{1^{-}}$ Skolem function computed in $J_{\beta}$. Let $X=h^{“} \varrho_{\beta} \cup\{p\}$, and let $\pi: X \rightarrow J_{\gamma}$ be the collapse. $A=\pi$ " $A$ is $\Sigma_{1}\left(J_{\gamma}\right)$ so $\gamma=\beta$ (or else $A \in J_{\beta}$ ). Also, it is $\Sigma_{1}$-definable in $J_{\beta}$ from $\varrho_{\beta} \cup \pi(p)$ so by minimality $\pi(p)=p$. It follows that $\pi=\mathrm{id}$. So $h(-, p)$ is the desired function. 
Suppose that $M$ is 1 -sound, and fix a canonical $f: \varrho_{M} \rightarrow M$, say the least one (in $<_{L}$ ). We let $A_{M}=f^{-1} B$ where $B$ is the canonical $\Sigma_{1}(M)$-complete set. We let $M^{\prime}=\left(J_{\varrho_{M}}, A_{M}\right)$.

If in turn $M^{\prime}$ is amenable and 1 -sound then we say that $M$ is 2 -sound and we can proceed to define $M^{\prime \prime}$, etc.

Lemma C.4. (see [Cho84],[Jen72] or [Dod82]) For all $\beta>0$ and $n<\omega, J_{\beta}$ is $n$-sound.

The idea is to imitate the proof of 1 -soundness above, lifting $\pi: J_{\gamma} \rightarrow\left(J_{\beta}\right)^{(n-1)}$ to a $\Sigma_{n}$-elementary map $\bar{\pi}: J_{\delta} \rightarrow J_{\beta}$. We also use

Fact. For all $B \subset \varrho_{M}, B \in \Sigma_{1}\left(M^{\prime}\right)$ iff $B \in \Sigma_{2}(M)$.

Lemma B.2 gives the proof when $M$ is admissible. It is, however, possible for $M$ to be admissible and for $M^{\prime}$ not to be admissible.

For $M$ which is $(n-1)$-sound, we let $\varrho_{M}^{n}=\varrho_{M^{(n-1)}}$. The above facts show that $\varrho_{M}^{n}$ is the least $\gamma$ such that $\Sigma_{n}(M) \cap \mathcal{P}(\omega \gamma) \not \subset M$, the greatest $\gamma$ such that for all $B \in \Sigma_{n}(M) \cap \mathcal{P}(\omega \gamma),\left(J_{\varrho_{M}^{n}}, B\right)$ is amenable and that there is a partial function $p_{n}: \omega \varrho_{M}^{n} \rightarrow M$ which is $\Sigma_{n}(M)$. Combining with the canonical $\Sigma_{1}-$ Skolem function, we get

Corollary C.5 (Jensen's uniformization theorem). For all $\beta$, every $\Sigma_{n}\left(J_{\beta}\right)$ relation can be uniformized by a $\Sigma_{n}\left(J_{\beta}\right)$ function. 


\section{APPENDIX D}

\section{THE ADMISSIBLE COLLAPSE}

In this appendix we review in a unified setting various results of Maass's ([Maa78]) and Sy Friedman's ([Fri82]) regarding isomorphisms between cones of Turing degrees and $\alpha$-degrees. All references will refer to these two papers.

Definition. An amenable $B \subset \alpha$ is collapsible if there is some bijection $p: \omega \leftrightarrow \alpha$, weakly recursive in $B$. A collapse of such a degree is some $\mathcal{B} \subset \omega$ such that for all $A \subset \omega, A$ is $\omega$-r.e. in $\mathcal{B}$ iff it is $\alpha$-r.e. in $B$.

First, we justify the names:

Theorem D.1 (Maass). Every collapsible $\alpha$-degree with an amenable element has a collapse.

Proof. Let $B$ be amenable and let $p: \omega \leftrightarrow \alpha$ be a bijection, weakly recursive in $B$. Let

$$
\mathcal{B}=\{(n, e, s): p(n) \in \Phi(B)[p(s)], \text { where } \Phi=p(e)\}
$$

$\mathcal{B}$ codes the canonical enumerations of all sets r.e. in $B$.

If $A$ is $\omega$-r.e. in $\mathcal{B}$, then $B$ can enumerate it $\alpha$-recursively, because $B$ can $\alpha$ enumerate all finite substrings of $\mathcal{B}$.

In the other direction, note that for every $C \subset \alpha$ which is $\alpha$-r.e. in $B, p^{-1} C$ is $\omega$-r.e. in $\mathcal{B}$. If $A \subset \omega$ is $\alpha$-r.e. in $B$ then $C=p^{\prime \prime} A$ is also $\alpha$-r.e. in $B$ which implies that $A=p^{-1} C$ is $\omega$-r.e. in $\mathcal{B}$.

Next we show some criteria sufficient for the existence of collapsible $\alpha$-degrees. An amenable structure $M=\left(J_{\beta}, X\right)$ is weakly admissible if $\mathrm{cf}_{\Sigma_{1}(M)}(\omega \beta) \geqslant \varrho_{M}$. 
Lemma D.2. Suppose that $\alpha$ is admissible and that $M=\left(J_{\alpha}, A\right)$ is weakly admissible. Then there is a bijection $f: \mathrm{cf}_{\Sigma_{1}(M)} \leftrightarrow \alpha$ which is $\Sigma_{1}(M)$.

Proof. See [Sac90, IX 2.6].

Theorem D.3 (Shore). If $A$ is amenable, r.e. and incomplete (i.e. $\left.0^{\prime} \Varangle_{\alpha} A\right)$ then

$$
\operatorname{rcf}(A) \geqslant \varrho_{\alpha, A}
$$

that is, $\left(J_{\alpha}, \in, A\right)$ is weakly admissible.

Proof. See [Sho76b].

Thus every amenable, incomplete r.e. set with recursive cofinality $\omega$ is collapsible.

Let $B$ be collapsible, and let $\mathcal{B}$ be a collapse of $B$.

Theorem D.4 (Friedmann). Every $\left(J_{\alpha}, B\right)$-degree contains an amenable set.

In fact, since $B$ is amenable, it follows that every $\alpha$-degree above $B$ contains an amenable set. Thus every $\alpha$-degree above $B$ contains a collapsible set.

Lemma D.5. The collapse is a well-defined embedding (preserving $\nless$ ) of the $\left(J_{\alpha}, B\right)$-degrees into the $\left(V_{\omega}, \mathcal{B}\right)$-degrees.

Proof. Suppose that $A, C \geqslant_{\alpha} B$ are amenable and let $\mathcal{A}, \mathcal{C}$ be any collapses of $A, C$ respectively.

Suppose that $A \leqslant_{\alpha} C$. $\mathcal{A}$ and $\neg \mathcal{A}$ are $\omega$-r.e. in $\mathcal{A}$, hence are $\alpha$-r.e. in $A$, hence are $\alpha$-r.e. in $C$, hence are $\omega$-r.e. in $\mathcal{C}$, so $\mathcal{A} \leqslant_{T} \mathcal{C}$.

Suppose that $\mathcal{A} \leqslant_{T} \mathcal{C}$. Let $p: \omega \leftrightarrow \alpha$ be a bijection, weakly recursive in $B$. Consider $\hat{A}=\left\{K \in L_{\alpha}: K \subset A\right\}$ and $\widehat{\neg A}=\left\{K \in L_{\alpha}: K \subset \neg A\right\}$. Both sets are $\alpha$-r.e. in $A$, hence $p^{-1} \hat{A}$ and $p^{-1} \widehat{\neg A}$ are $\alpha$-r.e. in $A$, hence are $\omega$-r.e. in $\mathcal{A}$, hence are $\omega$-r.e. in $\mathcal{C}$, hence are $\alpha$-r.e. in $C$, and so $\hat{A}$ and $\widehat{\neg A}$ are $\alpha$-r.e. in $C$, so $A \leqslant \leqslant_{\alpha} C$. 
In fact, we want to show that the collapse map is an isomorphism.

Definition. A set $C \subset \omega$ is immune if every $\alpha$-finite partial string $q \subset C$ with $\operatorname{dom} q \subset \omega$ is finite.

Lemma D.6. The following are equivalent for immune $A, C \subset \omega$ :

- $A \leqslant_{T} C \oplus \mathcal{B}$.

- $A \leqslant{ }_{w \alpha} C \oplus B$.

- $A \leqslant_{\alpha} C \oplus B$.

Proof. We are asking whether an enumeration of finite (= $\alpha$-finite) substrings of $A$ can be done with oracle $C$ by a functional recursive in $B$, or in $\mathcal{B}$. Since we can restrict ourselves to $\omega$, this is the same question.

Theorem D.7 (Maass, Friedman). Every $\left(J_{\alpha}, B\right)$-degree contains an immune set, and every $\left(V_{\omega}, \mathcal{B}\right)$-degree contains an immune set.

It follows that taking a $B$-degree of an immune set $A$ to the $\mathcal{B}$-degree of $A$ is well-defined and is an isomorphism between the $B$ degrees and the $\mathcal{B}$ degrees. We want to show that this map is the same as the admissible collapse. We need to show

Lemma D.8. Let $A \geqslant_{\alpha} B$ be amenable, let $\mathcal{A}$ be its collapse, and let $E$ be an immune set such that $E \equiv_{B} A$. Then $E \equiv_{\mathcal{B}} \mathcal{A}$.

Proof. $\mathcal{A} \leqslant_{w \alpha} A$, and so $\mathcal{A} \leqslant_{w B} E$. This implies that there is some weak $\left(J_{\alpha}, B\right)$ functional $\Phi$ such that $\Phi(E)=\mathcal{A}$. Consider $\Phi_{0}=\Phi \cap V_{\omega}$. $\Phi_{0}$ is still $\alpha$-r.e. in $B$, and $\Phi_{0}(E)=\mathcal{A}$ because $E$ is immune. $\Phi_{0}$ is $\omega$-r.e. in $\mathcal{B}$, so $\mathcal{A} \leqslant_{T} \mathcal{B} \oplus E$, in other words, $\mathcal{A} \leqslant_{\mathcal{B}} E$.

On the other hand, $E \leqslant_{w \alpha} A$, and so $E \leqslant_{T} \mathcal{A}$. 
Let $j$ be the inverse of the collapse map, from the Turing degrees above $\mathcal{B}$ to the $\alpha$-degrees above $B$ (equivalently, from the $\mathcal{B}$-degrees to the $B$-degrees).

\section{The Collapse and Recursively Enumerable Degrees}

Lemma D.9 (Maass, Friedman). A B -degree $\mathbf{d}$ contains a set $\omega$-r.e. in $\mathcal{B}$ iff $j(\mathbf{d})$ contains a set which is strongly $\alpha-r$.e. in $B$.

Corollary D.10. For every $\mathcal{B}$-degrees $\mathbf{d}_{1} \leqslant \mathbf{d}_{2}, \mathbf{d}_{2}$ contains a set $\omega$-r.e. in $\mathbf{d}_{1}$ iff $j\left(\mathbf{d}_{2}\right)$ contains a set strongly $\alpha-r . e$. in $j\left(\mathbf{d}_{1}\right)$.

Proof. Lemma D.9 implies that a Turing degree $\mathbf{d}$ above $\mathcal{B}$ contains a set $\omega$-r.e. in $\mathcal{B}$ iff $j(\mathbf{d})$ contains a set $\alpha$-r.e. in $B$. Now relativize this lemma, using $\mathbf{d}_{1}$ as the base of the cone.

Theorem D.11 (Maass). An $\alpha$-degree $\mathbf{d}$ above $B$ is r.e. in $B$ iff $j^{-1}(\mathbf{d})$ is r.e. and above a degree $\mathbf{a}$ which is r.e. and above $\mathcal{B}$.

As a corollary, we have that for every Turing degree $\mathbf{d}$ above $\mathcal{B}, j\left(\mathbf{d}^{\prime \prime}\right)=j(\mathbf{d})^{\prime}$. 


\section{APPENDIX E}

\section{PROMPT PERMISSION}

In this appendix we develop some of the theory of promptly simple sets in the context of admissible recursion theory. In fact, in the context of the r.e. degrees (rather than the lattice of sets), the basic notion seems to be that of prompt permitting.

Definition. An amenable r.e. set A permits promptly if there is some effective enumeration $\langle A[s]\rangle$ of $A$ and a recursive function $p$ such that for all $s, p(s) \geqslant s$, and for all $e$ such that $W_{e}$ is unbounded in $\alpha$, there is some $x \in W_{e}$ which enters $W_{e}$ at some $s$ (according to the canonical enumeration of the $W_{e} \mathrm{~s}$ ) and such that

$$
A \uparrow x+1[s] \neq A \uparrow x+1[p(s)] .
$$

Lemma E.1 (Uniform Slowdown Lemma). There is a recursive function $f$ such that for all $\epsilon$, if $\epsilon$ is a recursive index for an array of enumerations $\left\langle U_{e}[s]\right\rangle$ of r.e. sets $U_{e}$, then $f(\epsilon)$ is a recursive index for a total recursive function $g$ such that for all e, $W_{g(e)}=U_{e}$ and every number in $U_{e}$ enters $U_{e}$ (according to the given approximation) before it enters $W_{g(e)}$ (according to the canonical enumeration).

Proof. We observe that the classical proof of the slowdown lemma (see [Soa87, XIII 1.5]) is easily generalized to the admissible context and is uniform. For any recursive index $\epsilon$, let

$$
U_{e}^{\epsilon}[s]=\cup\left\{\varphi_{\epsilon}(e, x): x<s, \varphi_{\epsilon}(e, x) \downarrow[s]\right\} .
$$

Find some recursive function $h$ such that for all $\epsilon, e, i$,

$$
W_{h(\epsilon, e, i)}=\left\{x: \exists s\left(x \in U_{e}^{\epsilon}[s] \backslash W_{i}[s]\right)\right\} .
$$


By the recursion theorem with parameters, find some recursive function $k$ such that for all $e$ and $\epsilon, W_{k(\epsilon, e)}=W_{h(\epsilon, e, k(\epsilon, e))}$. Let $f(\epsilon)$ be the index for $k(\epsilon,-)$.

Lemma E.2. Suppose that $\left\langle U_{e}[s]\right\rangle$ is an effective enumeration of an array of r.e. sets $U_{e}$ such that there is a recursive bound for $\cup\left\{U_{e}[s]: e \leqslant s\right\}$, and suppose that A permits promptly. Then there is some recursive function $q$ such that for all $s, q(s) \geqslant s$ and such that for all $e$, if $U_{e}$ is unbounded then there is some $x \in U_{e}$ which enters $U_{e}$ at $s$ and such that

$$
A \uparrow x+1[s] \neq A \uparrow x+1[q(s)]
$$

An index for $q$ can be obtained uniformly from an index for $\left\langle U_{e}[s]\right\rangle$.

Proof. Suppose that $p$ and $\langle a[s]\rangle$ witness that $A$ permits promptly. Find a recursive $g$ such that for all $e, W_{g(e)}=U_{e}$ and the former is enumerated more slowly. Define

$$
q(s)=\sup \left\{p(t): \exists x, e\left(x \text { enters } U_{e} \text { at } s \quad \& \quad x \text { enters } W_{g(e)} \text { at } t\right)\right\}
$$

An index for $q$ can be obtained from an index for $g$.

The last lemma justifies the technique used in chapter 2 to use prompt permission. In the verifications of that chapter, we use a variety of r.e. sets constructed during the construction and argue that during the construction, those sets which are unbounded received prompt permission. This is legal because by the recursion theorem, we could use a permitting function $p$ which permits with respect to the sets being constructed. 


\section{BIBLIOGRAPHY}

[Cho84] C. T. Chong. Techniques of admissible recursion theory, volume 1106 of Lecture Notes in Mathematics. Springer-Verlag, Berlin, 1984.

[Chu38] Alonzo Church. The constructive second number class. Bull. Amer. Math. Soc., 44:224-232, 1938.

[CK37] Alonzo Church and Stephen Cole Kleene. Formal definitions in the theory of ordinal numbers. Fund. Math., 28:11-21, 1937.

[Dod82] A. J. Dodd. The core model, volume 61 of London Mathematical Society Lecture Note Series. Cambridge University Press, Cambridge, 1982.

[Dri68] Graham C. Driscoll, Jr. Metarecursively enumerable sets and their metadegrees. J. Symbolic Logic, 33:389-411, 1968.

[DS96] Rod Downey and Richard A. Shore. Lattice embeddings below a nonlow 2 recursively enumerable degree. Israel J. Math., 94:221-246, 1996.

[Fej82] Peter A. Fejer. Branching degrees above low degrees. Trans. Amer. Math. Soc., 273(1):157-180, 1982.

[Fri82] Sy D. Friedman. The Turing degrees and the metadegrees have isomorphic cones. In Patras Logic Symposion (Patras, 1980), volume 109 of Stud. Logic Foundations Math., pages 145-157. North-Holland, Amsterdam, 1982.

[G3̈9] Kurt Friedrich Gödel. Consistency-proof for the generalized continuumhypothsis. Proc. Nat. Acad. Sci. USA, 25:220-224, 1939.

[GSS] Noam Greenberg, Richard A. Shore, and Theodore A. Slaman. The theory of the metarecursively enumerable degrees. Submitted.

[HS82] Leo Harrington and Saharon Shelah. The undecidability of the recursively enumerable degrees. Bull. Amer. Math. Soc. (N.S.), 6(1):79-80, 1982.

[Jec03] Thomas Jech. Set theory. Springer Monographs in Mathematics. Springer-Verlag, Berlin, 2003. The third millennium edition, revised and expanded.

[Jen72] R. Björn Jensen. The fine structure of the constructible hierarchy. Ann. Math. Logic, 4:229-308; erratum, ibid. 4 (1972), 443, 1972. With a section by Jack Silver.

[Kle38] Stephen Cole Kleene. On notations for ordinal numbers. J. Symbolic Logic, 3:150-155, 1938. 
[Kre61] G. Kreisel. Set theoretic problems suggested by the notion of potential totality. In Infinitistic Methods (Proc. Sympos. Foundations of Math., Warsaw, 1959), pages 103-140. Pergamon, Oxford, 1961.

[Kri64] Saul Kripke. Transfinite recursion on admissible ordinals i, ii (abstracts). J. Symbolic Logic, 29:161-162, 1964.

[KS63] G. Kreisel and Gerald E. Sacks. Metarecursive sets i, ii (abstracts). J. Symbolic Logic, 28:304-305, 1963.

[KS65] G. Kreisel and Gerald E. Sacks. Metarecursive sets. J. Symbolic Logic, 30:318-338, 1965.

[Lac72] A. H. Lachlan. Embedding nondistributive lattices in the recursively enumerable degrees. In Conference in Mathematical Logic-London '70 (Proc. Conf., Bedford Coll., London, 1970), pages 149-177. Lecture Notes in Math., Vol. 255. Springer, Berlin, 1972.

[Lac76] Alistair H. Lachlan. A recursively enumerable degree which will not split over all lesser ones. Ann. Math. Logic, 9(4):307-365, 1976.

[Lac79] A. H. Lachlan. Bounding minimal pairs. J. Symbolic Logic, 44(4):626$642,1979$.

[Ler74] Manuel Lerman. Maximal $\alpha$-r.e. sets. Trans. Amer. Math. Soc., 188:341386, 1974.

[LS72] Manuel Lerman and Gerald E. Sacks. Some minimal pairs of $\alpha$ recursively enumerable degrees. Ann. Math. Logic, 4:415-442, 1972.

[LS73] M. Lerman and S. G. Simpson. Maximal sets in $\alpha$-recursion theory. Israel J. Math., 14:236-247, 1973.

[Maa78] Wolfgang Maass. Inadmissibility, tame r.e. sets and the admissible collapse. Ann. Math. Logic, 13(2):149-170, 1978.

[Myt89] Michael Mytilinaios. Finite injury and $\Sigma_{1}$-induction. J. Symbolic Logic, 54(1):38-49, 1989.

[NSS98] André Nies, Richard A. Shore, and Theodore A. Slaman. Interpretability and definability in the recursively enumerable degrees. Proc. London Math. Soc. (3), 77(2):241-291, 1998.

[Ode83] David Odell. Trace constructions in $\alpha$-recursion theory. PhD thesis, Cornell University, Ithaca, NY, 1983.

[Pla65] Richard Platek. Foundations of recursion theory. PhD thesis, Stanford Univeristy, Stanford, CA, 1965. 
[Sac63] Gerald E. Sacks. Degrees of unsolvability. Princeton University Press, Princeton, N.J., 1963.

[Sac66] Gerald E. Sacks. Metarecursively enumerable sets and admissible ordinals. Bull. Amer. Math. Soc., 72:59-64, 1966.

[Sac90] Gerald E. Sacks. Higher recursion theory. Perspectives in Mathematical Logic. Springer-Verlag, Berlin, 1990.

[Sho76a] Richard A. Shore. On the jump of an $\alpha$-recursively enumerable set. Trans. Amer. Math. Soc., 217:351-363, 1976.

[Sho76b] Richard A. Shore. The recursively enumerable $\alpha$-degrees are dense. Ann. Math. Logic, 9(1-2):123-155, 1976.

[Sho78] Richard A. Shore. On the $\forall \exists$-sentences of $\alpha$-recursion theory. In Generalized recursion theory, II (Proc. Second Sympos., Univ. Oslo, Oslo, 1977), volume 94 of Stud. Logic Foundations Math., pages 331-353. North-Holland, Amsterdam, 1978.

[Sho82] Richard A. Shore. On homogeneity and definability in the first-order theory of the Turing degrees. J. Symbolic Logic, 47(1):8-16, 1982.

[Sho97] Richard A. Shore. Conjectures and questions from Gerald Sacks's degrees of unsolvability. Arch. Math. Logic, 36(4-5):233-253, 1997. Sacks Symposium (Cambridge, MA, 1993).

[Sho99] Richard A. Shore. The recursively enumerable degrees. In Handbook of computability theory, volume 140 of Stud. Logic Found. Math., pages 169-197. North-Holland, Amsterdam, 1999.

[Sla91] Theodore A. Slaman. Degree structures. In Proceedings of the International Congress of Mathematicians, Vol. I, II (Kyoto, 1990), pages 303-316, Tokyo, 1991. Math. Soc. Japan.

[Soa87] Robert I. Soare. Recursively enumerable sets and degrees. Perspectives in Mathematical Logic. Springer-Verlag, Berlin, 1987. A study of computable functions and computably generated sets.

[SS72] G. E. Sacks and S. G. Simpson. The $\alpha$-finite injury method. Ann. Math. Logic, 4:343-367, 1972.

[SS90] Juichi Shinoda and Theodore A. Slaman. On the theory of the PTIME degrees of the recursive sets. J. Comput. System Sci., 41(3):321-366, 1990 .

[Suk69] J. Sukonick. Lower bounds for pairs of metarecursively enumerable degrees. PhD thesis, M.I.T., Cambridge, MA, 1969. 
[SW89] Theodore A. Slaman and W. Hugh Woodin. $\Sigma_{1}$-collection and the finite injury priority method. In Mathematical logic and applications (Kyoto, 1987), volume 1388 of Lecture Notes in Math., pages 178-188. Springer, Berlin, 1989.

[Tak60] Gaisi Takeuti. On the recursive functions of ordinal numbers. J. Math. Soc. Japan, 12:119-128, 1960.

[Tak65] Gaisi Takeuti. A formalization of the theory of ordinal numbers. $J$. Symbolic Logic, 30:295-317, 1965.

[Wei88] B. Weinstein. On embeddings of the 1-3-1 lattice into the recursively enumerable degrees. PhD thesis, University of California, Berkeley, 1988. 